hep-ph/0112113

CERN-TH/2001-339

UMN-TH-2032/01

TPI-MINN-01/50

\title{
Calculations of Neutralino-Stop Coannihilation in the CMSSM
}

\author{
John Ellis ${ }^{1}$, Keith A. Olive ${ }^{2}$ and Yudi Santoso ${ }^{2}$ \\ ${ }^{1}$ TH Division, CERN, Geneva, Switzerland \\ ${ }^{2}$ Theoretical Physics Institute, University of Minnesota, Minneapolis, MN 55455, USA
}

\begin{abstract}
We present detailed calculations of the $\tilde{\chi} \tilde{t}_{1}$ coannihilation channels that have the largest impact on the relic $\tilde{\chi}$ density in the constrained minimal supersymmetric extension of the Standard Model (CMSSM), in which scalar masses $m_{0}$, gaugino masses $m_{1 / 2}$ and the trilinear soft supersymmetry-breaking parameters $A_{0}$ are each assumed to be universal at some input grand unification scale. The most important $\tilde{t}_{1} \tilde{t}_{1}^{*}$ and $\tilde{t}_{1} \tilde{t}_{1}$ annihilation channels are also calculated, as well as $\tilde{t}_{1} \tilde{\ell}$ coannihilation channels. We illustrate the importance of these new coannihilation calculations when $A_{0}$ is relatively large. While they do not increase the range of $m_{1 / 2}$ and hence $m_{\chi}$ allowed by cosmology, these coannihilation channels do open up new 'tails' of parameter space extending to larger values of $m_{0}$.
\end{abstract}

CERN-TH/2001-339

November 2001 


\section{Introduction}

A favoured candidate for cold dark matter is the lightest supersymmetric particle (LSP), which is generally thought to be the lightest neutralino $\tilde{\chi}$ [1] in the minimal supersymmetric extension of the Standard Model (MSSM). It is common to focus attention on the constrained MSSM (CMSSM), in which all the soft supersymmetry-breaking scalar masses $m_{0}$ are required to be equal at an input superysmmetric GUT scale, as are the gaugino masses $m_{1 / 2}$ and the trilinear soft supersymmetry-breaking parameters $A_{0}$. These assumptions yield well-defined relations between the various sparticle masses, and correspondingly more definite predictions for the relic abundance $\Omega_{\tilde{\chi}} h^{2}$ and observable signatures. This paper is devoted to relic-abundance calculations including coannihilations of the lightest neutralino $\tilde{\chi}$ with $\tilde{t}_{1}$, the lighter supersymmetric partner of the top quark [2].

The range $0.1<\Omega_{\tilde{\chi}} h^{2}<0.3$ is generally thought to be preferred by astrophysics and cosmology [3]. Lower values of $\Omega_{\tilde{\chi}} h^{2}$ might be possible if there is some other source of cold dark matter, but higher values are incompatible with observation. The regions of the $m_{1 / 2}, m_{0}$ plane where the relic density falls within the preferred range $0.1<\Omega_{\tilde{\chi}} h^{2}<0.3$ have generally been divided into four generic parts. There is a 'bulk' region at moderate $m_{1 / 2}$ and $m_{0}$ [1]. Then, extending to larger $m_{1 / 2}$, there is a 'tail' of the parameter space where the LSP $\tilde{\chi}$ is almost degenerate with the next-to-lightest supersymmetric particle (NLSP), which is in this region the $\tilde{\tau}_{1}$, the lighter supersymmetric partner of the $\tau$ lepton. Along this 'tail', efficient coannihilations [4, 5, 6] keep $\Omega_{\widetilde{\chi}} h^{2}$ down in the preferred range, even for larger values of $m_{\tilde{\chi}}[7,8,9,10]$. At larger $m_{0}$, close to the boundary where electroweak symmetry breaking is no longer possible, there is the 'focus-point' region where the LSP has a larger Higgsino component and $m_{\tilde{\chi}}$ is small enough for $\Omega_{\tilde{\chi}} h^{2}$ to be acceptable [11]. Finally, extending to larger $m_{1 / 2}$ and $m_{0}$ at intermediate values of $m_{1 / 2} / m_{0}$, there may be a 'funnel' of CMSSM parameter space where rapid direct-channel annihilations via the poles of the heavier Higgs bosons $A$ and $H$ keep $\Omega_{\widetilde{\chi}} h^{2}$ in the preferred range [12, 13].

In this paper, we emphasize the significance of coannihilation of the LSP $\tilde{\chi}$ with $\tilde{t}_{1}$, the lighter supersymmetric partner of the $t$ quark [2]. This mechanism opens up another 'tail'

of parameter space, this time extending to larger values of $m_{0}$. It is not relevant for the small values of $A_{0}$ considered in previous coannihilation calculations [8, 9, [1], but may be important for large $A_{0}$, as we demonstrate in this paper. Coannihilations of $\tilde{\chi}$ with $\tilde{t}_{1}$ are important when the latter is the NLSP, just as $\tilde{\chi} \tilde{\tau}_{1}$ coannihilations are important when the $\tilde{\tau}_{1}$ is the NLSP. In the latter case, one must also consider coannihilations with the $\tilde{e}_{1}$ and $\tilde{\mu}_{1}$, which are not much heavier than the $\tilde{\tau}_{1}[7,8,8,10]$. There are also regions of 
CMSSM parameter space where both the $\tilde{t}_{1}$ and $\tilde{\tau}_{1}$ are close in mass to the LSP $\tilde{\chi}$, and $\tilde{t}_{1} \tilde{\tau}_{1}$ coannihilations must also be considered. We present here detailed calculations of the matrix elements and cross sections for all the leading $\tilde{\chi} \tilde{t}_{1}$ and $\tilde{t}_{1} \tilde{\ell}$ coannihilation processes, and illustrate their importance for $\Omega_{\tilde{\chi}} h^{2}$ in some instances in the CMSSM when $A_{0} \neq 0$.

The structure of the paper is as follows. In Section 2 we recall some important features of LSP relic-density calculations in general, and coannihilations in particular. Then, in Section 3 we compare the relative magnitudes of the $\tilde{\chi} \tilde{\chi}, \tilde{\chi} \tilde{t}_{1}, \tilde{t}_{1} \tilde{t}_{1}^{(*)}$ and $\tilde{t}_{1} \tilde{\ell}^{(*)}$ processes for some specific choices of the CMSSM parameters. Section 4 provides an overview of the implications of $\tilde{\chi} \tilde{t}_{1}$ coannihilation and related processes for the regions of the $m_{1 / 2}, m_{0}$ plane allowed by the constraint $0.1<\Omega_{\tilde{\chi}} h^{2}<0.3$ for various choices of the other CMSSM parameters. Relevant details of our calculations of the matrix elements are contained in an Appendix.

\section{Formalism for Annihilation and Coannihilation}

The density of neutralino relics left over from the early Universe may be determined relatively simply in terms of relevant annihilation cross sections, using the Boltzmann rate equation to determine a freeze-out density. The relic density subsequently scales with the inverse of the comoving volume, and hence with the entropy density. In the MSSM framework discussed here, since neutralinos are Majorana fermions, the $S$-wave annihilation cross sections into fermion-antifermion pairs are suppressed by the masses of the final-state fermions, and it is therefore necessary to compute $P$-wave contributions to the cross sections [1].

The rate equation for a stable particle with density $n$ is

$$
\frac{d n}{d t}=-3 \frac{\dot{R}}{R} n-\left\langle\sigma v_{\mathrm{rel}}\right\rangle\left(n^{2}-n_{\mathrm{eq}}^{2}\right),
$$

where $n_{\text {eq }}$ is the equilibrium number density and $\left\langle\sigma v_{\text {rel }}\right\rangle$ is the thermally averaged product of the annihilation cross section $\sigma$ and the relative velocity $v_{\text {rel }}$. In the early Universe, we can write $\dot{R} / R=\left(8 \pi G_{N} \rho / 3\right)^{1 / 2}$, where $\rho=\pi^{2} g(T) T^{4} / 30$ is the energy density in radiation and $g(T)$ is the number of relativistic degrees of freedom. Conservation of the entropy density $s=2 \pi^{2} h(T) T^{4} / 45$ implies that $\dot{R} / R=-\dot{T} / T-h^{\prime} \dot{T} / 3 h$ where $h^{\prime} \equiv d h / d T$. Generally, we have $h(T) \approx g(T)$. Defining $x \equiv T / m$ and $q \equiv n / T^{3} h$, we can write

$$
\frac{d q}{d x}=m\left(\frac{4 \pi^{3}}{45} G_{N} g\right)^{-1 / 2}\left(h+\frac{1}{3} m x h^{\prime}\right)\left\langle\sigma v_{\mathrm{rel}}\right\rangle\left(q^{2}-q_{\mathrm{eq}}^{2}\right) .
$$

The effect of the $h^{\prime}$ term was discussed in detail in [14, and is most important when the mass $m$ is between 2 and $10 \mathrm{GeV}$. Since we only consider neutralinos that are significantly 
more massive, we neglect it below (though it is not neglected in our calculations). In the case of the MSSM, freeze-out occurs when $x \sim 1 / 20$, and the final relic density is determined by integrating (1) down to $x=0$, and is given by

$$
\rho_{\tilde{\chi}}=m q(0) h(0) T_{0}^{3} .
$$

When coannihilations are important, there are several relevant particle species $i$, each with different mass, number density $n_{i}$ and equilibrium number density $n_{\mathrm{eq}, i}$. Even in such a situation [4], the rate equation (1) still applies, provided $n$ is interpreted as the total number density,

$$
n \equiv \sum_{i} n_{i}
$$

$n_{\text {eq }}$ as the total equilibrium number density,

$$
n_{\mathrm{eq}} \equiv \sum_{i} n_{\mathrm{eq}, i}
$$

and the effective annihilation cross section as

$$
\left\langle\sigma_{\mathrm{eff}} v_{\mathrm{rel}}\right\rangle \equiv \sum_{i j} \frac{n_{\mathrm{eq}, i} n_{\mathrm{eq}, j}}{n_{\mathrm{eq}}^{2}}\left\langle\sigma_{i j} v_{\mathrm{rel}}\right\rangle
$$

In (21), $m$ is now understood as the mass of the lightest particle under consideration. For $T \lesssim m_{i}$, the equilibrium number density of each species is given by [14, 15

$$
\begin{aligned}
n_{\mathrm{eq}, i} & =g_{i} \int \frac{d^{3} p}{(2 \pi)^{3}} e^{-E / T} \\
& =\frac{g_{i} m_{i}^{2} T}{2 \pi^{2}} K_{2}\left(m_{i} / T\right), \\
& =g_{i}\left(\frac{m_{i} T}{2 \pi}\right)^{3 / 2} \exp \left(-m_{i} / T\right)\left(1+\frac{15 T}{8 m_{i}}+\ldots\right),
\end{aligned}
$$

where $g_{i}$ is a spin and color degeneracy factor and $K_{2}(x)$ is a modified Bessel function. We make the approximation of Boltzmann statistics for the annihilating particles, which is excellent in practice.

We now recall how to compute $\left\langle\sigma_{12} v_{\text {rel }}\right\rangle$ for the process $1+2 \rightarrow 3+4$ in an efficient manner. Suppose we have determined the squared transition matrix element $|\mathcal{T}|^{2}$ (summed over final spins and averaged over initial spins) and expressed it as a function of the Mandelstam variables $s, t, u$. We then express $|\mathcal{T}|^{2}$ in terms of $s$ and the scattering angle $\theta_{\mathrm{CM}}$ in the center-of-mass frame, as described in [8]. We now define

$$
\begin{aligned}
w(s) & \equiv \frac{1}{4} \int \frac{d^{3} p_{3}}{(2 \pi)^{3} E_{3}} \frac{d^{3} p_{4}}{(2 \pi)^{3} E_{4}}(2 \pi)^{4} \delta^{4}\left(p_{1}+p_{2}-p_{3}-p_{4}\right)|\mathcal{T}|^{2} \\
& =\frac{1}{32 \pi} \frac{p_{3}(s)}{s^{1 / 2}} \int_{-1}^{+1} d \cos \theta_{\mathrm{CM}}|\mathcal{T}|^{2} .
\end{aligned}
$$


In terms of $w(s)$, the total annihilation cross section $\sigma_{12}(s)$ is given by $\sigma_{12}(s)=w(s) / s^{1 / 2} p_{1}(s)$ 凹.

The above analysis is exact. To reproduce the usual partial wave expansion, we expand $|\mathcal{T}|^{2}$ in powers of $p_{1}(s) / m_{1}$. The odd powers vanish upon integration over $\theta_{\mathrm{CM}}$, while the zeroth- and second-order terms correspond to the usual $S$ and $P$ waves, respectively. Each factor of $p_{1}(s)$ is accompanied by a factor of $\cos \theta_{\mathrm{CM}}$, so we have

$$
\int_{-1}^{+1} d \cos \theta_{\mathrm{CM}}|\mathcal{T}|^{2}=\left(|\mathcal{T}|_{\cos \theta_{\mathrm{CM}} \rightarrow+1 / \sqrt{3}}^{2}+|\mathcal{T}|_{\cos \theta_{\mathrm{CM}} \rightarrow-1 / \sqrt{3}}^{2}+\mathcal{O}\left(p_{1}^{4}\right)\right.
$$

We can therefore evaluate the $S$ - and $P$-wave contributions to $w(s)$ simply by evaluating $|\mathcal{T}|^{2}$ at two different values of $\cos \theta_{\mathrm{CM}}$; no integrations are required.

The proper procedure for thermal averaging has been discussed in [14, 15] for the case of $m_{1}=m_{2}$, and in [16, 6] for the case of $m_{1} \neq m_{2}$, so we do not discuss it in detail here. One finds

$$
\left\langle\sigma_{12} v_{\text {rel }}\right\rangle=a_{12}+b_{12} x+\mathcal{O}\left(x^{2}\right)
$$

where $x \equiv T / m_{1}$ (assuming $m_{1}<m_{2}$ ). In our case, we extract $a_{12}$ and $b_{12}$ from the transition amplitudes listed in the Appendix for each final state. We set $x=0$ to get $a_{12}$, and then compute $b_{12}$ by setting $x$ to a numerical value small enough to render the $\mathcal{O}\left(x^{2}\right)$ terms negligible. We then compute $a_{\text {eff }}$ and $b_{\text {eff }}$ by performing the sum over initial states as in (6), and integrate the rate equation (2) numerically to obtain the relic LSP abundance. To a fair approximation, the relic density can simply be written as [1], 世]

$$
\Omega h^{2} \approx \frac{10^{9} \mathrm{GeV}^{-1}}{g_{f}^{1 / 2} M_{\mathrm{pl}}\left(a_{\mathrm{eff}}+b_{\mathrm{eff}} x_{f} / 2\right) x_{f}},
$$

where the freeze-out temperature $T_{f} \sim m_{\tilde{\chi}} / 20$, and $g_{f}$ is the number of relativistic degrees of freedom at $T_{f}$.

This implies that the ratio of relic densities computed with and without coannihilations is approximately

$$
R \equiv \frac{\Omega^{0}}{\Omega} \approx\left(\frac{\hat{\sigma}_{\mathrm{eff}}}{\hat{\sigma}_{0}}\right)\left(\frac{x_{f}}{x_{f}^{0}}\right),
$$

where $\hat{\sigma} \equiv a+b x / 2$ and sub- and superscripts 0 denote quantities computed ignoring coannihilations. The ratio $x_{f}^{0} / x_{f} \approx 1+x_{f}^{0} \ln \left(g_{\text {eff }} \sigma_{\text {eff }} / g_{1} \sigma_{0}\right)$, where $g_{\text {eff }} \equiv \sum_{i} g_{i}\left(m_{i} / m_{1}\right)^{3 / 2} e^{-\left(m_{i}-m_{1}\right) / T}$. For the case where the $\tilde{t}_{1}$ and $\tilde{\chi}$ are almost degenerate, $g_{\text {eff }} \approx \sum_{i} g_{i}=8$ and $x_{f}^{0} / x_{f} \approx 1.2$.

\footnotetext{
${ }^{1} \operatorname{Our} w(s)$ is also the same as $w(s)$ in [14, 16, 7], which is written as $W / 4$ in [6].
} 


\section{Coannihilation Rates for $\tilde{t}_{1}$ in the MSSM}

We now use the above formalism to estimate the relative importance of the $\tilde{t}_{1} \tilde{\chi}$ coannihilation processes, $\tilde{t}_{1} \tilde{t}_{1}^{*}$ and $\tilde{t}_{1} \tilde{t}_{1}$ annihilations calculated in the Appendix. We also take into account the $\tilde{\chi} \tilde{\ell}$ coannihilations calculated previously [7, [8] and, for completeness, include the $\tilde{t}_{1} \tilde{\ell}$ and $\tilde{t}_{1} \tilde{\ell}^{*}$ coannihilations also calculated in the Appendix.

To compute the effective annihilation cross sections for light sparticles in the MSSM, we allow the index $i$ in (四) to run over $\tilde{t}_{1}, \tilde{t}_{1}^{*}, \widetilde{\tau}_{1}, \widetilde{\tau}_{1}^{*}, \widetilde{e}_{\mathrm{R}}, \widetilde{e}_{\mathrm{R}}^{*}, \widetilde{\mu}_{\mathrm{R}}$ and $\widetilde{\mu}_{\mathrm{R}}^{*}$, as well as $\tilde{\chi}$. The following is the change in $\sigma_{\text {eff }}$ compared with [8], where 49 of the $\sigma_{i j}$ in (6) were already included:

$$
\Delta \sigma_{\text {eff }}=2\left(\sigma_{\tilde{t}_{1} \tilde{t}_{1}}+\sigma_{\tilde{t}_{1} \tilde{t}_{1}^{*}}\right) r_{\tilde{t}_{1}}^{2}+4 \sigma_{\widetilde{\chi} \tilde{t}_{1}} r_{\widetilde{\chi}} r_{\tilde{t}_{1}}+8\left(\sigma_{\tilde{t}_{1} \tilde{e}_{R}}+\sigma_{\tilde{t}_{1} \tilde{e}_{R}^{*}}\right) r_{\tilde{t}_{1}} r_{\tilde{e}_{R}}+4\left(\sigma_{\tilde{t}_{1} \tilde{\tau}_{1}}+\sigma_{\tilde{t}_{1} \tilde{\tau}_{1}^{*}}\right) r_{\widetilde{\tau}_{1}} r_{\tilde{t}_{1}}
$$

where $r_{i} \equiv n_{\text {eq }, i} / n_{\text {eq }}$. We have taken the $\widetilde{e}_{\mathrm{R}}$ and $\widetilde{\mu}_{\mathrm{R}}$ (but not the $\tilde{\tau}$ ) to be degenerate in mass, thus accounting for the 81 possible initial state combinations. Note that we have summed over color states in the cross sections amplitudes listed in the Appendix, and we have taken the stop degeneracy factor $g_{\tilde{t}}=3$. We list in Table 1 the sets of initial and final states for which we compute the annihilation cross sections, using the transition amplitudes given in the Appendix. We use $q$ to denote the four light quarks, which we have taken to be massless.

Table 1: Initial and Final States for $\tilde{t}_{1}$ Annihilation and Coannihilation Processes

\begin{tabular}{|c|l|}
\hline Initial State & Final States \\
\hline$\tilde{t}_{1} \tilde{t}_{1}^{*}$ & $g g, \gamma g, Z g, t \bar{t}, b \bar{b}, q \bar{q}, g h, g H, Z h, Z H, Z A, W^{ \pm} H^{\mp}$, \\
& $h h, h H, H H, A A, h A, H A, H^{+} H^{-}$ \\
$\tilde{t}_{1} \tilde{t}_{1}$ & $t t$ \\
$\tilde{\chi}_{t_{1}}$ & $t g, t Z, b W^{+}, t H, t h, t A, b H^{+}$ \\
$\tilde{t}_{1} \tilde{\ell}_{1}$ & $t \ell, b \nu$ \\
$\tilde{t}_{1} \tilde{\ell}^{*}$ & $t \bar{\ell}$ \\
\hline
\end{tabular}

In the CMSSM, the diagonal entries of the squark mass matrix tend to pick up large contributions from the gaugino masses, $m_{L L, R R}^{2} \ni \mathcal{O}(6) m_{1 / 2}^{2}$, thus making the squarks heavier than the neutralinos. The off-diagonal entry for an up-type squark $]$

$$
m_{L R}^{2}=-m_{q}\left(A_{q}+\mu \cot \beta\right)
$$

can, however, be large, particularly for the stops, or for sbottoms at large $\tan \beta$. When $A_{t}$ is sufficiently large, the lighter stop, $\tilde{t}_{1}$, can become degenerate with (or lighter than)

\footnotetext{
${ }^{2}$ Note here our sign convention for $A_{q}$.

${ }^{3}$ For down-type squarks, the factor $\cot \beta$ in (14) is replaced by $\tan \beta$.
} 

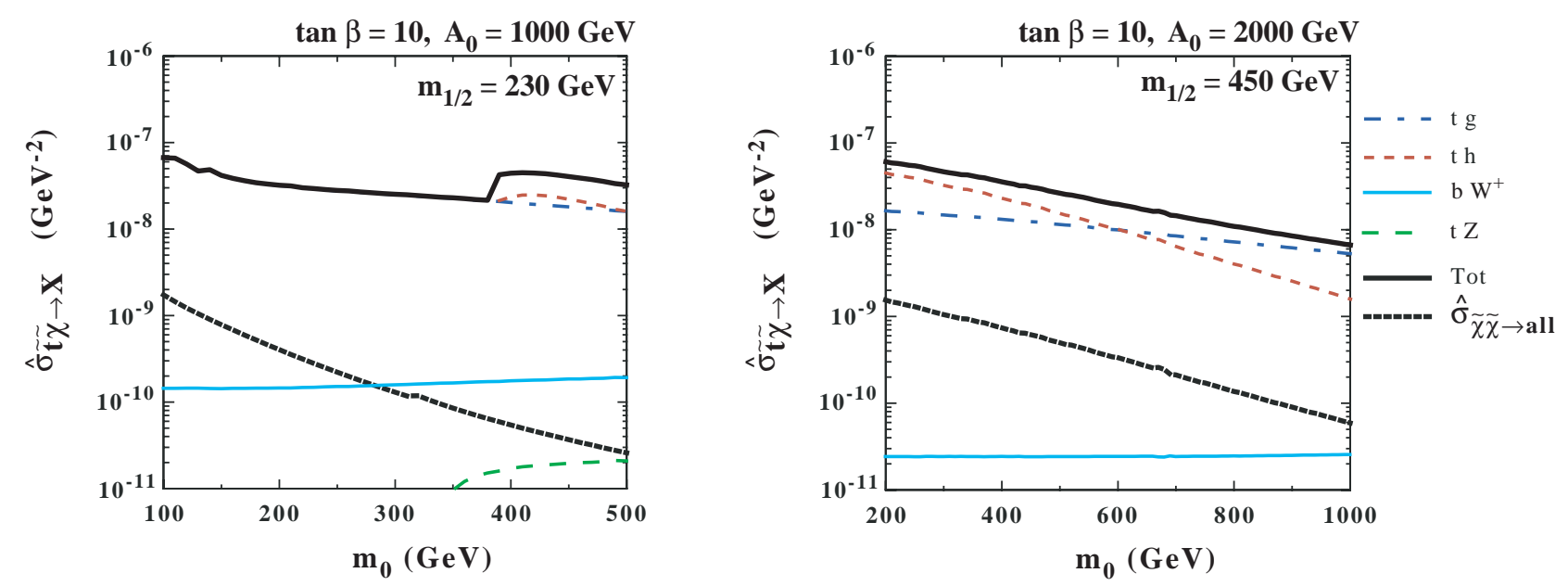

Figure 1: The separate contributions to the $\tilde{\chi} \tilde{t}_{1}$ coannihilation cross sections $\hat{\sigma} \equiv a+\frac{1}{2} b x$ for $x=T / m_{\tilde{\chi}}=1 / 23$ as functions of $m_{0}$ for (a) $m_{1 / 2}=230 \mathrm{GeV}, A_{0}=1000 \mathrm{GeV}$ and (b) $m_{1 / 2}=450 \mathrm{GeV}, A_{0}=2000 \mathrm{GeV}$. Also shown are the total cross section and, for comparison, the much smaller total cross section for $\tilde{\chi} \tilde{\chi}$ annihilation.

the neutralino. Thus, it is when $A_{0}$ is large that we expect $\tilde{\chi} \tilde{t}_{1}$ coannihilations to become important.

It is important to distinguish between the effective low-energy parameters $A_{t}$, etc., and the high-energy input parameter $A_{0}$, which are related through the running of the renormalization-group equations. For example, for $\tan \beta=10$ and $m_{0}=300 \mathrm{GeV}, \tilde{t}_{1} \tilde{\chi}$ coannihilations are important when $m_{1 / 2}=200,450$, and $670 \mathrm{GeV}$ and $A_{0}=1000,2000$, and $3000 \mathrm{GeV}$, but these values correspond to $A_{t} \simeq 565,1200$, and $1700 \mathrm{GeV}$ respectively. Furthermore, the values of $A$ for the light squarks are different and typically larger than $A_{t}$.

We display in Fig. 1 numerical values of the contributions to $\hat{\sigma} \equiv a+b x / 2$ (see (11)) in $\tilde{\chi} \tilde{t}_{1}$ coannihilation, for the representative values $x=1 / 23, \tan \beta=10, \mu>0$ and (a) $m_{1 / 2}=230 \mathrm{GeV}, A_{0}=1000 \mathrm{GeV}$ and (b) $m_{1 / 2}=450 \mathrm{GeV}, A_{0}=2000 \mathrm{GeV}$ as functions of $m_{0}$. For comparison, the total cross section for $\tilde{\chi} \tilde{\chi}$ annihilation to all final states is also shown, as a thick dotted line. We see that the $\tilde{\chi} \tilde{t}_{1} \rightarrow t g$ and $t h$ coannihilation cross sections dominate by large factors over the total $\tilde{\chi} \tilde{\chi}$ annihilation cross section, suggesting that they may have a greater importance than that suggested by simply comparing Boltzmann suppression factors. The feature in Fig. 1(a) at $m_{0} \sim 400 \mathrm{GeV}$ is due to the threshold for the production of $t h$ final states. At smaller values of $m_{0}$, this final state is kinematically forbidden.

Fig. 2 displays similar plots for $\tilde{t}_{1} \tilde{t}_{1}^{*}$ annihilation, for the same parameter choices as in Fig. 1. In this case, the dominant $\tilde{t}_{1} \tilde{t}_{1}^{*}$ annihilation cross sections are into $g g$ and $h h$, and even 

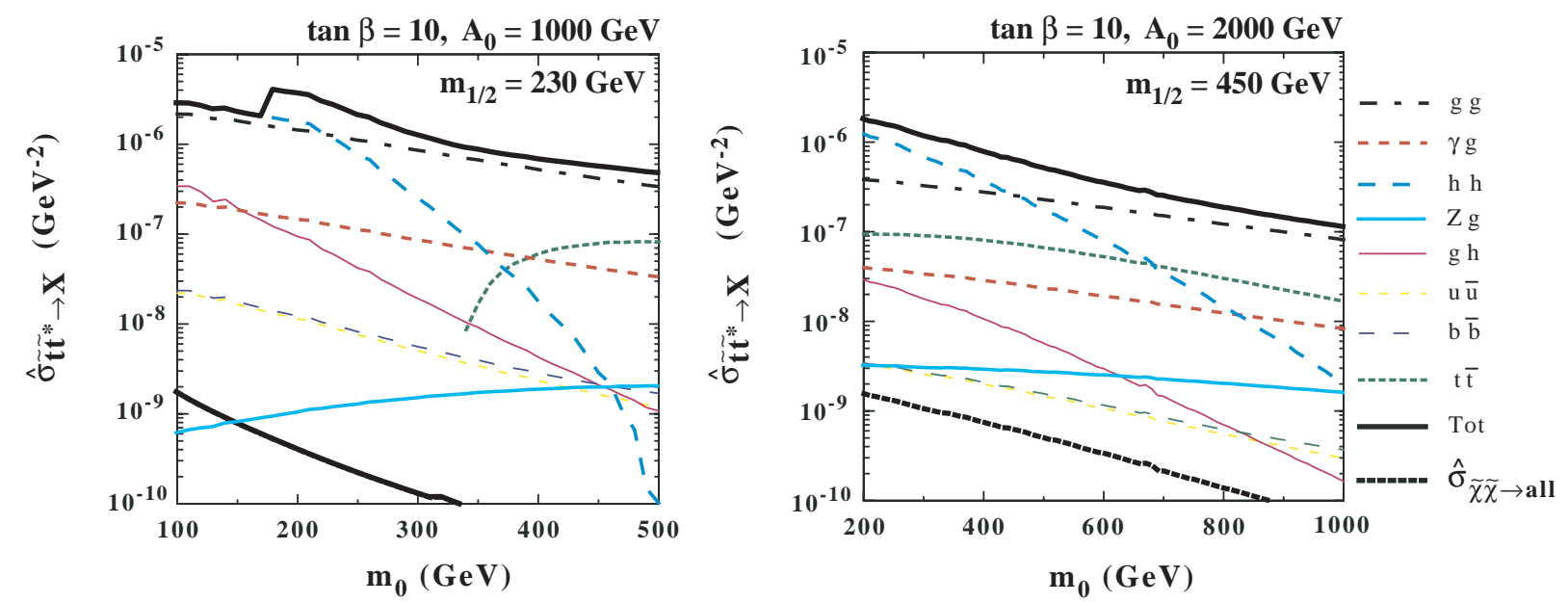

Figure 2: The separate contributions to the $\tilde{t}_{1} \tilde{t}_{1}^{*}$ annihilation cross sections $\hat{\sigma} \equiv a+\frac{1}{2} b x$ for $x=T / m_{\tilde{\chi}}=1 / 23$, as functions of $m_{0}$ for (a) $m_{1 / 2}=230 \mathrm{GeV}, A_{0}=1000 \mathrm{GeV}$ and $(b)$ $m_{1 / 2}=450 \mathrm{GeV}, A_{0}=2000 \mathrm{GeV}$. Also shown are the total cross section and, for comparison, the much smaller total cross section for $\tilde{\chi} \tilde{\chi}$ annihilation.

subdominant cross sections such as $\gamma g, Z g, g h$ and the various quark-antiquark channels are far larger than the total $\tilde{\chi} \tilde{\chi}$ annihilation cross section. Once again, when $A_{0}=1000 \mathrm{GeV}$ we see thresholds, in this case corresponding to $h h$ production at $m_{0} \sim 180 \mathrm{GeV}$ and $t \bar{t}$ production at $m_{0} \sim 330 \mathrm{GeV}$.

The $\tilde{t}_{1} \tilde{t}_{1}$ annihilation cross sections shown in Fig. 3 show that the cross section for annihilation into the $t t$ final state, when it is kinematically open, is also far larger than the total $\tilde{\chi} \tilde{\chi}$ annihilation cross section.

A complete study of coannihilation effects must include not only the $\tilde{\chi} \tilde{\ell}$ processes considered previously [7, [8], and the $\tilde{\chi} \tilde{t}_{1}$ processes considered above, but also $\tilde{\ell}_{1} \tilde{t}_{1}$ coannihilations. Accordingly, the final set of coannihilation cross sections we present are those for $\tilde{\ell}_{1} \tilde{t}_{1}$ and $\tilde{\ell}_{1}^{*} \tilde{t}_{1}$, shown in Fig. $⿴$. We see that, when (a) $A_{0}=1000 \mathrm{GeV}$, the $t \tau, t \bar{\tau}$ and $b \nu_{e}$ final states are the most important, followed by $t e, b \nu_{\tau}$ and $t \bar{e}$, whereas (b) the $t \bar{\tau}$ and $t \bar{e}$ final states are relatively much less important when $A_{0}=2000 \mathrm{GeV}$. In all panels of Fig. 目, there are coannihilation cross sections much greater than the total $\tilde{\chi} \tilde{\chi}$ annihilation cross section, which is also plotted.

The basic reason for the relatively small magnitude of the $\tilde{\chi} \tilde{\chi}$ annihilation cross section is that it is dominated by the $P$-wave suppressed cross sections for $\tilde{\chi} \tilde{\chi}$ annihilation to fermion pairs. This was also the basic reason why $\tilde{\chi} \tilde{\ell}$ coannihilation processes were previously found to be so important [7, 8, 9, [10]. 

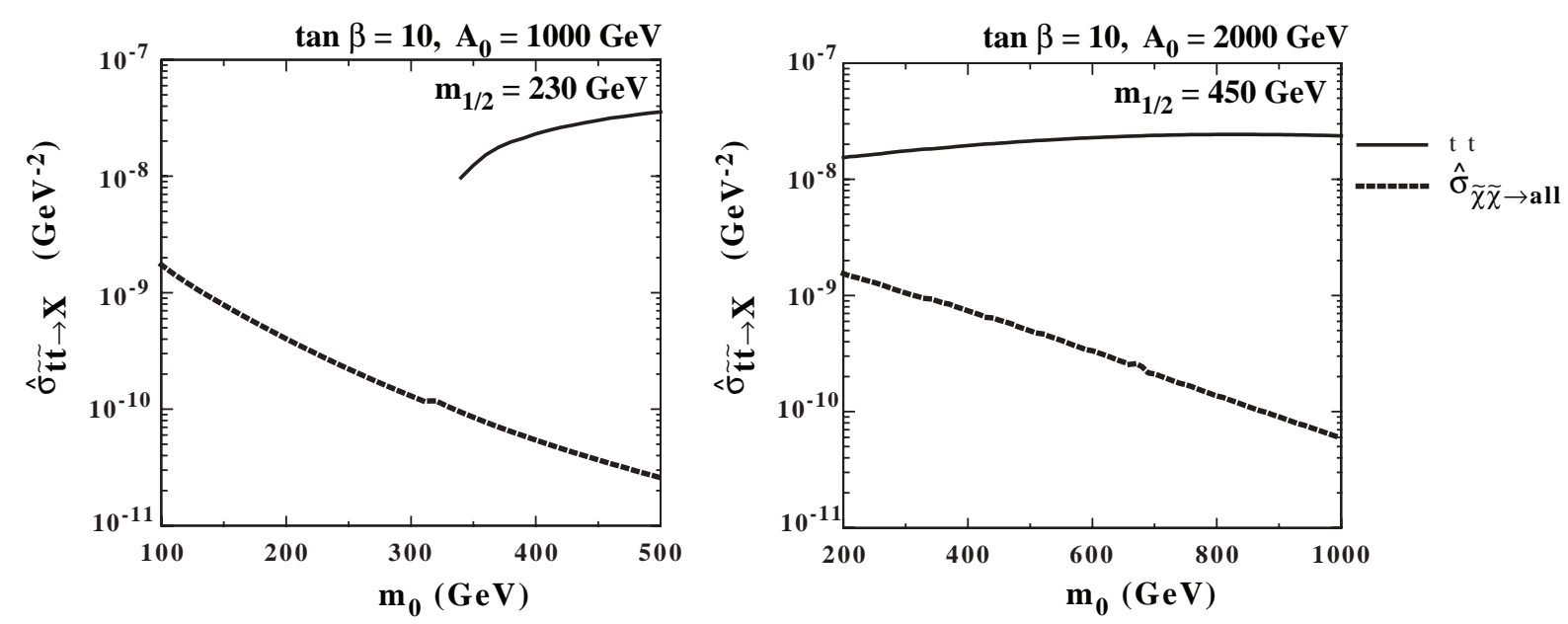

Figure 3: The $\tilde{t}_{1} \tilde{t}_{1} \rightarrow t$ annihilation cross sections $\hat{\sigma} \equiv a+\frac{1}{2} b x$ for $x=T / m_{\tilde{\chi}}=1 / 23$, as functions of $m_{0}$ for (a) $m_{1 / 2}=230 \mathrm{GeV}, A_{0}=1000 \mathrm{GeV}$ and (b) $m_{1 / 2}=450 \mathrm{GeV}$, $A_{0}=2000 \mathrm{GeV}$. Also shown, for comparison, is the much smaller total cross section for $\tilde{\chi} \tilde{\chi}$ annihilation.
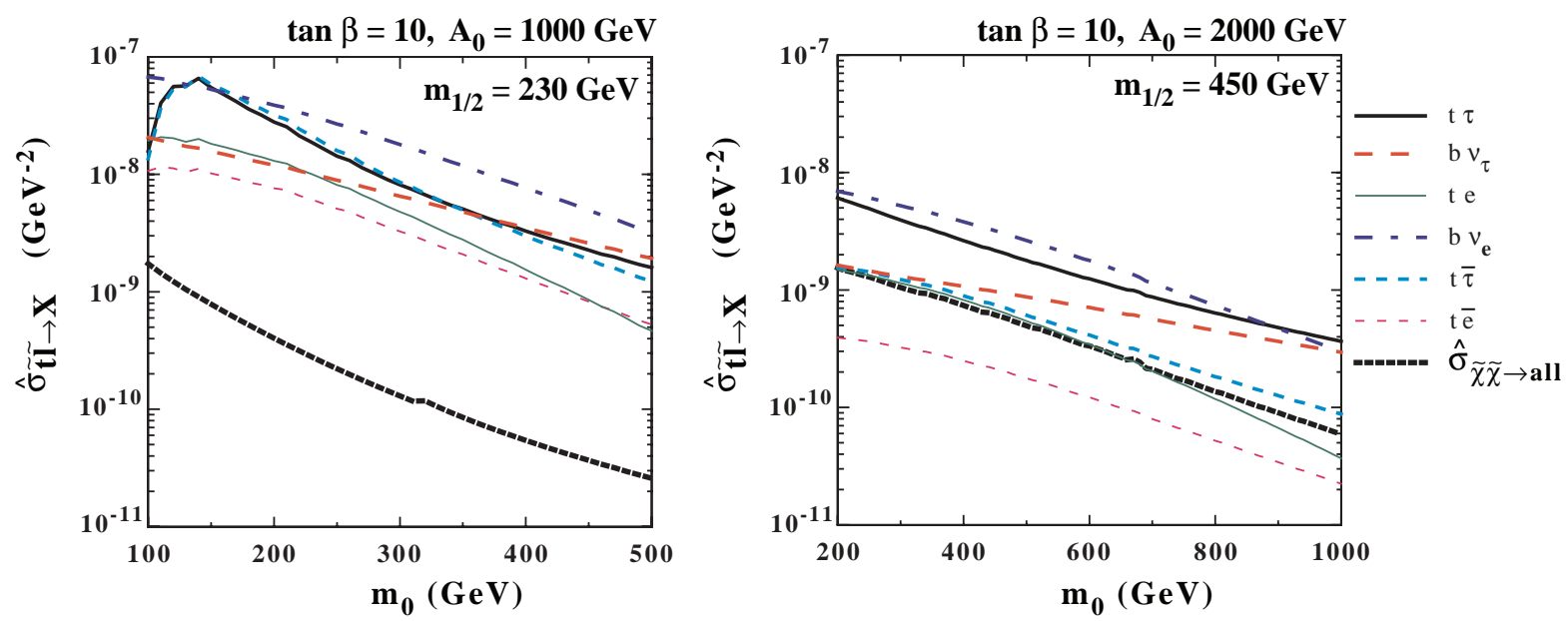

Figure 4: The separate contributions to the $\tilde{\ell}_{1} \tilde{t}_{1}$ coannihilation cross sections $\hat{\sigma} \equiv a+\frac{1}{2} b x$ for $x=T / m_{\tilde{\chi}}=1 / 23$, as functions of $m_{0}$ for (a) $m_{1 / 2}=230 \mathrm{GeV}, A_{0}=1000 \mathrm{GeV}$ and (b) $m_{1 / 2}=450 \mathrm{GeV}, A_{0}=2000 \mathrm{GeV}$. Also shown, for comparison, is the much smaller total cross section for $\tilde{\chi} \tilde{\chi}$ annihilation. 
The contributions of the various annihilation channels to $\sigma_{\text {eff }}$ are weighted by the relative abundances of the $\tilde{\chi}, \tilde{t}_{1}$ and $\tilde{\ell}_{1}$. For a stop degenerate with the $\tilde{\chi}, \tilde{t}_{1} \tilde{t}_{1}^{*}$ annihilation, $\tilde{t}_{1} \tilde{t}_{1}$ annihilation and $\tilde{\chi} \tilde{t}_{1}$ coannihilation are clearly the dominant contributions to $\Delta \sigma_{\text {eff }}$, and hence to $\sigma_{\text {eff }}$ in (6), and the final neutralino relic density is greatly reduced. As the stops become heavier than the neutralinos, their number densities are exponentially suppressed and the stop contributions to $\sigma_{\text {eff }}$ become less important. Fig. 5 shows the sizes of the separate contributions to $\hat{\sigma}_{\text {eff }}$ from $\tilde{\chi} \tilde{\chi}$ annihilation, $\tilde{t}_{1} \tilde{\chi}$ coannihilation and $\tilde{t}_{1} \tilde{t}_{1}^{*}, \tilde{t}_{1} \tilde{t}_{1}$ annihilations (combined), as functions of the mass difference between the $\tilde{t}_{1}$ and $\tilde{\chi}$. In Fig. 5, we have fixed $m_{0}=300 \mathrm{GeV}, \tan \beta=10, \mu>0, A_{0}=$ (a) 1000 and (b) $2000 \mathrm{GeV}$, and computed $\hat{\sigma}_{\text {eff }}$ for varying $m_{1 / 2}$, which amounts to varying the fractional mass difference $\Delta M \equiv\left(m_{\tilde{t}_{1}}-m_{\tilde{\chi}}\right) / m_{\tilde{\chi}}$. For these choices, the stau mass, $m_{\tilde{\tau}_{1}}$, is much larger than $m_{\tilde{\chi}}$. The thin dotted lined is the value of $\hat{\sigma}$ that one would compute if one ignored all coannihilation

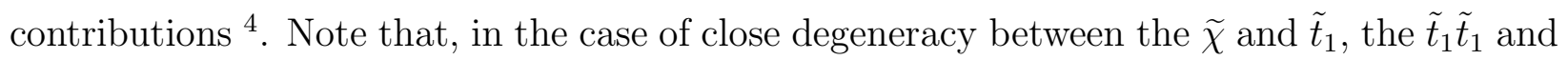
$\tilde{t}_{1} \tilde{t}_{1}^{*}$ annihilations dominate $\hat{\sigma}_{\text {eff }}$. However, since these contributions are suppressed by two powers of $n_{\text {eq, } \tilde{\mathrm{t}}_{1}}$, they drop rapidly with $\Delta M$, and neutralino-stop coannihilation takes over. For $A_{0}=2000 \mathrm{GeV}$, this occurs at $\Delta M \gtrsim 0.18$. This contribution in turn falls with one power of $n_{\mathrm{eq}, \tilde{\mathrm{t}}_{1}}$, and $\tilde{\chi} \tilde{\chi}$ annihilation re-emerges as the dominant reaction for $\Delta M \gtrsim 0.25$. When $\Delta M \gtrsim 0.35, \tilde{t}_{1}$ coannihilation effects can be neglected. In We see the presence of kinematic thresholds also in Fig. 5. In panel (a), we see the threshold for producing a single top quark in $\tilde{t} \tilde{\chi}$ coannihilations, and in panel (b) we see the threshold for producing a $t \bar{t}$ pair in $\tilde{\chi} \tilde{\chi}$ annihilations.

\section{Implications of $\tilde{t}_{1}$ Coannihilations for the Region of CMSSM Parameter Space Favoured by Cosmology}

We now explore the consequences of $\tilde{t}_{1}$ coannihilations for the region of CMSSM parameter space in which $0.1<\Omega_{\tilde{\chi}} h^{2}<0.3$, as favoured by cosmology. We display in Fig. 6 the $\left(m_{1 / 2}, m_{0}\right)$ planes for $\tan \beta=10$ and $\mu>0$, for the different values of $A_{0}=$ (a) 0 , (b) 1000 , (c) 2000 and (d) $3000 \mathrm{GeV}$. The very dark (red) shaded regions have $m_{\tilde{\tau}_{1}}$ or $m_{\tilde{t}_{1}}<m_{\tilde{\chi}}$, and hence are excluded by the very stringent bounds on charged dark matter [1]. The light (turquoise) shaded regions correspond to the preferred relic-density range $0.1<\Omega_{\tilde{\chi}} h^{2}<0.3$. The dark (green) shaded regions are those excluded by measurements of $b \rightarrow s \gamma$. The intermediate (pink) shaded regions in panels (a) and (b) are those favoured by the BNL

\footnotetext{
${ }^{4}$ This differs from $\hat{\sigma}_{\tilde{\chi} \chi}$ because of the number-density weighting factor.
} 

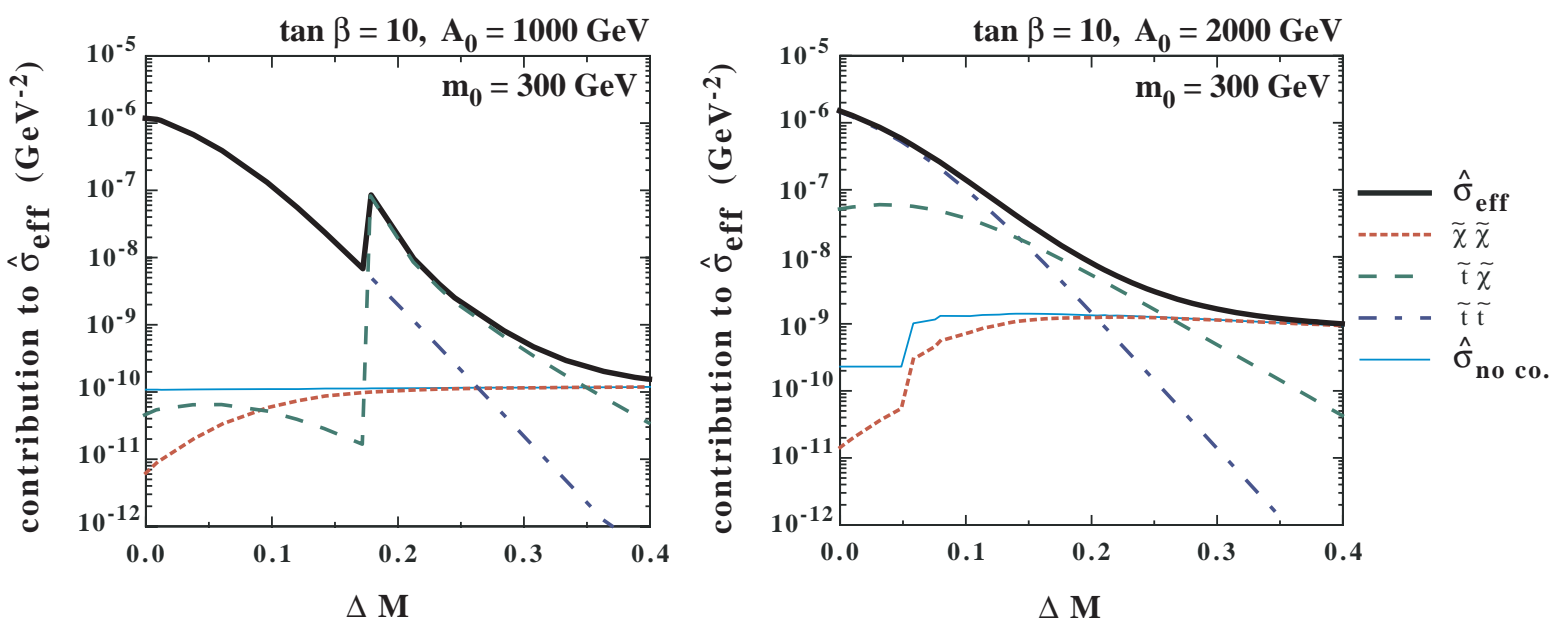

Figure 5: The separate contributions to the total effective cross sections $\hat{\sigma}_{\text {eff }}$ for $x=T / m_{\tilde{\chi}}=$ $1 / 23$, as functions of $\Delta M \equiv\left(m_{\tilde{\tau}_{R}}-m_{\tilde{\chi}}\right) / m_{\tilde{\chi}}$, obtained by varying $m_{1 / 2}$, with (a) $A_{0}=$ $1000 \mathrm{GeV}$ and (b) $A_{0}=2000 \mathrm{GeV}$, both for $\tan \beta=10, \mu>0$ and $m_{0}=300 \mathrm{GeV}$.

measurement of $g_{\mu}-2$ at the 2- $\sigma$ level [17]. The (black) dashed line in panel (a) is the contour $m_{\widetilde{\chi}^{ \pm}}=104 \mathrm{GeV}$, corresponding to the kinematic range of LEP 2, and the (red) dotted line is the contour $m_{h}=114 \mathrm{GeV}$, as evaluated using FeynHiggs [18, corresponding to the LEP lower limit on the mass of the Higgs boson. These contours are also glimpsed in the other panels.

Panel (a) of Fig. 6 is indistinguishable from analogous plots shown previously [8, 12]: $\tilde{t}_{1}$ coannihilations are not important when $A_{0}=0$. Panel (b) shows how $\tilde{t}_{1}$ coannihilation generates a 'tail' of allowed CMSSM parameter space, extending the 'bulk' region of the $\left(m_{1 / 2}, m_{0}\right)$ plane up as far as $m_{0} \sim 450 \mathrm{GeV}$. The boundary of the region where $m_{\tilde{t}_{1}}<m_{\tilde{\chi}}$, and hence the $\tilde{t}_{1} \tilde{\chi}$ coannihilation region, slopes to the left as $m_{0}$ is increased, since we have fixed $A_{0}$ over the plane. As $m_{0}$ is increased, the impact of the $A$-dependent off-diagonal term is diminished and the light stop is heavier. To compensate for this, one must decrease $m_{1 / 2}$ to obtain the necessary degree of degeneracy between $\tilde{t}_{1}$ and $\tilde{\chi}$. This effect is also seen in panels (c) and (d) of Fig. 国.

In the particular cae of $A_{0}=1000 \mathrm{GeV}$ shown in panel (b) of Fig. 6, the $\tilde{t}_{1}$ coannihilation tail happens to be excluded by $b \rightarrow s \gamma$. However, this is not the case for (c) $A_{0}=2000 \mathrm{GeV}$ and (d) $A_{0}=3000 \mathrm{GeV}$, where the $\tilde{t}_{1}$ coannihilation tail extends up to $m_{0} \sim 900$ and beyond $1350 \mathrm{GeV}$, respectively. For (b) $A_{0}=2000 \mathrm{GeV}$, the region favoured by $g_{\mu}-2$ is hidden on the left, inside the excluded region. However, around $A_{0}=1500 \mathrm{GeV}$ there is a $\tilde{\chi} \tilde{t}_{1}$ coannihilation region that satisfies both the $b \rightarrow s \gamma$ and $g_{\mu}-2$ constraints.

The cosmologically-favoured region is broadened significantly when $t \bar{t}$ production is kine- 

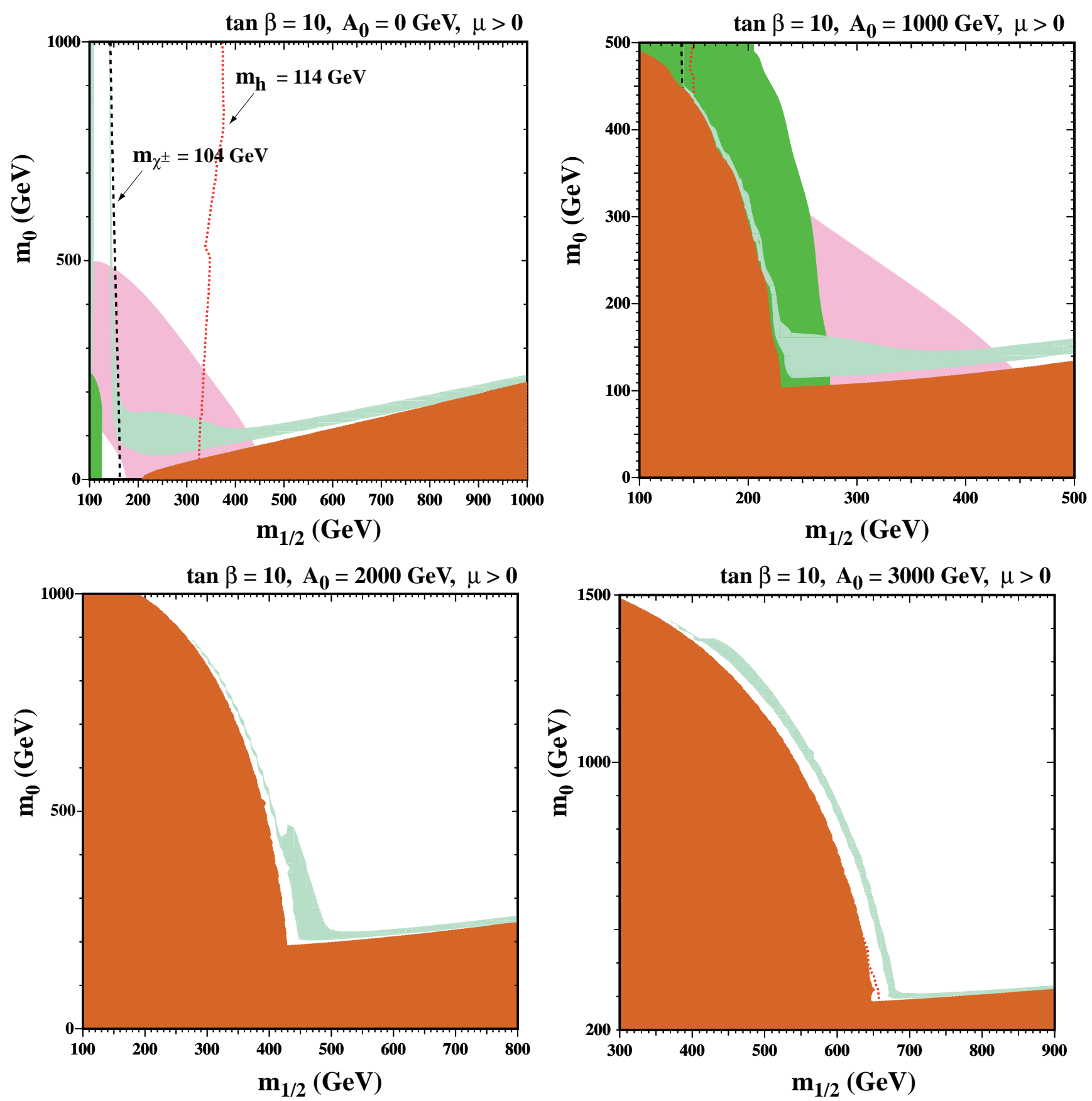

Figure 6: The $\left(m_{1 / 2}, m_{0}\right)$ planes for $\tan \beta=10, \mu>0$ and $A_{0}=$ (a) 0, (b) 1000, (c) 2000 and (d) $3000 \mathrm{GeV}$. The very dark (red) shaded regions are excluded because the LSP is the $\tilde{t}_{1}$ or the $\tilde{\tau}_{1}$. The dark shaded regions are excluded by $b \rightarrow s \gamma$. The intermediate shaded regions are favoured by $g_{\mu}-2$ at the 2- $\sigma$ level. The light (turquoise) shaded regions are those favoured by cosmology, with $0.1 \leq \Omega_{\tilde{\chi}} h^{2} \leq 0.3$ after the inclusion of coannihilation effects. The (black) dashed lines are the contours $m_{\tilde{\chi}^{ \pm}}=104 \mathrm{GeV}$, and the (red) dotted lines are the contours $m_{h}=114 \mathrm{GeV}$. 
matically allowed in $\tilde{\chi} \widetilde{\chi}$ annihilation The t-channel stop exchange contribution to this process is significantly enhanced when $\tilde{t}_{1}$ is relatively light: $m_{\tilde{t}_{1}} \simeq m_{\tilde{\chi}}$. This feature can be seen in Fig. 6(c) and Fig. 6(d). The $\tilde{t}_{1} \tilde{\ell}$ coannihilations are only important in the corner area near the instep of the dark (red) shaded region where $m_{\tilde{\tau}_{1}} \simeq m_{\tilde{t}_{1}} \simeq m_{\tilde{\chi}}$.

Fig. 7 shows analogous $\left(m_{1 / 2}, m_{0}\right)$ planes for the larger values $\tan \beta=20$ and 30 , both again for $\mu>0$. We see in panel (a) that the $\tilde{t}_{1}$ coannihilation tail extends up to $m_{0} \sim$ $900 \mathrm{GeV}$ when $\tan \beta=20$ and $A_{0}=2000 \mathrm{GeV}$, beyond the region forbidden by $b \rightarrow s \gamma$. We then see in panel (b) that the $\tilde{t}_{1}$ coannihilation tail extends beyond $m_{0} \sim 1400 \mathrm{GeV}$ when $\tan \beta=20$ and $A_{0}=3000 \mathrm{GeV}$. A similar portion of the $\tilde{t}_{1}$ coannihilation tail that is consistent with $b \rightarrow s \gamma$ is also visible in panel (c), for $\tan \beta=20$ and $A_{0}=2000 \mathrm{GeV}$, where $m_{0} \sim 900 \mathrm{GeV}$. For fixed $A_{0}$, as $\tan \beta$ is increased the $b \rightarrow s \gamma$ constraint becomes more severe.

The $\tilde{t}_{1}$ coannihilation tails do not increase the allowed range of $m_{1 / 2}$, as was the case for the $\tilde{\ell}$ coannihilation tail [7, 8, 9, 10], but they do add a significant filament to the CMSSM region allowed by experiment and favoured by cosmology.

In the above illustrative examples, we have chosen the sign of $A_{0}$ to be the same as that of $\mu$, with the effect of maximizing the stop off-diagonal mass terms, and hence minimizing $m_{\tilde{t}_{1}}$. If the sign of $A_{0}$ is switched while keeping $\mu>0$, an analogous $\tilde{\chi} \tilde{t}_{1}$ coannihilation region is found only at even larger negative $A_{0}$. However, the $m_{h}$ constraint is more severe for $A_{0}<0$, and also for $\mu<0$, excluding the region of parameter space of interest in the $\tilde{\chi} \tilde{t}_{1}$ coannihilation context.

We have not discussed in this paper the potential constraint imposed by the absence of colour and charge breaking (CCB) vacua, or at least the suppression of transitions to $\mathrm{CCB}$ vacua. This constraint would restrict the value of $A_{0}$ relative to $m_{0}$ [19]. If the CCB constraint is imposed, some of the parameter space for small $m_{0}$ will be excluded, but there will still be regions at high $m_{0}$ where $\tilde{\chi} \tilde{t}_{1}$ coannihilation is crucial. The issue of compatibity with the potential $g_{\mu}-2$ constraint would then arise. Since raising $m_{0}$ suppresses the neutralino-proton elastic scatttering cross section, $\tilde{\chi} \tilde{t}_{1}$ coannihilation would tend to lower the neutralino direct detection rate for the same range of $m_{\tilde{\chi}}$.

\section{Conclusions and Open Issues}

We have documented in this paper the potential importance of $\tilde{\chi} \tilde{t}_{1}$ coannihilation in delineating the preferred domain of CMSSM parameter space for $A_{0} \neq 0$. In this paper, we have only sctratched the surface of this subject, whose higher-dimensional parameter space merits 

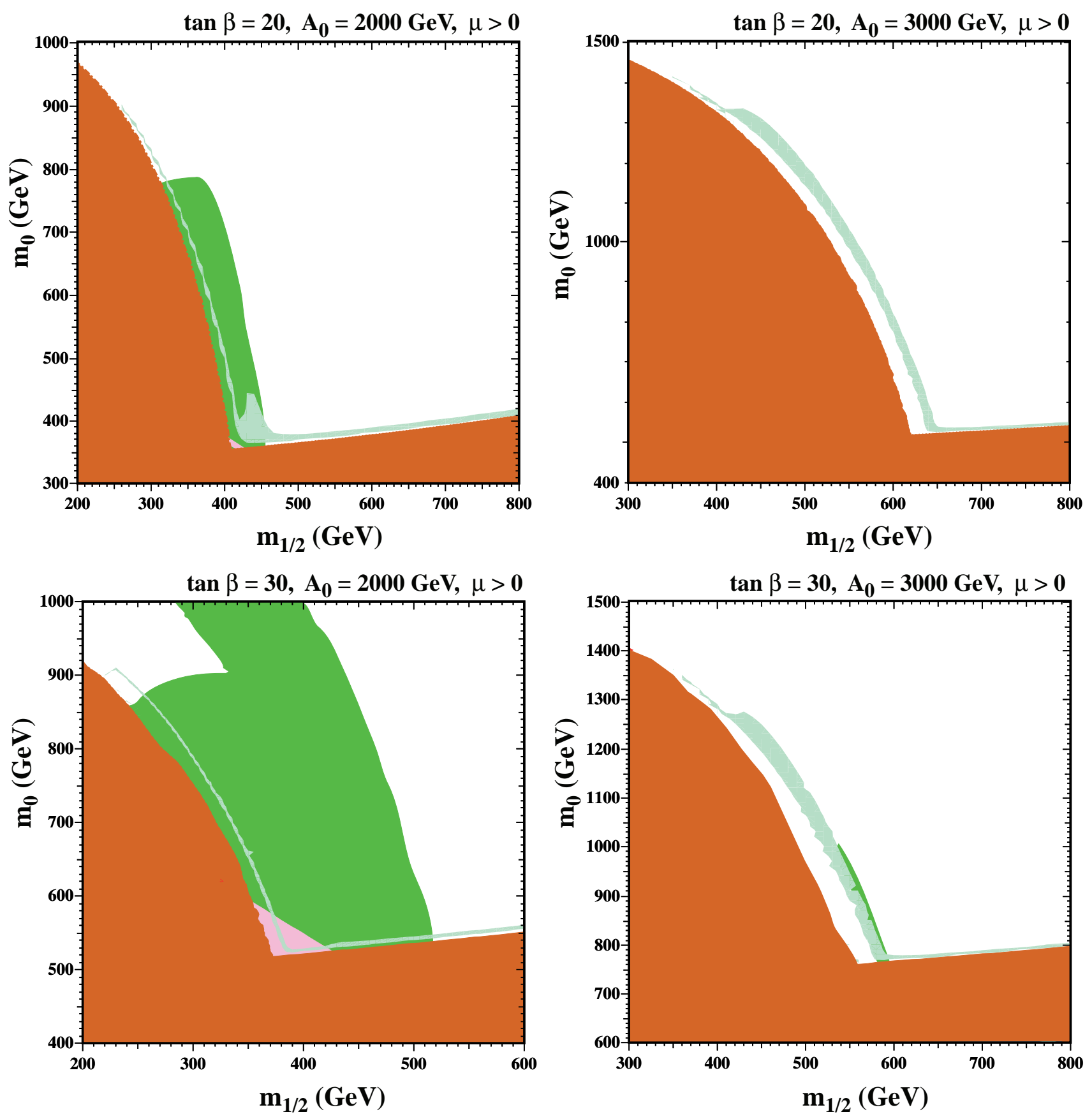

Figure 7: Notation as for Fig. 6. Panels $(a, b)$ are for $\tan \beta=20, \mu>0$, and panels $(c, d)$ are for $\tan \beta=30, \mu>0$. Panels (a,c) are for $A_{0}=2000 \mathrm{GeV}$, and panels (b,d) for $A_{0}=3000 \mathrm{GeV}$. 
more detailed exploration. The Appendix provides details of the diagrammatic calculations that should be sufficient for our results to be verified and used by other authors. Although applied in the context of the CMSSM, our results may also be used in more general MSSM contexts. However, other coannihilation processes are also important in other regions of the general MSSM parameter space. For example, in the CMSSM the sbottom mass is generally larger than the stop mass even for large $\tan \beta$. However, if one allow non-degeneracy in the scalar soft breaking mass term, a sbottom NLSP becomes possible [10]. A complete calculation of the LSP relic density in the MSSM requires a careful discussion of all such coannihilation possibilities.

\section{Acknowledgments}

We thank Toby Falk for many related discussions. The work of K.A.O. and Y.S. was supported in part by DOE grant DE-FG02-94ER-40823. 


\section{Appendix}

We calculate the tree contributions to the coannihilation and annihilation amplitudes that are leading order in $\alpha_{s}, \alpha_{t} \equiv \lambda_{t}^{2} / 4 \pi$ and/or $\alpha_{b} \equiv \lambda_{b}^{2} / 4 \pi$. We keep terms that are of the same order in $\sqrt{\alpha_{s} \alpha_{e m}}, \sqrt{\alpha_{t} \alpha_{e m}}, \sqrt{\alpha_{b} \alpha_{e m}}, \sqrt{\alpha_{s} \alpha_{W}}, \sqrt{\alpha_{t} \alpha_{W}}$ and/or $\sqrt{\alpha_{b} \alpha_{W}}$, but neglect most terms in $\alpha_{e m}$ and/or $\alpha_{W}$. This approximation is motivated by the relatively large magnitudes of $\alpha_{s}$ and $\alpha_{t}$, and the fact that $\alpha_{b} \sim \alpha_{t}$ at large $\tan \beta$. We have however included s-channel $Z$, and $\gamma$ exchange for $\tilde{t}_{1} \tilde{t}_{1}^{*} \rightarrow t \bar{t}$ and checked that they make negligible contribution to the total cross section and therefore neglect these channels in the remaining amplitudes.

\section{Couplings}

Here we list couplings used in the calculation. Factors not written, such as $i, \gamma$ 's, QCD generators and momentum, are taken into account in the calculation of amplitudes squared, which are listed subsequently.

$$
\begin{aligned}
& C_{g-g-g}=-g_{s} \\
& C_{\tilde{t}_{1}-\tilde{t}_{1}-g}=-g_{s} \\
& C_{\tilde{t}_{1}-\tilde{t}_{1}-g-g}=g_{s}^{2} \\
& C_{\tilde{t}_{1}-\tilde{t}_{1}-\gamma}=-2 e / 3 \\
& C_{\tilde{t}_{1}-\tilde{t}_{1}-\gamma-g}=4 \text { e } g_{s} / 3 \\
& C_{\tilde{t}_{1}-\tilde{t}_{1}-Z}=\frac{-g_{2}}{\cos \theta_{W}}\left((-2 / 3) \sin ^{2} \theta_{W}+\cos ^{2} \theta_{t} / 2\right) \\
& C_{\tilde{t}_{1}-\tilde{t}_{2}-Z}=\frac{g_{2}}{\cos \theta_{W}}(1 / 2) \cos \theta_{t} \sin \theta_{t} \\
& C_{\tilde{t}_{1}-\tilde{t}_{1}-Z-g}=\frac{2 g_{s} g_{2}}{\cos \theta_{W}}\left((1 / 2) \cos ^{2} \theta_{t}-(2 / 3) \sin ^{2} \theta_{W}\right) \\
& C_{q-q-g}=-g_{s} \\
& C_{\tilde{t}_{1}-t-\tilde{g}}^{L}=\sqrt{2} g_{s} \sin \theta_{t} \\
& C_{\tilde{t}_{1}-t-\tilde{g}}^{R}=-\sqrt{2} g_{s} \cos \theta_{t} \\
& C_{\tilde{t}_{1}-\tilde{t}_{1}-h}=\frac{g_{2} m_{Z}}{\cos \theta_{W}}\left(\left(\frac{1}{2}-\frac{2}{3} \sin ^{2} \theta_{W}\right) \cos ^{2} \theta_{t}+\frac{2}{3} \sin ^{2} \theta_{W} \sin ^{2} \theta_{t}\right) \sin (\alpha+\beta) \\
& -\frac{g_{2} m_{t}^{2} \cos \alpha}{m_{W} \sin \beta}-\frac{g_{2} m_{t}\left(\mu \sin \alpha-A_{t} \cos \alpha\right)}{m_{W} \sin \beta} \sin \theta_{t} \cos \theta_{t} \\
& C_{\tilde{t}_{1}-\tilde{t}_{2}-h}=\frac{g_{2} m_{Z}}{\cos \theta_{W}}\left(\frac{2}{3} \sin ^{2} \theta_{W}-\left(\frac{1}{2}-\frac{2}{3} \sin ^{2} \theta_{W}\right)\right) \cos \theta_{t} \sin \theta_{t} \sin (\alpha+\beta) \\
& -\frac{g_{2} m_{t}\left(\mu \sin \alpha-A_{t} \cos \alpha\right)}{2 m_{W} \sin \beta}\left(\cos ^{2} \theta_{t}-\sin ^{2} \theta_{t}\right)
\end{aligned}
$$




$$
\begin{aligned}
C_{\tilde{t}_{1}-\tilde{t}_{1}-H}= & -\frac{g_{2} m_{Z}}{\cos \theta_{W}}\left(\left(\frac{1}{2}-\frac{2}{3} \sin ^{2} \theta_{W}\right) \cos ^{2} \theta_{t}+\frac{2}{3} \sin ^{2} \theta_{W} \sin ^{2} \theta_{t}\right) \cos (\alpha+\beta) \\
& -\frac{g_{2} m_{t}^{2} \sin \alpha}{m_{W} \sin \beta}+\frac{g_{2} m_{t}\left(\mu \cos \alpha+A_{t} \sin \alpha\right)}{m_{W} \sin \beta} \sin \theta_{t} \cos \theta_{t} \\
C_{\tilde{t}_{1}-\tilde{t}_{2}-H}= & -\frac{g_{2} m_{Z}}{\cos \theta_{W}}\left(\frac{2}{3} \sin ^{2} \theta_{W}-\left(\frac{1}{2}-\frac{2}{3} \sin ^{2} \theta_{W}\right)\right) \sin \theta_{t} \cos \theta_{t} \cos (\alpha+\beta) \\
& +\frac{g_{2} m_{t}\left(\mu \cos \alpha+A_{t} \sin \alpha\right)}{2 m_{W} \sin \beta}\left(\cos ^{2} \theta_{t}-\sin ^{2} \theta_{t}\right) \\
C_{\tilde{t}_{1}-\tilde{t}_{1}-A}= & 0 \\
C_{\tilde{t}_{1}-\tilde{t}_{2}-A}= & \frac{g_{2} m_{t}}{2 m_{W}}\left(\mu-A_{t} \cot \beta\right) \\
C_{t-t-\gamma}= & -2 e / 3 \\
C_{f-f-Z}^{L}= & \frac{g_{2}}{\cos \theta_{W}}\left(Q_{f} \sin \theta_{W}-T_{3 f}\right) \\
C_{f-f-Z}^{R}= & \frac{g_{2}}{\cos \theta_{W}} Q_{f} \sin \theta_{W} \\
C_{t-t-h}= & -\frac{g_{2} m_{t} \cos \alpha}{2 m_{W} \sin \beta} \\
C_{t-t-H}= & -\frac{g_{2} m_{t} \sin \alpha}{2 m_{W} \sin \beta} \\
C_{t-t-A}= & \frac{g_{2} m_{t} \cot \beta}{2 m_{W}} \\
C_{b-b-h}= & \frac{g_{2} m_{b} \sin \alpha}{2 m_{W} \cos \beta} \\
C_{b-b-H}= & -\frac{g_{2} m_{b} \cos \alpha}{2 m_{W} \cos \beta} \\
C_{\tilde{t}_{1}-b-\chi_{i}^{+}}^{L}= & \frac{g_{2} m_{b}}{\sqrt{2} m_{W} \cos \beta} U_{i 2} \cos \theta_{t} \\
C_{\tilde{t}_{1}-b-\chi_{i}^{+}}^{R}= & -g_{2} V_{i 1} \cos \theta_{t}+\frac{g_{2} m_{t}}{\sqrt{2} m_{W} \sin \beta} V_{i 2} \sin \theta_{t} \\
C_{\tilde{\ell}_{1}-\nu-\chi_{i}^{+}}^{L}= & 0 \\
C_{\tilde{\ell}_{1}-\nu-\chi_{i}^{+}}^{R}= & \frac{g_{2} m_{\ell}}{\sqrt{2} m_{W} \cos \beta} U_{i 2} \sin \theta_{\ell}-g_{2} U_{i 1} \cos \theta_{\ell} \\
C_{h-h-h}= & -\frac{3 g_{2} m_{Z}}{2 \cos \theta_{W}} \cos 2 \alpha \sin (\beta+\alpha) \\
C_{h-h-H}= & -\frac{g_{2} m_{Z}}{2 \cos \theta_{W}}(2 \sin 2 \alpha \sin (\beta+\alpha)-\cos (\beta+\alpha) \cos 2 \alpha) \\
C_{h-H-H}= & \frac{g_{2} m_{Z}}{2 \cos \theta_{W}}(2 \sin 2 \alpha \cos (\beta+\alpha)+\sin (\beta+\alpha) \cos 2 \alpha) \\
C_{H-H}= & -\frac{3 g_{2} m_{Z}}{2 \cos \theta_{W}} \cos 2 \alpha \cos (\beta+\alpha) \\
& \\
&
\end{aligned}
$$




$$
\begin{aligned}
& C_{h-A-A}=-\frac{g_{2} m_{Z}}{2 \cos \theta_{W}} \cos 2 \beta \sin (\beta+\alpha) \\
& C_{H-A-A}=\frac{g_{2} m_{Z}}{2 \cos \theta_{W}} \cos 2 \beta \cos (\beta+\alpha) \\
& C_{h-H^{+-} H^{-}}=-g_{2}\left(m_{W} \sin (\beta-\alpha)+m_{Z} /\left(2 \cos \theta_{W}\right) \cos (2 \beta) \sin (\beta+\alpha)\right) \\
& C_{H-H^{+-} H^{-}}=-g_{2}\left(m_{W} \cos (\beta-\alpha)-m_{Z} /\left(2 \cos \theta_{W}\right) \cos (2 \beta) \cos (\beta+\alpha)\right) \\
& C_{h-Z-A}=g_{2} \cos (\alpha-\beta) /\left(2 \cos \theta_{W}\right) \\
& C_{H-Z-A}=g_{2} \sin (\alpha-\beta) /\left(2 \cos \theta_{W}\right) \\
& C_{h-W^{+}-H^{-}}=\frac{g_{2}}{2} \cos (\alpha-\beta) \\
& C_{H-W^{+}-H^{-}}=\frac{g_{2}}{2} \sin (\alpha-\beta) \\
& C_{\tilde{t}_{1}-\tilde{t}_{1}-h-h}=\frac{g_{2}^{2}}{2}\left(\frac{\cos 2 \alpha}{\cos ^{2} \theta_{W}}\left(\left(\frac{1}{2}-\frac{2}{3} \sin ^{2} \theta_{W}\right) \cos ^{2} \theta_{t}+\frac{2}{3} \sin ^{2} \theta_{W} \sin ^{2} \theta_{t}\right)-\frac{m_{t}^{2}}{m_{W}^{2}} \frac{\cos ^{2} \alpha}{\sin ^{2} \beta}\right) \\
& C_{\tilde{t}_{1}-\tilde{t}_{1}-h-H}=\frac{g_{2}^{2} \sin 2 \alpha}{2}\left(\left(\frac{1}{2}-\frac{2}{3} \sin ^{2} \theta_{W}\right) \sec ^{2} \theta_{W} \cos ^{2} \theta_{t}+\frac{2}{3} \tan ^{2} \theta_{W} \sin ^{2} \theta_{t}\right. \\
& \left.-\frac{m_{t}^{2}}{2 m_{W}^{2} \sin ^{2} \beta}\right) \\
& C_{\tilde{t}_{1}-\tilde{t}_{1}-H-H}=\frac{g_{2}^{2}}{2}\left(\frac{-\cos 2 \alpha}{\cos ^{2} \theta_{W}}\left(\left(\frac{1}{2}-\frac{2}{3} \sin ^{2} \theta_{W}\right) \cos ^{2} \theta_{t}+\frac{2}{3} \sin ^{2} \theta_{W} \sin ^{2} \theta_{t}\right)-\frac{m_{t}^{2}}{m_{W}^{2}} \frac{\sin ^{2} \alpha}{\sin ^{2} \beta}\right) \\
& C_{\tilde{t}_{1}-\tilde{t}_{1}-A-A}=\frac{g_{2}^{2}}{2}\left(\frac{\cos 2 \beta}{\cos ^{2} \theta_{W}}\left(\left(\frac{1}{2}-\frac{2}{3} \sin ^{2} \theta_{W}\right) \cos ^{2} \theta_{t}+\frac{2}{3} \sin ^{2} \theta_{W} \sin ^{2} \theta_{t}\right)-\frac{m_{t}^{2}}{m_{W}^{2} \tan ^{2} \beta}\right) \\
& C_{\tilde{t}_{1}-\tilde{t}_{1}-H^{+}-H^{-}}=\frac{g_{2}^{2} \cos 2 \beta}{2}\left(\left(-1+\left(\frac{1}{2}-\frac{2}{3} \sin ^{2} \theta_{W}\right) \sec ^{2} \theta_{W}\right) \cos ^{2} \theta_{t}+\frac{2}{3} \tan ^{2} \theta_{W} \sin ^{2} \theta_{t}\right) \\
& -\frac{g_{2}^{2}}{2 m_{W}^{2}}\left(m_{b}^{2} \tan ^{2} \beta \cos ^{2} \theta_{t}+m_{t}^{2} \cot ^{2} \beta \sin ^{2} \theta_{t}\right) \\
& C_{\tilde{t}_{1}-\tilde{b}_{1}-H^{+}}=-\frac{g_{2} m_{W}}{\sqrt{2}}\left(\sin 2 \beta-\frac{m_{b}^{2} \tan \beta+m_{t}^{2} \cot \beta}{m_{W}^{2}}\right) \cos \theta_{t} \cos \theta_{b}+\frac{g_{2}}{\sqrt{2} m_{W}} \\
& \times\left(m_{t} m_{b}(\tan \beta+\cot \beta) \sin \theta_{t} \sin \theta_{b}-m_{b}\left(A_{b} \tan \beta-\mu\right) \cos \theta_{t} \sin \theta_{b}\right. \\
& \left.-m_{t}\left(A_{t} \cot \beta-\mu\right) \sin \theta_{t} \cos \theta_{b}\right) \\
& C_{\tilde{t}_{1}-\tilde{b}_{2}-H^{+}}=\frac{g_{2} m_{W}}{\sqrt{2}}\left(\sin 2 \beta-\frac{m_{b}^{2} \tan \beta+m_{t}^{2} \cot \beta}{m_{W}^{2}}\right) \cos \theta_{t} \sin \theta_{b}+\frac{g_{2}}{\sqrt{2} m_{W}} \\
& \times\left(m_{t} m_{b}(\tan \beta+\cot \beta) \sin \theta_{t} \cos \theta_{b}-m_{b}\left(A_{b} \tan \beta-\mu\right) \cos \theta_{t} \cos \theta_{b}\right. \\
& \left.+m_{t}\left(A_{t} \cot \beta-\mu\right) \sin \theta_{t} \sin \theta_{b}\right) \\
& C_{\tilde{t}_{1}-\tilde{b}_{1}-W^{+}}=\frac{-g_{2}}{\sqrt{2}} \cos \theta_{t} \cos \theta_{b} \\
& C_{\tilde{t}_{1}-\tilde{b}_{2}-W^{+}}=\frac{g_{2}}{\sqrt{2}} \cos \theta_{t} \sin \theta_{b}
\end{aligned}
$$




$$
\begin{aligned}
& C_{\tilde{t}_{1}-t-\tilde{\chi}_{i}^{0}}^{L}=\frac{-g_{2}}{\sqrt{2}}\left(\cos \theta_{t}\left(N_{i 2}+\frac{\tan \theta_{W}}{3} N_{i 1}\right)+\sin \theta_{t} \frac{m_{t} N_{i 4}}{m_{W} \sin \beta}\right) \\
& C_{\tilde{t}_{1}-t-\tilde{\chi}_{i}^{0}}^{R}=\frac{-g_{2}}{\sqrt{2}}\left(\cos \theta_{t} \frac{m_{t} N_{i 4}}{m_{W} \sin \beta}-\frac{4}{3} \sin \theta_{t} \tan \theta_{W} N_{i 1}\right) \operatorname{sign}\left(m_{\tilde{\chi}_{i}^{0}}\right) \\
& C_{\tilde{t}_{2}-t-\tilde{\chi}_{i}^{0}}^{L}=\frac{-g_{2}}{\sqrt{2}}\left(-\sin \theta_{t}\left(N_{i 2}+\frac{\tan \theta_{W}}{3} N_{i 1}\right)+\cos \theta_{t} \frac{m_{t} N_{i 4}}{m_{W} \sin \beta}\right) \\
& C_{\tilde{t}_{2}-t-\tilde{\chi}_{i}^{0}}^{R}=\frac{-g_{2}}{\sqrt{2}}\left(-\sin \theta_{t} \frac{m_{t} N_{i 4}}{m_{W} \sin \beta}-\frac{4}{3} \cos \theta_{t} \tan \theta_{W} N_{i 1}\right) \operatorname{sign}\left(m_{\tilde{\chi}_{i}^{0}}\right) \\
& C_{\tilde{b}_{1}-b-\tilde{\chi}_{i}^{0}}^{L}=\frac{-g_{2}}{\sqrt{2}}\left(-\cos \theta_{b}\left(N_{i 2}-\frac{\tan \theta_{W}}{3} N_{i 1}\right)+\sin \theta_{b} \frac{m_{b} N_{i 3}}{m_{W} \cos \beta}\right) \\
& C_{\tilde{b}_{1}-b-\tilde{\chi}_{i}^{0}}^{R}=\frac{-g_{2}}{\sqrt{2}}\left(\cos \theta_{b} \frac{m_{b} N_{i 3}}{m_{W} \cos \beta}+\frac{2}{3} \sin \theta_{b} \tan \theta_{W} N_{i 1}\right) \operatorname{sign}\left(m_{\tilde{\chi}_{i}^{0}}\right) \\
& C_{\tilde{b}_{2}-b-\tilde{\chi}_{i}^{0}}^{L}=\frac{-g_{2}}{\sqrt{2}}\left(\sin \theta_{b}\left(N_{i 2}-\frac{\tan \theta_{W}}{3} N_{i 1}\right)+\cos \theta_{b} \frac{m_{b} N_{i 3}}{m_{W} \cos \beta}\right) \\
& C_{\tilde{b}_{2}-b-\tilde{\chi}_{i}^{0}}^{R}=\frac{-g_{2}}{\sqrt{2}}\left(-\sin \theta_{b} \frac{m_{b} N_{i 3}}{m_{W} \cos \beta}+\frac{2}{3} \cos \theta_{b} \tan \theta_{W} N_{i 1}\right) \operatorname{sign}\left(m_{\tilde{\chi}_{i}^{0}}\right) \\
& C_{\tilde{\ell}_{1}-\ell-\tilde{\chi}_{i}^{0}}^{L}=\frac{-g_{2}}{\sqrt{2}}\left(-\cos \theta_{\ell}\left(N_{i 2}+\tan \theta_{W} N_{i 1}\right)+\sin \theta_{\ell} \frac{m_{\ell} N_{i 3}}{m_{W} \cos \beta}\right) \\
& C_{\tilde{\ell}_{1}-\ell-\tilde{\chi}_{i}^{0}}^{R}=\frac{-g_{2}}{\sqrt{2}}\left(\cos \theta_{\ell} \frac{m_{\ell} N_{i 3}}{m_{W} \cos \beta}+2 \sin \theta_{\ell} \tan \theta_{W} N_{i 1}\right) \operatorname{sign}\left(m_{\tilde{\chi}_{i}^{0}}\right) \\
& C_{\tilde{\chi}_{i}^{0}-\tilde{\chi}_{j}^{0}-h}^{L}=\frac{g_{2}}{2}\left[\left(N_{i 3}\left(N_{j 2}-N_{j 1} \tan \theta_{W}\right)+N_{j 3}\left(N_{i 2}-N_{i 1} \tan \theta_{W}\right)\right) \operatorname{sign}\left(m_{\tilde{\chi}_{i}^{0}}\right) \sin \alpha\right. \\
& \left.+\left(N_{i 4}\left(N_{j 2}-N_{j 1} \tan \theta_{W}\right)+N_{j 4}\left(N_{i 2}-N_{i 1} \tan \theta_{W}\right)\right) \cos \alpha\right] \\
& C_{\tilde{\chi}_{i}^{0}-\tilde{\chi}_{j}^{0}-h}^{R}=\frac{g_{2}}{2}\left[\left(N_{j 3}\left(N_{i 2}-N_{i 1} \tan \theta_{W}\right)+N_{i 3}\left(N_{j 2}-N_{j 1} \tan \theta_{W}\right)\right) \operatorname{sign}\left(m_{\tilde{\chi}_{j}^{0}}\right) \sin \alpha\right. \\
& \left.+\left(N_{j 4}\left(N_{i 2}-N_{i 1} \tan \theta_{W}\right)+N_{i 4}\left(N_{j 2}-N_{j 1} \tan \theta_{W}\right)\right) \cos \alpha\right] \\
& C_{\tilde{\chi}_{i}^{0}-\tilde{\chi}_{j}^{0}-H}^{L}=-\frac{g_{2}}{2}\left[\left(N_{i 3}\left(N_{j 2}-N_{j 1} \tan \theta_{W}\right)+N_{j 3}\left(N_{i 2}-N_{i 1} \tan \theta_{W}\right)\right) \operatorname{sign}\left(m_{\tilde{\chi}_{i}^{0}}\right)\right. \\
& \left.\times \cos \alpha-\left(N_{i 4}\left(N_{j 2}-N_{j 1} \tan \theta_{W}\right)+N_{j 4}\left(N_{i 2}-N_{i 1} \tan \theta_{W}\right)\right) \sin \alpha\right] \\
& C_{\tilde{\chi}_{i}^{0}-\tilde{\chi}_{j}^{0}-H}^{R}=-\frac{g_{2}}{2}\left[\left(N_{j 3}\left(N_{i 2}-N_{i 1} \tan \theta_{W}\right)+N_{i 3}\left(N_{j 2}-N_{j 1} \tan \theta_{W}\right)\right) \operatorname{sign}\left(m_{\tilde{\chi}_{j}^{0}}\right)\right. \\
& \left.\times \cos \alpha-\left(N_{j 4}\left(N_{i 2}-N_{i 1} \tan \theta_{W}\right)+N_{i 4}\left(N_{j 2}-N_{j 1} \tan \theta_{W}\right)\right) \sin \alpha\right] \\
& C_{\tilde{\chi}_{i}^{0}-\tilde{\chi}_{j}^{0}-A}^{L}=\frac{g_{2}}{2}\left[\left(N_{i 3}\left(N_{j 2}-N_{j 1} \tan \theta_{W}\right)+N_{j 3}\left(N_{i 2}-N_{i 1} \tan \theta_{W}\right)\right) \operatorname{sign}\left(m_{\tilde{\chi}_{i}^{0}}\right)\right. \\
& \left.\times \sin \beta-\left(N_{i 4}\left(N_{j 2}-N_{j 1} \tan \theta_{W}\right)+N_{j 4}\left(N_{i 2}-N_{i 1} \tan \theta_{W}\right)\right) \cos \beta\right] \\
& C_{\tilde{\chi}_{i}^{0}-\tilde{\chi}_{j}^{0}-A}^{R}=-\frac{g_{2}}{2}\left[\left(\left(N_{j 3}\left(N_{i 2}-N_{i 1} \tan \theta_{W}\right)+N_{i 3}\left(N_{j 2}-N_{j 1} \tan \theta_{W}\right)\right) \operatorname{sign}\left(m_{\tilde{\chi}_{j}^{0}}\right)\right)\right. \\
& \left.\times \sin \beta-\left(N_{j 4}\left(N_{i 2}-N_{i 1} \tan \theta_{W}\right)+N_{i 4}\left(N_{j 2}-N_{j 1} \tan \theta_{W}\right)\right) \cos \beta\right]
\end{aligned}
$$




$$
\begin{aligned}
C_{t-b-H^{+}}^{L} & =\frac{g_{2}}{\sqrt{2} m_{W}} m_{b} \tan \beta \\
C_{t-b-H^{+}}^{R} & =\frac{g_{2}}{\sqrt{2} m_{W}} m_{t} \cot \beta \\
C_{\tilde{\chi}_{i}^{0}-\tilde{\chi}_{j}^{-}-H^{+}}^{L} & =-g_{2}\left(N_{i 3} U_{j 1}-\left(N_{i 2}+N_{i 1} \tan \theta_{W}\right) U_{j 2} / \sqrt{2}\right) \operatorname{sign}\left(m_{\tilde{\chi}_{i}^{0}}\right) \sin \beta \\
C_{\tilde{\chi}_{i}^{0}-\tilde{\chi}_{j}^{-}-H^{+}}^{R} & =-g_{2}\left(N_{i 4} V_{j 1}+\left(N_{i 2}+N_{i 1} \tan \theta_{W}\right) V_{j 2} / \sqrt{2}\right) \cos \beta \\
C_{\tilde{\chi}_{i}^{0}-\tilde{\chi}_{j}^{0}-Z}^{L} & =\frac{g_{2}}{4 \cos \theta_{W}} \operatorname{sign}\left(m_{\tilde{\chi}_{j}^{0}}\right)\left(N_{i 4} N_{j 4}-N_{i 3} N_{j 3}\right) \\
C_{\tilde{\chi}_{i}^{0}-\tilde{\chi}_{j}^{0}-Z}^{R} & =-C_{\tilde{\chi}_{i}^{0}-\tilde{\chi}_{j}^{0}-Z}^{L} \\
C_{\tilde{\chi}_{i}^{0}-\tilde{\chi}_{j}^{-}-W^{+}}^{L} & =g_{2}\left(N_{i 2} V_{j 1}-N_{i 4} V_{j 2} / \sqrt{2}\right) \\
C_{\tilde{\chi}_{i}^{0}-\tilde{\chi}_{j}^{-}-W^{+}}^{R} & =g_{2}\left(N_{i 2} U_{j 1}+N_{i 3} U_{j 2} / \sqrt{2}\right)
\end{aligned}
$$

\section{Squared Amplitudes}

Below is the list of the amplitudes squared. Note that, for identical-particle final states, one needs to divide them by two when performing the momentum integrations.

$\tilde{t}_{1} \tilde{t}_{1}^{*} \longrightarrow g g$

I. s-channel gluon annihilation

II. t-channel $\tilde{t}_{1}$ exchange

III. u-channel $\tilde{t}_{1}$ exchange

IV . point interaction

$$
\begin{aligned}
f_{1} & =C_{\tilde{t}_{1}-\tilde{t}_{1}-g} C_{g-g-g} \\
f_{2} & =\left(C_{\tilde{t}_{1}-\tilde{t}_{1}-g}\right)^{2} \\
f_{3} & =\left(C_{\tilde{t}_{1}-\tilde{t}_{1}-g}\right)^{2} \\
f_{4} & =C_{\tilde{t}_{1}-\tilde{t}_{1}-g-g} \\
\mathcal{T}_{\mathrm{I}} \times \mathcal{T}_{\mathrm{I}} & =(12 / 9)\left(4\left(m_{\tilde{t}_{1}}^{2}-s\right)+(5 / 2)(u-t)^{2}\right) / s^{2} \\
\mathcal{T}_{\mathrm{II}} \times \mathcal{T}_{\mathrm{II}} & =(16 / 27)\left(2 m_{\tilde{t}_{1}}^{2}+2 t\right)^{2} /\left(t-m_{\tilde{t}_{1}}^{2}\right)^{2} \\
\mathcal{T}_{\mathrm{III}} \times \mathcal{T}_{\mathrm{III}} & =(16 / 27)\left(2 m_{\tilde{t}_{1}}^{2}+2 u\right)^{2} /\left(u-m_{\tilde{t}_{1}}^{2}\right)^{2} \\
\mathcal{T}_{\mathrm{IV}} \times \mathcal{T}_{\mathrm{IV}} & =(28 / 27)(4) \\
\mathcal{T}_{\mathrm{I}} \times \mathcal{T}_{\mathrm{II}} & =0
\end{aligned}
$$




$$
\begin{aligned}
\mathcal{T}_{\mathrm{I}} \times \mathcal{T}_{\text {III }}= & 0 \\
\mathcal{T}_{\mathrm{I}} \times \mathcal{T}_{\text {IV }}= & 0 \\
\mathcal{T}_{\text {II }} \times \mathcal{T}_{\text {III }}= & (-2 / 27)\left(4 m_{\tilde{t}_{1}}^{2}-s\right)^{2} /\left(\left(u-m_{\tilde{t}_{1}}^{2}\right)\left(t-m_{\tilde{t}_{1}}^{2}\right)\right) \\
\mathcal{T}_{\text {II }} \times \mathcal{T}_{\text {IV }}= & (-14 / 27)\left(6 m_{\tilde{t}_{1}}^{2}-2 u-(5 / 2) s\right) /\left(t-m_{\tilde{t}_{1}}^{2}\right) \\
\mathcal{T}_{\text {III }} \times \mathcal{T}_{\text {IV }}= & (-14 / 27)\left(6 m_{\tilde{t}_{1}}^{2}-2 t-(5 / 2) s\right) /\left(u-m_{\tilde{t}_{1}}^{2}\right) \\
|\mathcal{T}|^{2}= & f_{1}^{2} \mathcal{T}_{\mathrm{I}} \times \mathcal{T}_{\mathrm{I}}+f_{2}^{2} \mathcal{T}_{\text {II }} \times \mathcal{T}_{\text {II }}+f_{3}^{2} \mathcal{T}_{\text {III }} \times \mathcal{T}_{\text {III }}+f_{4}^{2} \mathcal{T}_{\text {IV }} \times \mathcal{T}_{\text {IV }}+2 f_{1} f_{2} \mathcal{T}_{\text {I }} \times \mathcal{T}_{\text {II }}+ \\
& 2 f_{1} f_{3} \mathcal{T}_{\mathrm{I}} \times \mathcal{T}_{\text {III }}+2 f_{1} f_{4} \mathcal{T}_{\mathrm{I}} \times \mathcal{T}_{\text {IV }}+2 f_{2} f_{3} \mathcal{T}_{\text {II }} \times \mathcal{T}_{\text {III }}+2 f_{2} f_{4} \mathcal{T}_{\text {II }} \times \mathcal{T}_{\text {IV }}+ \\
& 2 f_{3} f_{4} \mathcal{T}_{\text {III }} \times \mathcal{T}_{\text {IV }}
\end{aligned}
$$

$\tilde{t}_{1} \tilde{t}_{1}^{*} \longrightarrow \gamma g$

I. t-channel $\tilde{t}_{1}$ exchange

II. u-channel $\tilde{t}_{1}$ exchange

III. point interaction

$$
\begin{aligned}
f_{1}= & C_{\tilde{t}_{1}-\tilde{t}_{1}-g} C_{\tilde{t}_{1}-\tilde{t}_{1}-\gamma} \\
f_{2}= & C_{\tilde{t}_{1}-\tilde{t}_{1}-g} C_{\tilde{t}_{1}-\tilde{t}_{1}-\gamma} \\
f_{3}= & C_{\tilde{t}_{1}-\tilde{t}_{1}-\gamma-g} \\
\mathcal{T}_{\mathrm{I}} \times \mathcal{T}_{\mathrm{I}}= & (4 / 9)\left(2 m_{\tilde{t}_{1}}^{2}+2 t\right)^{2} /\left(t-m_{\tilde{t}_{1}}^{2}\right)^{2} \\
\mathcal{T}_{\text {II }} \times \mathcal{T}_{\text {II }}= & (4 / 9)\left(2 m_{\tilde{t}_{1}}^{2}+2 u\right)^{2} /\left(u-m_{\tilde{t}_{1}}^{2}\right)^{2} \\
\mathcal{T}_{\text {III }} \times \mathcal{T}_{\text {III }}= & (4 / 9)(4) \\
\mathcal{T}_{\text {I }} \times \mathcal{T}_{\text {II }}= & (4 / 9)\left(4 m_{\tilde{t}_{1}}^{2}-s\right)^{2} /\left(\left(t-m_{\tilde{t}_{1}}^{2}\right)\left(u-m_{\tilde{t}_{1}}^{2}\right)\right) \\
\mathcal{T}_{\mathrm{I}} \times \mathcal{T}_{\text {III }}= & (-4 / 9)\left(6 m_{\tilde{t}_{1}}^{2}-(5 / 2) s-2 u\right) /\left(t-m_{\tilde{t}_{1}}^{2}\right) \\
\mathcal{T}_{\text {II }} \times \mathcal{T}_{\text {III }}= & (-4 / 9)\left(6 m_{\tilde{t}_{1}}^{2}-(5 / 2) s-2 t\right) /\left(u-m_{\tilde{t}_{1}}^{2}\right) \\
|\mathcal{T}|^{2}= & f_{1}^{2} \mathcal{T}_{\mathrm{I}} \times \mathcal{T}_{\mathrm{I}}+f_{2}^{2} \mathcal{T}_{\text {II }} \times \mathcal{T}_{\text {II }}+f_{3}^{2} \mathcal{T}_{\text {III }} \times \mathcal{T}_{\text {III }}+2 f_{1} f_{2} \mathcal{T}_{\mathrm{I}} \times \mathcal{T}_{\text {II }}+2 f_{1} f_{3} \mathcal{T}_{\mathrm{I}} \times \mathcal{T}_{\text {III }}+ \\
& 2 f_{2} f_{3} \mathcal{T}_{\text {II }} \times \mathcal{T}_{\text {III }}
\end{aligned}
$$


$\tilde{t}_{1} \tilde{t}_{1}^{*} \longrightarrow Z g$

I. t-channel $\tilde{t}_{1}$ exchange

II. u-channel $\tilde{t}_{1}$ exchange

III. point interaction

$$
\begin{aligned}
f_{1}= & C_{\tilde{t}_{1}-\tilde{t}_{1}-Z} C_{\tilde{t}_{1}-\tilde{t}_{1}-g} \\
f_{2}= & C_{\tilde{t}_{1}-\tilde{t}_{1}-Z} C_{\tilde{t}_{1}-\tilde{t}_{1}-g} \\
f_{3}= & C_{\tilde{t}_{1}-\tilde{t}_{1}-Z-g} \\
\mathcal{T}_{\mathrm{I}} \times \mathcal{T}_{\mathrm{I}}= & (4 / 9)\left(2 m_{\tilde{t}_{1}}^{2}-m_{Z}^{2}+2 t-\left(m_{\tilde{t}_{1}}^{2}-t\right)^{2} / m_{Z}^{2}\right)\left(2 m_{\tilde{t}_{1}}^{2}+2 t\right) /\left(t-m_{\tilde{t}_{1}}^{2}\right)^{2} \\
\mathcal{T}_{\mathrm{II}} \times \mathcal{T}_{\mathrm{II}}= & (4 / 9)\left(2 m_{\tilde{t}_{1}}^{2}-m_{Z}^{2}+2 u-\left(u-m_{\tilde{t}_{1}}^{2}\right)^{2} / m_{Z}^{2}\right)\left(2 m_{\tilde{t}_{1}}^{2}+2 u\right) /\left(u-m_{\tilde{t}_{1}}^{2}\right)^{2} \\
\mathcal{T}_{\mathrm{III}} \times \mathcal{T}_{\mathrm{III}}= & 12 / 9 \\
\mathcal{T}_{\mathrm{I}} \times \mathcal{T}_{\mathrm{II}}= & (4 / 9)\left(4 m_{\tilde{t}_{1}}^{2}-s-\left(u-m_{\tilde{t}_{1}}^{2}\right)\left(m_{\tilde{t}_{1}}^{2}-t\right) / m_{Z}^{2}\right)\left(4 m_{\tilde{t}_{1}}^{2}-m_{Z}^{2}-s\right) \\
& \left./\left(t-m_{\tilde{t}_{1}}^{2}\right)\left(u-m_{\tilde{t}_{1}}^{2}\right)\right) \\
\mathcal{T}_{\mathrm{I}} \times \mathcal{T}_{\mathrm{III}}= & -(4 / 9)\left(6 m_{\tilde{t}_{1}}^{2}+(3 / 2) m_{Z}^{2}-2 u-(5 / 2) s\right. \\
& \left.-\left(m_{\tilde{t}_{1}}^{2}-t\right)\left((1 / 2) s-(3 / 2) m_{Z}^{2}-m_{\tilde{t}_{1}}^{2}+u\right) / m_{Z}^{2}\right) /\left(t-m_{\tilde{t}_{1}}^{2}\right) \\
\mathcal{T}_{\mathrm{II}} \times \mathcal{T}_{\mathrm{III}}= & -(4 / 9)\left(6 m_{\tilde{t}_{1}}^{2}+(3 / 2) m_{Z}^{2}-2 t-(5 / 2) s\right. \\
& \left.-\left(m_{\tilde{t}_{1}}^{2}-u\right)\left((1 / 2) s-(3 / 2) m_{Z}^{2}-m_{\tilde{t}_{1}}^{2}+t\right) / m_{Z}^{2}\right) /\left(u-m_{\tilde{t}_{1}}^{2}\right) \\
\mid \mathcal{T}^{2}= & f_{1}^{2} \mathcal{T}_{\mathrm{I}} \times \mathcal{T}_{\mathrm{I}}+f_{2}^{2} \mathcal{T}_{\mathrm{II}} \times \mathcal{T}_{\mathrm{II}}+f_{3}^{2} \mathcal{T}_{\mathrm{III}} \times \mathcal{T}_{\mathrm{III}}+2 f_{1} f_{2} \mathcal{T}_{\mathrm{I}} \times \mathcal{T}_{\mathrm{II}}+2 f_{1} f_{3} \mathcal{T}_{\mathrm{I}} \times \mathcal{T}_{\mathrm{III}}+ \\
& 2 f_{2} f_{3} \mathcal{T}_{\mathrm{II}} \times \mathcal{T}_{\mathrm{III}}
\end{aligned}
$$

$\tilde{t}_{1} \tilde{t}_{1}^{*} \longrightarrow t \bar{t}$

I. s-channel gluon annihilation

II. t-channel gluino exchange

III. s-channel $h$ annihilation

IV. s-channel $H$ annihilation

V. t-channel $\tilde{\chi}_{(1,2,3,4)}^{0}$ exchange

VI. s-channel $Z$ annihilation

VII. s-channel $\gamma$ annihilation 


$$
\begin{aligned}
& f_{1}=C_{\tilde{t}_{1}-\tilde{t}_{1}-g} C_{t-t-g} \\
& f_{2 L}=\left(C_{\tilde{t}_{1}-t-\tilde{g}}^{L}\right)^{2} \\
& f_{2 R}=\left(C_{\tilde{t}_{1}-t-\tilde{g}}^{R}\right)^{2} \\
& f_{3}=C_{\tilde{t}_{1}-\tilde{t}_{1}-h} C_{t-t-h} \\
& f_{4}=C_{\tilde{t}_{1}-\tilde{t}_{1}-H} C_{t-t-H} \\
& J_{i}=\left(C_{\tilde{t}_{1}-t-\tilde{\chi}_{i}^{0}}^{L}+C_{\tilde{t}_{1}-t-\tilde{\chi}_{i}^{0}}^{R}\right) / 2 \\
& K_{i}=\left(C_{\tilde{t}_{1}-t-\tilde{\chi}_{i}^{0}}^{L}-C_{\tilde{t}_{1}-t-\tilde{\chi}_{i}^{0}}^{R}\right) / 2 \\
& f_{6 c}=C_{\tilde{t}_{1}-\tilde{t}_{1}-Z}\left(C_{t-t-Z}^{R}+C_{t-t-Z}^{L}\right) / 2 \\
& f_{6 d}=C_{\tilde{t}_{1}-\tilde{t}_{1}-Z}\left(C_{t-t-Z}^{R}-C_{t-t-Z}^{L}\right) / 2 \\
& f_{7 c}=C_{\tilde{t}_{1}-\tilde{t}_{1}-\gamma} C_{t-t-\gamma} \\
& f_{7 d}=0 \\
& \mathcal{T}_{\mathrm{I}} \times \mathcal{T}_{\mathrm{I}}=(2 / 9)\left(2 s\left(s-4 m_{\tilde{t}_{1}}^{2}\right)-2(u-t)^{2}\right) / s^{2} \\
& \mathcal{T}_{\text {II }} \times \mathcal{T}_{\text {II }}=(2 / 9)\left(\left(f_{2 L}^{2}+f_{2 R}^{2}\right)\left(\left(2 m_{t}^{2}-s\right) t-\left(m_{\tilde{t}_{1}}^{2}-m_{t}^{2}-t\right)^{2}\right)-4 \sqrt{f_{2 L} f_{2 R}}\left(f_{2 L}+f_{2 R}\right)\right. \\
& \left.\times m_{t} m_{\tilde{g}}\left(m_{\tilde{t}_{1}}^{2}-m_{t}^{2}-t\right)+2 f_{2 L} f_{2 R}\left(m_{\tilde{g}}^{2}\left(s-4 m_{t}^{2}\right)-2 m_{t}^{2} t\right)\right) /\left(t-m_{\tilde{g}}^{2}\right)^{2} \\
& \mathcal{T}_{\text {III }} \times \mathcal{T}_{\text {III }}=\left(2\left(s-4 m_{t}^{2}\right)\right) /\left(s-m_{h}^{2}\right)^{2} \\
& \mathcal{T}_{\text {IV }} \times \mathcal{T}_{\text {IV }}=\left(2\left(s-4 m_{t}^{2}\right)\right) /\left(s-m_{H}^{2}\right)^{2} \\
& \mathcal{T}_{\mathrm{V}} \times \mathcal{T}_{\mathrm{V}}=2\left(-m_{\tilde{t}_{1}}^{4}\left(4 J_{i} J_{j} K_{i} K_{j}+J_{i}^{2}\left(J_{j}^{2}+K_{j}^{2}\right)+K_{i}^{2}\left(J_{j}^{2}+K_{j}^{2}\right)\right)+4 m_{\tilde{\chi}_{i}^{0}} m_{\tilde{\chi}_{j}^{0}}\left(J_{i}^{2}-K_{i}^{2}\right)\right. \\
& \times\left(J_{j}^{2}-K_{j}^{2}\right) m_{t}^{2}-2 m_{\tilde{t}_{1}}^{2}\left(4 J_{i} J_{j} K_{i} K_{j}+J_{i}^{2}\left(J_{j}^{2}+K_{j}^{2}\right)+K_{i}^{2}\left(J_{j}^{2}+K_{j}^{2}\right)\right) m_{t}^{2} \\
& +4 m_{\tilde{\chi}_{j}^{0}}\left(J_{i}^{2}+K_{i}^{2}\right)\left(J_{j}^{2}-K_{j}^{2}\right) m_{t}^{3}+4 m_{\tilde{\chi}_{i}^{0}}\left(J_{i}^{2}-K_{i}^{2}\right)\left(J_{j}^{2}+K_{j}^{2}\right) m_{t}^{3} \\
& +3\left(4 J_{i} J_{j} K_{i} K_{j}+J_{i}^{2}\left(J_{j}^{2}+K_{j}^{2}\right)+K_{i}^{2}\left(J_{j}^{2}+K_{j}^{2}\right)\right) m_{t}^{4}-m_{\tilde{\chi}_{i}^{0}} m_{\tilde{\chi}_{j}^{0}}\left(J_{i}^{2}-K_{i}^{2}\right) \\
& \times\left(J_{j}^{2}-K_{j}^{2}\right) s+m_{\tilde{t}_{1}}^{2}\left(4 J_{i} J_{j} K_{i} K_{j}+J_{i}^{2}\left(J_{j}^{2}+K_{j}^{2}\right)+K_{i}^{2}\left(J_{j}^{2}+K_{j}^{2}\right)\right) s \\
& -m_{\tilde{\chi}_{j}^{0}}\left(J_{i}^{2}+K_{i}^{2}\right)\left(J_{j}^{2}-K_{j}^{2}\right) m_{t} s-m_{\tilde{\chi}_{i}^{0}}\left(J_{i}^{2}-K_{i}^{2}\right)\left(J_{j}^{2}+K_{j}^{2}\right) m_{t} s \\
& -\left(4 J_{i} J_{j} K_{i} K_{j}+J_{i}^{2}\left(J_{j}^{2}+K_{j}^{2}\right)+K_{i}^{2}\left(J_{j}^{2}+K_{j}^{2}\right)\right) m_{t}^{2} s+m_{\tilde{t}_{1}}^{2}\left(4 J_{i} J_{j} K_{i} K_{j}\right. \\
& \left.+J_{i}^{2}\left(J_{j}^{2}+K_{j}^{2}\right)+K_{i}^{2}\left(J_{j}^{2}+K_{j}^{2}\right)\right) t+m_{\tilde{\chi}_{j}^{0}}\left(J_{i}^{2}+K_{i}^{2}\right)\left(J_{j}^{2}-K_{j}^{2}\right) m_{t} t \\
& +m_{\tilde{\chi}_{i}^{0}}\left(J_{i}^{2}-K_{i}^{2}\right)\left(J_{j}^{2}+K_{j}^{2}\right) m_{t} t+2\left(-4 J_{i} J_{j} K_{i} K_{j}+J_{i}^{2}\left(J_{j}^{2}+K_{j}^{2}\right)\right. \\
& \left.+K_{i}^{2}\left(J_{j}^{2}+K_{j}^{2}\right)\right) m_{t}^{2} t+\left(4 J_{i} J_{j} K_{i} K_{j}+J_{i}^{2}\left(J_{j}^{2}+K_{j}^{2}\right)+K_{i}^{2}\left(J_{j}^{2}+K_{j}^{2}\right)\right) m_{t}^{2} t
\end{aligned}
$$




$$
\begin{aligned}
& +m_{\tilde{t}_{1}}^{2}\left(4 J_{i} J_{j} K_{i} K_{j}+J_{i}^{2}\left(J_{j}^{2}+K_{j}^{2}\right)+K_{i}^{2}\left(J_{j}^{2}+K_{j}^{2}\right)\right) u-m_{\tilde{\chi}_{j}^{0}}\left(J_{i}^{2}+K_{i}^{2}\right) \\
& \times\left(J_{j}^{2}-K_{j}^{2}\right) m_{t} u-m_{\tilde{\chi}_{i}^{0}}\left(J_{i}^{2}-K_{i}^{2}\right)\left(J_{j}^{2}+K_{j}^{2}\right) m_{t} u-\left(4 J_{i} J_{j} K_{i} K_{j}\right. \\
& \left.+J_{i}^{2}\left(J_{j}^{2}+K_{j}^{2}\right)+K_{i}^{2}\left(J_{j}^{2}+K_{j}^{2}\right)\right) m_{t}^{2} u-\left(4 J_{i} J_{j} K_{i} K_{j}+J_{i}^{2}\left(J_{j}^{2}+K_{j}^{2}\right)\right. \\
& \left.\left.+K_{i}^{2}\left(J_{j}^{2}+K_{j}^{2}\right)\right) t u\right) /\left(\left(m_{\tilde{\chi}_{i}^{0}}^{2}-t\right)\left(-m_{\tilde{\chi}_{j}^{0}}^{2}+t\right)\right) \\
& \mathcal{T}_{\mathrm{VI}} \times \mathcal{T}_{\mathrm{VI}}=\left(2 \left(16 f_{6 d}^{2} m_{\tilde{t}_{1}}^{2} m_{t}^{2}-4 f_{6 c}^{2} m_{\tilde{t}_{1}}^{2} s-4 f_{6 d}^{2} m_{\tilde{t}_{1}}^{2} s-4 f_{6 d}^{2} m_{t}^{2} s+f_{6 c}^{2} s^{2}+f_{6 d}^{2} s^{2}-f_{6 c}^{2} t^{2}\right.\right. \\
& \left.\left.-f_{6 d}^{2} t^{2}+2 f_{6 c}^{2} t u+2 f_{6 d}^{2} t u-f_{6 c}^{2} u^{2}-f_{6 d}^{2} u^{2}\right)\right) /\left(m_{Z}^{2}-s\right)^{2} \\
& \mathcal{T}_{\mathrm{VII}} \times \mathcal{T}_{\mathrm{VII}}=\left(2 \left(16 f_{7 d}^{2} m_{\tilde{t}_{1}}^{2} m_{t}^{2}-4 f_{7 c}^{2} m_{\tilde{t}_{1}}^{2} s-4 f_{7 d}^{2} m_{\tilde{t}_{1}}^{2} s-4 f_{7 d}^{2} m_{t}^{2} s+f_{7 c}^{2} s^{2}+f_{7 d}^{2} s^{2}-f_{7 c}^{2} t^{2}\right.\right. \\
& \left.\left.-f_{7 d}^{2} t^{2}+2 f_{7 c}^{2} t u+2 f_{7 d}^{2} t u-f_{7 c}^{2} u^{2}-f_{7 d}^{2} u^{2}\right)\right) / s^{2} \\
& \mathcal{T}_{\mathrm{I}} \times \mathcal{T}_{\mathrm{II}}=(2 / 27)\left(\left(f_{2 L}+f_{2 R}\right)\left((t-u)\left(m_{\tilde{t}_{1}}^{2}-m_{t}^{2}-t\right)-(1 / 2) s\left(4 m_{\tilde{t}_{1}}^{2}-s+t-u\right)\right)\right. \\
& \left.+4 \sqrt{f_{2 L} f_{2 R}} m_{\tilde{g}} m_{t}(t-u)\right) /\left(s\left(t-m_{\tilde{g}}^{2}\right)\right) \\
& \mathcal{T}_{\mathrm{I}} \times \mathcal{T}_{\text {III }}=0 \\
& \mathcal{T}_{\mathrm{I}} \times \mathcal{T}_{\mathrm{IV}}=0 \\
& \mathcal{T}_{\mathrm{I}} \times \mathcal{T}_{\mathrm{V}}=0 \\
& \mathcal{T}_{\mathrm{I}} \times \mathcal{T}_{\mathrm{VI}}=0 \\
& \mathcal{T}_{\mathrm{I}} \times \mathcal{T}_{\mathrm{VII}}=0 \\
& \mathcal{T}_{\text {II }} \times \mathcal{T}_{\text {III }}=0 \\
& \mathcal{T}_{\text {II }} \times \mathcal{T}_{\text {IV }}=0 \\
& \mathcal{T}_{\mathrm{II}} \times \mathcal{T}_{\mathrm{V}}=0 \\
& \mathcal{T}_{\mathrm{II}} \times \mathcal{T}_{\mathrm{VI}}=0 \\
& \mathcal{T}_{\text {II }} \times \mathcal{T}_{\text {VII }}=0 \\
& \mathcal{T}_{\mathrm{III}} \times \mathcal{T}_{\mathrm{IV}}=2\left(s-4 m_{t}^{2}\right) /\left(\left(s-m_{h}^{2}\right)\left(s-m_{H}^{2}\right)\right) \\
& \mathcal{T}_{\mathrm{III}} \times \mathcal{T}_{\mathrm{V}}=2 f_{3}\left(\left(J_{i}^{2}+K_{i}^{2}\right)\left(-4 m_{t}^{3}+m_{t} s-m_{t} t+m_{t} u\right)+\left(J_{i}^{2}-K_{i}^{2}\right)\left(-4 m_{t}^{2} m_{\tilde{\chi}_{i}^{0}}+m_{\tilde{\chi}_{i}^{0}} s\right)\right) \\
& /\left(\left(m_{h}^{2}-s\right)\left(m_{\tilde{\chi}_{i}^{0}}^{2}-t\right)\right) \\
& \mathcal{T}_{\mathrm{III}} \times \mathcal{T}_{\mathrm{VI}}=\left(4\left(f_{3} f_{6 c} m_{t} t-f_{3} f_{6 c} m_{t} u\right)\right) /\left(\left(m_{h}^{2}-s\right)\left(-m_{Z}^{2}+s\right)\right) \\
& \mathcal{T}_{\text {III }} \times \mathcal{T}_{\text {VII }}=\left(4\left(f_{3} f_{7 c} m_{t} t-f_{3} f_{7 c} m_{t} u\right)\right) /\left(\left(m_{h}^{2}-s\right) s\right) \\
& \mathcal{T}_{\mathrm{IV}} \times \mathcal{T}_{\mathrm{V}}=2 f_{4}\left(\left(J_{i}^{2}+K_{i}^{2}\right)\left(-4 m_{t}^{3}+m_{t} s-m_{t} t+m_{t} u\right)+\left(J_{i}^{2}-K_{i}^{2}\right)\left(-4 m_{t}^{2} m_{\tilde{\chi}_{i}^{0}}+m_{\tilde{\chi}_{i}^{0}} s\right)\right) \\
& /\left(\left(m_{H}^{2}-s\right)\left(m_{\tilde{\chi}_{i}^{0}}^{2}-t\right)\right)
\end{aligned}
$$




$$
\begin{aligned}
& \mathcal{T}_{\mathrm{IV}} \times \mathcal{T}_{\mathrm{VI}}=\left(4\left(f_{4} f_{6 c} m_{t} t-f_{4} f_{6 c} m_{t} u\right)\right) /\left(\left(m_{H}^{2}-s\right)\left(-m_{Z}^{2}+s\right)\right) \\
& \mathcal{T}_{\mathrm{IV}} \times \mathcal{T}_{\mathrm{VII}}=\left(4\left(f_{4} f_{7 c} m_{t} t-f_{4} f_{7 c} m_{t} u\right)\right) /\left(\left(m_{H}^{2}-s\right) s\right) \\
& \mathcal{T}_{\mathrm{V}} \times \mathcal{T}_{\mathrm{VI}}=\left(-16 f_{6 d} K_{i} J_{i} m_{\tilde{t}_{1}}^{2} m_{t}^{2}-16 f_{6 d} J_{i} K_{i} m_{\tilde{t}_{1}}^{2} m_{t}^{2}-4 f_{6 c} J_{i}^{2} m_{\tilde{t}_{1}}^{2} s+4 f_{6 d} K_{i} J_{i} m_{\tilde{t}_{1}}^{2} s\right. \\
& +4 f_{6 d} J_{i} K_{i} m_{\tilde{t}_{1}}^{2} s-4 f_{6 c} K_{i}^{2} m_{\tilde{t}_{1}}^{2} s+4 f_{6 d} K_{i} J_{i} m_{t}^{2} s+4 f_{6 d} J_{i} K_{i} m_{t}^{2} s+f_{6 c} J_{i}^{2} s^{2} \\
& -f_{6 d} K_{i} J_{i} s^{2}-f_{6 d} J_{i} K_{i} s^{2}+f_{6 c} K_{i}^{2} s^{2}-4 f_{6 c} J_{i}^{2} m_{t}^{2} t-4 f_{6 c} K_{i}^{2} m_{t}^{2} t \\
& -4 f_{6 c} J_{i}^{2} m_{t} m_{\tilde{\chi}_{i}^{0}} t+4 f_{6 c} K_{i}^{2} m_{t} m_{\tilde{\chi}_{i}^{0}} t-f_{6 c} J_{i}^{2} t^{2}+f_{6 d} K_{i} J_{i} t^{2}+f_{6 d} J_{i} K_{i} t^{2} \\
& -f_{6 c} K_{i}^{2} t^{2}+4 f_{6 c} J_{i}^{2} m_{t}^{2} u+4 f_{6 c} K_{i}^{2} m_{t}^{2} u+4 f_{6 c} J_{i}^{2} m_{t} m_{\tilde{\chi}_{i}^{0}} u \\
& -4 f_{6 c} K_{i}^{2} m_{t} m_{\tilde{\chi}_{i}^{0}} u+2 f_{6 c} J_{i}^{2} t u-2 f_{6 d} K_{i} J_{i} t u-2 f_{6 d} J_{i} K_{i} t u+2 f_{6 c} K_{i}^{2} t u \\
& \left.-f_{6 c} J_{i}^{2} u^{2}+f_{6 d} K_{i} J_{i} u^{2}+f_{6 d} J_{i} K_{i} u^{2}-f_{6 c} K_{i}^{2} u^{2}\right) /\left(\left(m_{Z}^{2}-s\right)\left(m_{\tilde{\chi}_{i}^{0}}^{2}-t\right)\right) \\
& \mathcal{T}_{\mathrm{V}} \times \mathcal{T}_{\mathrm{VII}}=-\left(\left(-4 f_{7 c} J_{i}^{2} m_{\tilde{t}_{1}}^{2} s-4 f_{7 c} K_{i}^{2} m_{\tilde{t}_{1}}^{2} s+f_{7 c} J_{i}^{2} s^{2}+f_{7 c} K_{i}^{2} s^{2}-4 f_{7 c} J_{i}^{2} m_{t}^{2} t\right.\right. \\
& -4 f_{7 c} K_{i}^{2} m_{t}^{2} t-4 f_{7 c} J_{i}^{2} m_{t} m_{\tilde{\chi}_{i}^{0}} t+4 f_{7 c} K_{i}^{2} m_{t} m_{\tilde{\chi}_{i}^{0}} t-f_{7 c} J_{i}^{2} t^{2}-f_{7 c} K_{i}^{2} t^{2} \\
& +4 f_{7 c} J_{i}^{2} m_{t}^{2} u+4 f_{7 c} K_{i}^{2} m_{t}^{2} u+4 f_{7 c} J_{i}^{2} m_{t} m_{\tilde{\chi}_{i}^{0}} u-4 f_{7 c} K_{i}^{2} m_{t} m_{\tilde{\chi}_{i}^{0}} u \\
& \left.\left.+2 f_{7 c} J_{i}^{2} t u+2 f_{7 c} K_{i}^{2} t u-f_{7 c} J_{i}^{2} u^{2}-f_{7 c} K_{i}^{2} u^{2}\right) /\left(s\left(m_{\tilde{\chi}_{i}^{0}}^{2}-t\right)\right)\right) \\
& \mathcal{T}_{\mathrm{VI}} \times \mathcal{T}_{\mathrm{VII}}=\left(2 \left(-16 f_{6 d} f_{7 d} m_{\tilde{t}_{1}}^{2} m_{t}^{2}+4 f_{6 c} f_{7 c} m_{\tilde{t}_{1}}^{2} s+4 f_{6 d} f_{7 d} m_{\tilde{t}_{1}}^{2} s+4 f_{6 d} f_{7 d} m_{t}^{2} s\right.\right. \\
& -f_{6 c} f_{7 c} s^{2}-f_{6 d} f_{7 d} s^{2}+f_{6 c} f_{7 c} t^{2}+f_{6 d} f_{7 d} t^{2}-2 f_{6 c} f_{7 c} t u-2 f_{6 d} f_{7 d} t u \\
& \left.\left.+f_{6 c} f_{7 c} u^{2}+f_{6 d} f_{7 d} u^{2}\right)\right) /\left(\left(m_{Z}^{2}-s\right) s\right) \\
& |\mathcal{T}|^{2}=f_{1}^{2} \mathcal{T}_{\mathrm{I}} \times \mathcal{T}_{\mathrm{I}}+\mathcal{T}_{\mathrm{II}} \times \mathcal{T}_{\mathrm{II}}+f_{3}^{2} \mathcal{T}_{\mathrm{III}} \times \mathcal{T}_{\mathrm{III}}+f_{4}^{2} \mathcal{T}_{\mathrm{IV}} \times \mathcal{T}_{\mathrm{IV}}+\sum_{i, j=1}^{4} \mathcal{T}_{\mathrm{V}} \times \mathcal{T}_{\mathrm{V}}+\mathcal{T}_{\mathrm{VI}} \times \mathcal{T}_{\mathrm{VI}}+ \\
& \mathcal{T}_{\mathrm{VII}} \times \mathcal{T}_{\mathrm{VII}}+2 f_{1} \mathcal{T}_{\mathrm{I}} \times \mathcal{T}_{\mathrm{II}}+2 f_{1} f_{3} \mathcal{T}_{\mathrm{I}} \times \mathcal{T}_{\mathrm{III}}+2 f_{1} f_{4} \mathcal{T}_{\mathrm{I}} \times \mathcal{T}_{\mathrm{IV}}+2 f_{1} \sum_{i=1}^{4} \mathcal{T}_{\mathrm{I}} \times \mathcal{T}_{\mathrm{V}}+ \\
& 2 f_{1} \mathcal{T}_{\mathrm{I}} \times \mathcal{T}_{\mathrm{VI}}+2 f_{1} \mathcal{T}_{\mathrm{I}} \times \mathcal{T}_{\mathrm{VII}}+2 f_{3} \mathcal{T}_{\mathrm{II}} \times \mathcal{T}_{\mathrm{III}}+2 f_{4} \mathcal{T}_{\mathrm{II}} \times \mathcal{T}_{\mathrm{IV}}+2 \sum_{i=1}^{4} \mathcal{T}_{\mathrm{II}} \times \mathcal{T}_{\mathrm{V}}+ \\
& 2 \mathcal{T}_{\mathrm{II}} \times \mathcal{T}_{\mathrm{VI}}+2 \mathcal{T}_{\mathrm{II}} \times \mathcal{T}_{\mathrm{VII}}+2 f_{3} f_{4} \mathcal{T}_{\mathrm{III}} \times \mathcal{T}_{\mathrm{IV}}+2 f_{3} \sum_{i=1}^{4} \mathcal{T}_{\mathrm{III}} \times \mathcal{T}_{\mathrm{V}}+2 \mathcal{T}_{\mathrm{III}} \times \mathcal{T}_{\mathrm{VI}}+ \\
& 2 \mathcal{T}_{\text {III }} \times \mathcal{T}_{\text {VII }}+2 f_{4} \sum_{i=1}^{4} \mathcal{T}_{\text {IV }} \times \mathcal{T}_{\text {V }}+2 \mathcal{T}_{\text {IV }} \times \mathcal{T}_{\text {VI }}+2 \mathcal{T}_{\text {IV }} \times \mathcal{T}_{\text {VII }}+2 \mathcal{T}_{\text {V }} \times \mathcal{T}_{\text {VI }}+ \\
& 2 \mathcal{T}_{\mathrm{V}} \times \mathcal{T}_{\mathrm{VII}}+2 \mathcal{T}_{\mathrm{VI}} \times \mathcal{T}_{\mathrm{VII}}
\end{aligned}
$$


$\tilde{t}_{1} \tilde{t}_{1}^{*} \longrightarrow b \bar{b}$

I. s-channel $h$ annihilation

II. s-channel $H$ annihilation

III. t-channel $\tilde{\chi}_{(1,2)}^{+}$exchange

IV. s-channel gluon annihilation

$$
\begin{aligned}
& f_{1}=C_{\tilde{t}_{1}-\tilde{t}_{1}-h} C_{b-b-h} \\
& f_{2}=C_{\tilde{t}_{1}-\tilde{t}_{1}-H} C_{b-b-H} \\
& f_{3 L L}^{i}=C_{\tilde{t}_{1}-b-\tilde{\chi}_{i}^{+}}^{L} C_{\tilde{t}_{1}-b-\tilde{\chi}_{i}^{+}}^{L} \\
& f_{3 L R}^{i}=C_{\tilde{t}_{1}-b-\tilde{\chi}_{i}^{+}}^{L} C_{\tilde{t}_{1}-b-\tilde{\chi}_{i}^{+}}^{R} \\
& f_{3 R L}^{i}=C_{\tilde{t}_{1}-b-\tilde{\chi}_{i}^{+}}^{R} C_{\tilde{t}_{1}-b-\tilde{\chi}_{i}^{+}}^{L} \\
& f_{3 R R}^{i}=C_{\tilde{t}_{1}-b-\tilde{\chi}_{i}^{+}}^{R} C_{\tilde{t}_{1}-b-\tilde{\chi}_{i}^{+}}^{R} \\
& f_{4}=C_{\tilde{t}_{1}-\tilde{t}_{1}-g} C_{b-b-g} \\
& \mathcal{T}_{\mathrm{I}} \times \mathcal{T}_{\mathrm{I}}=2\left(s-4 m_{b}^{2}\right) /\left(s-m_{h}^{2}\right)^{2} \\
& \mathcal{T}_{\text {II }} \times \mathcal{T}_{\text {II }}=2\left(s-4 m_{b}^{2}\right) /\left(s-m_{H}^{2}\right)^{2} \\
& \mathcal{T}_{\text {III }} \times \mathcal{T}_{\text {III }}=\left(\left(f_{3 R R}^{i} f_{3 R R}^{j}+f_{3 L L}^{i} f_{3 L L}^{j}\right)\left(t\left(2 m_{b}^{2}-s\right)-\left(m_{\tilde{t}_{1}}^{2}-m_{b}^{2}-t\right)^{2}\right)+\left(f_{3 R L}^{i} f_{3 L R}^{j}\right.\right. \\
& \left.+f_{3 L R}^{i} f_{3 R L}^{j}\right) m_{\tilde{\chi}_{i}^{+}} m_{\tilde{\chi}_{j}^{+}}\left(s-2 m_{b}^{2}\right)-\left(f_{3 R R}^{i} f_{3 L R}^{j}+f_{3 L L}^{i} f_{3 R L}^{j}+f_{3 L L}^{i} f_{3 L R}^{j}+\right. \\
& \left.f_{3 R R}^{i} f_{3 R L}^{j}\right) m_{\tilde{\chi}_{j}^{+}} m_{b}\left(m_{\tilde{t}_{1}}^{2}-m_{b}^{2}-t\right)-\left(f_{3 R L}^{i} f_{3 R R}^{j}+f_{3 L R}^{i} f_{3 L L}^{j}+f_{3 L R}^{i} f_{3 R R}^{j}+\right. \\
& \left.f_{3 R L}^{i} f_{3 L L}^{j}\right) m_{\tilde{\chi}_{i}^{+}} m_{b}\left(m_{\tilde{t}_{1}}^{2}-m_{b}^{2}-t\right)-\left(f_{3 L L}^{i} f_{3 R R}^{j}+f_{3 R R}^{i} f_{3 L L}^{j}\right) m_{b}^{2} 2 t \\
& \left.-\left(f_{3 L R}^{i} f_{3 L R}^{j}+f_{3 R L}^{i} f_{3 R L}^{j}\right) 2 m_{b}^{2} m_{\tilde{\chi}_{i}^{+}} m_{\tilde{\chi}_{j}^{+}}\right) /\left(\left(t-m_{\tilde{\chi}_{i}^{+}}^{2}\right)\left(t-m_{\tilde{\chi}_{j}^{+}}^{2}\right)\right) \\
& \mathcal{T}_{\text {IV }} \times \mathcal{T}_{\text {IV }}=(2 / 9)\left(2 s\left(s-4 m_{\tilde{t}_{1}}^{2}\right)-2(u-t)^{2}\right) / s^{2} \\
& \mathcal{T}_{\mathrm{I}} \times \mathcal{T}_{\mathrm{II}}=2\left(s-4 m_{b}^{2}\right) /\left(\left(s-m_{H}^{2}\right)\left(s-m_{h}^{2}\right)\right) \\
& \mathcal{T}_{\mathrm{I}} \times \mathcal{T}_{\mathrm{III}}=\left(\left(f_{3 L L}^{i}+f_{3 R R}^{i}\right) 2 m_{b}\left(t+m_{b}^{2}-m_{\tilde{t}_{1}}^{2}\right)+\left(f_{3 R L}^{i}+f_{3 L R}^{i}\right) m_{\tilde{\chi}_{i}^{+}}\left(s-4 m_{b}^{2}\right)\right) \\
& /\left(\left(s-m_{h}^{2}\right)\left(t-m_{\tilde{\chi}_{i}^{+}}^{2}\right)\right) \\
& \mathcal{T}_{\mathrm{I}} \times \mathcal{T}_{\mathrm{IV}}=0 \\
& \mathcal{T}_{\text {II }} \times \mathcal{T}_{\text {III }}=\left(\left(f_{3 L L}^{i}+f_{3 R R}^{i}\right) 2 m_{b}\left(t+m_{b}^{2}-m_{\tilde{t}_{1}}^{2}\right)+\left(f_{3 R L}^{i}+f_{3 L R}^{i}\right) m_{\tilde{\chi}_{i}^{+}}\left(s-4 m_{b}^{2}\right)\right) \\
& /\left(\left(s-m_{H}^{2}\right)\left(t-m_{\tilde{\chi}_{i}^{+}}^{2}\right)\right) \\
& \mathcal{T}_{\text {II }} \times \mathcal{T}_{\text {IV }}=0
\end{aligned}
$$




$$
\begin{aligned}
\mathcal{T}_{\text {III }} \times \mathcal{T}_{\text {IV }}= & 0 \\
|\mathcal{T}|^{2}= & f_{1}^{2} \mathcal{T}_{\mathrm{I}} \times \mathcal{T}_{\mathrm{I}}+f_{2}^{2} \mathcal{T}_{\text {II }} \times \mathcal{T}_{\text {II }}+\sum_{i, j=1}^{2} \mathcal{T}_{\text {III }} \times \mathcal{T}_{\text {III }}+f_{4}^{2} \mathcal{T}_{\text {IV }} \times \mathcal{T}_{\text {IV }}+2 f_{1} f_{2} \mathcal{T}_{\mathrm{I}} \times \mathcal{T}_{\text {II }}+ \\
& 2 f_{1} \sum_{i=1}^{2} \mathcal{T}_{\mathrm{I}} \times \mathcal{T}_{\text {III }}+2 f_{1} f_{4} \mathcal{T}_{\text {I }} \times \mathcal{T}_{\text {IV }}+2 f_{2} \sum_{i=1}^{2} \mathcal{T}_{\text {II }} \times \mathcal{T}_{\text {III }}+2 f_{2} f_{4} \mathcal{T}_{\text {II }} \times \mathcal{T}_{\text {IV }}+ \\
& 2 f_{4} \sum_{i=1}^{2} \mathcal{T}_{\text {III }} \times \mathcal{T}_{\text {IV }}
\end{aligned}
$$

$\tilde{t}_{1} \tilde{t}_{1}^{*} \longrightarrow q \bar{q}, \quad q=u, d, s, c$

I. s-channel gluon annihilation

$$
\begin{aligned}
f_{1} & =C_{\tilde{t}_{1}-\tilde{t}_{1}-g} C_{q-q-g} \\
\mathcal{T}_{\mathrm{I}} \times \mathcal{T}_{\mathrm{I}} & =(2 / 9)\left(2 s\left(s-4 m_{\tilde{t}_{1}}^{2}\right)-2(u-t)^{2}\right) / s^{2} \\
|\mathcal{T}|^{2} & =f_{1}^{2} \mathcal{T}_{\mathrm{I}} \times \mathcal{T}_{\mathrm{I}}
\end{aligned}
$$

$\tilde{t}_{1} \tilde{t}_{1}^{*} \longrightarrow g H \quad[g h]$

I. t-channel $\tilde{t}_{1}$ exchange

II. u-channel $\tilde{t}_{1}$ exchange

$$
\begin{aligned}
& f_{1}=C_{\tilde{t}_{1}-\tilde{t}_{1}-g} C_{\tilde{t}_{1}-\tilde{t}_{1}-H} \quad\left[C_{\tilde{t}_{1}-\tilde{t}_{1}-g} C_{\tilde{t}_{1}-\tilde{t}_{1}-h}\right] \\
& f_{2}=C_{\tilde{t}_{1}-\tilde{t}_{1}-g} C_{\tilde{t}_{1}-\tilde{t}_{1}-H} \quad\left[C_{\tilde{t}_{1}-\tilde{t}_{1}-g} C_{\tilde{t}_{1}-\tilde{t}_{1}-h}\right] \\
& \mathcal{T}_{\mathrm{I}} \times \mathcal{T}_{\mathrm{I}}=(-4 / 9)\left(2\left(m_{\tilde{t}_{1}}^{2}+t\right)\right) /\left(t-m_{\tilde{t}_{1}}^{2}\right)^{2} \\
& \mathcal{T}_{\text {II }} \times \mathcal{T}_{\text {II }}=(-4 / 9)\left(2\left(m_{\tilde{t}_{1}}^{2}+u\right)\right) /\left(u-m_{\tilde{t}_{1}}^{2}\right)^{2} \\
& \mathcal{T}_{\mathrm{I}} \times \mathcal{T}_{\text {II }}=(-4 / 9)\left(6 m_{\tilde{t}_{1}}^{2}-u-t-2 s\right) /\left(\left(t-m_{\tilde{t}_{1}}^{2}\right)\left(u-m_{\tilde{t}_{1}}^{2}\right)\right) \\
& |\mathcal{T}|^{2}=f_{1}^{2} \mathcal{T}_{\mathrm{I}} \times \mathcal{T}_{\mathrm{I}}+f_{2}^{2} \mathcal{T}_{\text {II }} \times \mathcal{T}_{\text {II }}+2 f_{1} f_{2} \mathcal{T}_{\mathrm{I}} \times \mathcal{T}_{\text {II }}
\end{aligned}
$$

$\tilde{t}_{1} \tilde{t}_{1}^{*} \longrightarrow Z h \quad[Z H]$

I. t-channel $\tilde{t}_{(1,2)}$ exchange

II. u-channel $\tilde{t}_{(1,2)}$ exchange 


$$
\begin{aligned}
f_{1}^{i} & =C_{\tilde{t}_{1}-\tilde{t}_{i}-Z} C_{\tilde{t}_{1}-\tilde{t}_{i}-h} \\
f_{2}^{i} & =C_{\tilde{t}_{1}-\tilde{t}_{i}-Z} C_{\tilde{t}_{1}-\tilde{t}_{i}-h} \\
\mathcal{T}_{\mathrm{I}} \times \mathcal{T}_{\mathrm{I}} & =-\left(2 m_{\tilde{t}_{1}}^{2}+2 t-m_{Z}^{2}-\left(m_{\tilde{t}_{1}}^{2}-t\right)^{2} / m_{Z}^{2}\right) /\left(\left(t-m_{\tilde{t}_{i}}^{2}\right)\left(t-m_{\tilde{t}_{j}}^{2}\right)\right) \\
\mathcal{T}_{\mathrm{II}} \times \mathcal{T}_{\mathrm{II}} & =-\left(2 m_{\tilde{t}_{1}}^{2}+2 u-m_{Z}^{2}-\left(m_{\tilde{t}_{1}}^{2}-u\right)^{2} / m_{Z}^{2}\right) /\left(\left(u-m_{\tilde{t}_{i}}^{2}\right)\left(u-m_{\tilde{t}_{j}}^{2}\right)\right) \\
\mathcal{T}_{\mathrm{I}} \times \mathcal{T}_{\mathrm{II}} & =-\left(4 m_{\tilde{t}_{1}}^{2}-m_{h}^{2}-s-\left(m_{\tilde{t}_{1}}^{2}-t\right)\left(u-m_{\tilde{t}_{1}}^{2}\right) / m_{Z}^{2}\right) /\left(\left(t-m_{\tilde{t}_{i}}^{2}\right)\left(u-m_{\tilde{t}_{j}}^{2}\right)\right) \\
|\mathcal{T}|^{2} & =\sum_{i=1}^{2} \sum_{j=1}^{2}\left[f_{1}^{i} f_{1}^{j} \mathcal{T}_{\mathrm{I}} \times \mathcal{T}_{\mathrm{I}}+f_{2}^{i} f_{2}^{j} \mathcal{T}_{\mathrm{II}} \times \mathcal{T}_{\mathrm{II}}+2 f_{1}^{i} f_{2}^{j} \mathcal{T}_{\mathrm{I}} \times \mathcal{T}_{\mathrm{II}}\right]
\end{aligned}
$$

$\tilde{t}_{1} \tilde{t}_{1}^{*} \longrightarrow Z A$

I. s-channel $h$ annihilation

II. s-channel $H$ annihilation

III. t-channel $\tilde{t}_{2}$ exchange

IV. u-channel $\tilde{t}_{2}$ exchange

$$
\begin{aligned}
f_{1} & =C_{\tilde{t}_{1}-\tilde{t}_{1}-h} C_{h-Z-A} \\
f_{2} & =C_{\tilde{t}_{1}-\tilde{t}_{1}-H} C_{H-Z-A} \\
f_{3} & =C_{\tilde{t}_{1}-\tilde{t}_{2}-Z} C_{\tilde{t}_{1}-\tilde{t}_{2}-A} \\
f_{4} & =C_{\tilde{t}_{1}-\tilde{t}_{2}-Z} C_{\tilde{t}_{1}-\tilde{t}_{2}-A} \\
\mathcal{T}_{\mathrm{I}} \times \mathcal{T}_{\mathrm{I}} & =\left(\left(s-m_{A}^{2}\right)^{2} / m_{Z}^{2}-\left(2 s-m_{Z}^{2}+2 m_{A}^{2}\right)\right) /\left(s-m_{h}^{2}\right)^{2} \\
\mathcal{T}_{\mathrm{II}} \times \mathcal{T}_{\mathrm{II}} & =\left(\left(s-m_{A}^{2}\right)^{2} / m_{Z}^{2}-\left(2 s-m_{Z}^{2}+2 m_{A}^{2}\right)\right) /\left(s-m_{H}^{2}\right)^{2} \\
\mathcal{T}_{\mathrm{III}} \times \mathcal{T}_{\mathrm{III}} & =\left(\left(m_{\tilde{t}_{1}}^{2}-t\right)^{2} / m_{Z}^{2}-\left(2 m_{\tilde{t}_{1}}^{2}+2 t\right)\right) /\left(t-m_{\tilde{t}_{2}}^{2}\right)^{2} \\
\mathcal{T}_{\mathrm{IV}} \times \mathcal{T}_{\mathrm{IV}} & =\left(\left(m_{\tilde{t}_{1}}^{2}-u\right)^{2} / m_{Z}^{2}-\left(2 m_{\tilde{t}_{1}}^{2}+2 u\right)\right) /\left(u-m_{\tilde{t}_{2}}^{2}\right)^{2} \\
\mathcal{T}_{\mathrm{I}} \times \mathcal{T}_{\mathrm{II}} & =\left(\left(s-m_{A}^{2}\right)^{2} / m_{Z}^{2}-\left(2 s-m_{Z}^{2}+2 m_{A}^{2}\right)\right) /\left(\left(s-m_{H}^{2}\right)\left(s-m_{h}^{2}\right)\right) \\
\mathcal{T}_{\mathrm{I}} \times \mathcal{T}_{\mathrm{III}} & =\left(\left(s-m_{A}^{2}\right)\left(m_{\tilde{t}_{1}}^{2}-t\right) / m_{Z}^{2}-m_{\tilde{t}_{1}}^{2}+2 m_{A}^{2}-u\right) /\left(\left(s-m_{h}^{2}\right)\left(t-m_{\tilde{t}_{2}}^{2}\right)\right) \\
\mathcal{T}_{\mathrm{I}} \times \mathcal{T}_{\mathrm{IV}} & =\left(\left(s-m_{A}^{2}\right)\left(m_{\tilde{t}_{1}}^{2}-u\right) / m_{Z}^{2}-m_{\tilde{t}_{1}}^{2}+2 m_{A}^{2}-t\right) /\left(\left(s-m_{h}^{2}\right)\left(u-m_{\tilde{t}_{2}}^{2}\right)\right) \\
\mathcal{T}_{\mathrm{II}} \times \mathcal{T}_{\mathrm{III}} & =\left(\left(s-m_{A}^{2}\right)\left(m_{\tilde{t}_{1}}^{2}-t\right) / m_{Z}^{2}-m_{\tilde{t}_{1}}^{2}+2 m_{A}^{2}-u\right) /\left(\left(s-m_{H}^{2}\right)\left(t-m_{\tilde{t}_{2}}^{2}\right)\right) \\
\mathcal{T}_{\mathrm{II}} \times \mathcal{T}_{\mathrm{IV}} & =\left(\left(s-m_{A}^{2}\right)\left(m_{\tilde{t}_{1}}^{2}-u\right) / m_{Z}^{2}-m_{\tilde{t}_{1}}^{2}+2 m_{A}^{2}-t\right) /\left(\left(s-m_{H}^{2}\right)\left(u-m_{\tilde{t}_{2}}^{2}\right)\right)
\end{aligned}
$$




$$
\begin{aligned}
\mathcal{T}_{\text {III }} \times \mathcal{T}_{\text {IV }}= & \left(\left(u-m_{\tilde{t}_{1}}^{2}\right)\left(m_{\tilde{t}_{1}}^{2}-t\right) / m_{Z}^{2}-\left(4 m_{\tilde{t}_{1}}^{2}-m_{A}^{2}-s\right)\right) /\left(\left(t-m_{\tilde{t}_{2}}^{2}\right)\left(u-m_{\tilde{t}_{2}}^{2}\right)\right) \\
|\mathcal{T}|^{2}= & f_{1}^{2} \mathcal{T}_{\mathrm{I}} \times \mathcal{T}_{\mathrm{I}}+f_{2}^{2} \mathcal{T}_{\text {II }} \times \mathcal{T}_{\text {II }}+f_{3}^{2} \mathcal{T}_{\text {III }} \times \mathcal{T}_{\text {III }}+f_{4}^{2} \mathcal{T}_{\mathrm{IV}} \times \mathcal{T}_{\mathrm{IV}}+2 f_{1} f_{2} \mathcal{T}_{\mathrm{I}} \times \mathcal{T}_{\mathrm{II}}+ \\
& 2 f_{1} f_{3} \mathcal{T}_{\mathrm{I}} \times \mathcal{T}_{\mathrm{III}}+2 f_{1} f_{4} \mathcal{T}_{\mathrm{I}} \times \mathcal{T}_{\mathrm{IV}}+2 f_{2} f_{3} \mathcal{T}_{\mathrm{II}} \times \mathcal{T}_{\mathrm{III}}+2 f_{2} f_{4} \mathcal{T}_{\mathrm{II}} \times \mathcal{T}_{\mathrm{IV}}+ \\
& 2 f_{3} f_{4} \mathcal{T}_{\mathrm{III}} \times \mathcal{T}_{\mathrm{IV}}
\end{aligned}
$$

$$
\tilde{t}_{1} \tilde{t}_{1}^{*} \longrightarrow W^{+} H^{-} \quad\left[H^{+} W^{-}\right]
$$

I. s-channel $h$ annihilation

II. s-channel $H$ annihilation

III. t-channel $\tilde{b}_{(1,2)}$ exchange

$$
\begin{aligned}
f_{1}= & C_{\tilde{t}_{1}-\tilde{t}_{1}-h} C_{h-W^{+}-H^{-}} \\
f_{2}= & C_{\tilde{t}_{1}-\tilde{t}_{1}-H} C_{H-W^{+}-H^{-}} \\
f_{3}^{i}= & C_{\tilde{t}_{1}-\tilde{b}_{i}-W^{+}} C_{\tilde{t}_{1}-\tilde{b}_{i}-H^{+}} \\
\mathcal{T}_{\mathrm{I}} \times \mathcal{T}_{\mathrm{I}}= & \left(\left(s-m_{H^{+}}^{2}\right)^{2} / m_{W}^{2}-\left(2 s-m_{W}^{2}+2 m_{H^{+}}^{2}\right)\right) /\left(s-m_{h}^{2}\right)^{2} \\
\mathcal{T}_{\mathrm{II}} \times \mathcal{T}_{\mathrm{II}}= & \left(\left(s-m_{H^{+}}^{2}\right)^{2} / m_{W}^{2}-\left(2 s-m_{W}^{2}+2 m_{H^{+}}^{2}\right)\right) /\left(s-m_{H}^{2}\right)^{2} \\
\mathcal{T}_{\mathrm{III}} \times \mathcal{T}_{\mathrm{III}}= & \left(\left(m_{\tilde{t}_{1}}^{2}-t\right)^{2} / m_{W}^{2}-2\left(m_{\tilde{t}_{1}}^{2}+t\right)\right) /\left(\left(t-m_{\tilde{b}_{i}}^{2}\right)\left(t-m_{\tilde{b}_{j}}^{2}\right)\right) \\
\mathcal{T}_{\mathrm{I}} \times \mathcal{T}_{\mathrm{II}}= & \left(\left(s-m_{H^{+}}^{2}\right)^{2} / m_{W}^{2}-\left(2 s-m_{W}^{2}+2 m_{H^{+}}^{2}\right)\right) /\left(\left(s-m_{H}^{2}\right)\left(s-m_{h}^{2}\right)\right) \\
\mathcal{T}_{\mathrm{I}} \times \mathcal{T}_{\mathrm{III}}= & \left(\left(s-m_{H^{+}}^{2}\right)\left(m_{\tilde{t}_{1}}^{2}-t\right) / m_{W}^{2}-\left(m_{\tilde{t}_{1}}^{2}+2 m_{H^{+}}^{2}-u\right)\right) /\left(\left(s-m_{h}^{2}\right)\left(t-m_{\tilde{b}_{i}}^{2}\right)\right) \\
\mathcal{T}_{\mathrm{II}} \times \mathcal{T}_{\mathrm{III}}= & \left(\left(s-m_{H^{+}}^{2}\right)\left(m_{\tilde{t}_{1}}^{2}-t\right) / m_{W}^{2}-\left(m_{\tilde{t}_{1}}^{2}+2 m_{H^{+}}^{2}-u\right)\right) /\left(\left(s-m_{H}^{2}\right)\left(t-m_{\tilde{b}_{i}}^{2}\right)\right) \\
|\mathcal{T}|^{2}= & f_{1}^{2} \mathcal{T}_{\mathrm{I}} \times \mathcal{T}_{\mathrm{I}}+f_{2}^{2} \mathcal{T}_{\mathrm{II}} \times \mathcal{T}_{\mathrm{II}}+\sum_{i=1}^{2} \sum_{j=1}^{2} f_{3}^{i} f_{3}^{j} \mathcal{T}_{\mathrm{III}} \times \mathcal{T}_{\mathrm{III}}+2 f_{1} f_{2} \mathcal{T}_{\mathrm{I}} \times \mathcal{T}_{\mathrm{II}}+ \\
& 2 \sum_{i=1}^{2} f_{1} f_{3}^{i} \mathcal{T}_{\mathrm{I}} \times \mathcal{T}_{\text {III }}+2 \sum_{i=1}^{2} f_{2} f_{3}^{i} \mathcal{T}_{\mathrm{II}} \times \mathcal{T}_{\mathrm{III}}
\end{aligned}
$$

\section{$\tilde{t}_{1} \tilde{t}_{1}^{*} \longrightarrow H H \quad[h h] \quad[h H]$}

I. t-channel $\tilde{t}_{(1,2)}$ exchange

II. u-channel $\tilde{t}_{(1,2)}$ exchange

III. point interaction

IV. s-channel $h$ annihilation 
V. s-channel $H$ annihilation

$$
\begin{aligned}
& f_{1}^{i}=C_{\tilde{t}_{1}-\tilde{t}_{i}-H}^{2} \quad\left[C_{\tilde{t}_{1}-\tilde{t}_{i}-h}^{2}\right] \quad\left[C_{\tilde{t}_{1}-\tilde{t}_{i}-h} C_{\tilde{t}_{1}-\tilde{t}_{i}-H}\right] \\
& f_{2}^{i}=C_{\tilde{t}_{1}-\tilde{t}_{i}-H}^{2} \quad\left[C_{\tilde{t}_{1}-\tilde{t}_{i}-h}^{2}\right] \quad\left[C_{\tilde{t}_{1}-\tilde{t}_{i}-h} C_{\tilde{t}_{1}-\tilde{t}_{i}-H}\right] \\
& f_{3}=C_{\tilde{t}_{1}-\tilde{t}_{1}-H-H}\left[\begin{array}{lll}
C_{\tilde{t}_{1}}-\tilde{t}_{1}-h-h & {\left[C_{\tilde{t}_{1}-\tilde{t}_{1}-h-H}\right]}
\end{array}\right. \\
& f_{4}=C_{\tilde{t}_{1}-\tilde{t}_{1}-h} C_{h-H-H} \quad\left[C_{\tilde{t}_{1}-\tilde{t}_{1}-h} C_{h-h-h}\right] \quad\left[C_{\tilde{t}_{1}-\tilde{t}_{1}-h} C_{h-h-H}\right] \\
& f_{5}=C_{\tilde{t}_{1}-\tilde{t}_{1}-H} C_{H-H-H} \quad\left[C_{\tilde{t}_{1}-\tilde{t}_{1}-H} C_{h-h-H}\right] \quad\left[C_{\tilde{t}_{1}-\tilde{t}_{1}-H} C_{h-H-H}\right] \\
& \mathcal{T}_{\mathrm{I}} \times \mathcal{T}_{\mathrm{I}}=1 /\left(\left(t-m_{\tilde{t}_{i}}^{2}\right)\left(t-m_{\tilde{t}_{j}}^{2}\right)\right) \\
& \mathcal{T}_{\text {II }} \times \mathcal{T}_{\text {II }}=1 /\left(\left(u-m_{\tilde{t}_{i}}^{2}\right)\left(u-m_{\tilde{t}_{j}}^{2}\right)\right) \\
& \mathcal{T}_{\text {III }} \times \mathcal{T}_{\text {III }}=1 \\
& \mathcal{T}_{\text {IV }} \times \mathcal{T}_{\text {IV }}=1 /\left(s-m_{h}^{2}\right)^{2} \\
& \mathcal{T}_{\mathrm{V}} \times \mathcal{T}_{\mathrm{V}}=1 /\left(s-m_{H}^{2}\right)^{2} \\
& \mathcal{T}_{\mathrm{I}} \times \mathcal{T}_{\mathrm{II}}=1 /\left(\left(t-m_{\tilde{t}_{i}}^{2}\right)\left(u-m_{\tilde{t}_{j}}^{2}\right)\right) \\
& \mathcal{T}_{\mathrm{I}} \times \mathcal{T}_{\text {III }}=-1 /\left(t-m_{\tilde{t}_{i}}^{2}\right) \\
& \mathcal{T}_{\mathrm{I}} \times \mathcal{T}_{\mathrm{IV}}=1 /\left(\left(t-m_{\tilde{t}_{i}}^{2}\right)\left(s-m_{h}^{2}\right)\right) \\
& \mathcal{T}_{\mathrm{I}} \times \mathcal{T}_{\mathrm{V}}=1 /\left(\left(t-m_{\tilde{t}_{i}}^{2}\right)\left(s-m_{H}^{2}\right)\right) \\
& \mathcal{T}_{\text {II }} \times \mathcal{T}_{\text {III }}=-1 /\left(u-m_{\tilde{t}_{i}}^{2}\right) \\
& \mathcal{T}_{\text {II }} \times \mathcal{T}_{\text {IV }}=1 /\left(\left(u-m_{\tilde{t}_{i}}^{2}\right)\left(s-m_{h}^{2}\right)\right) \\
& \mathcal{T}_{\mathrm{II}} \times \mathcal{T}_{\mathrm{V}}=1 /\left(\left(u-m_{\tilde{t}_{i}}^{2}\right)\left(s-m_{H}^{2}\right)\right) \\
& \mathcal{T}_{\text {III }} \times \mathcal{T}_{\text {IV }}=-1 /\left(s-m_{h}^{2}\right) \\
& \mathcal{T}_{\text {III }} \times \mathcal{T}_{\mathrm{V}}=-1 /\left(s-m_{H}^{2}\right) \\
& \mathcal{T}_{\mathrm{IV}} \times \mathcal{T}_{\mathrm{V}}=1 /\left(\left(s-m_{H}^{2}\right)\left(s-m_{h}^{2}\right)\right) \\
& |\mathcal{T}|^{2}=\sum_{i, j=1}^{2}\left(f_{1}^{i} f_{1}^{j} \mathcal{T}_{\mathrm{I}} \times \mathcal{T}_{\mathrm{I}}+f_{2}^{i} f_{2}^{j} \mathcal{T}_{\text {II }} \times \mathcal{T}_{\text {II }}+2 f_{1}^{i} f_{2}^{j} \mathcal{T}_{\mathrm{I}} \times \mathcal{T}_{\text {II }}\right)+2 \sum_{i=1}^{2}\left(f_{1}^{i} f_{3} \mathcal{T}_{\mathrm{I}} \times \mathcal{T}_{\text {III }}+\right. \\
& \left.f_{1}^{i} f_{4} \mathcal{T}_{\mathrm{I}} \times \mathcal{T}_{\mathrm{IV}}+f_{1}^{i} f_{5} \mathcal{T}_{\mathrm{I}} \times \mathcal{T}_{\mathrm{V}}+f_{2}^{i} f_{3} \mathcal{T}_{\mathrm{II}} \times \mathcal{T}_{\mathrm{III}}+f_{2}^{i} f_{4} \mathcal{T}_{\mathrm{II}} \times \mathcal{T}_{\mathrm{IV}}+f_{2}^{i} f_{5} \mathcal{T}_{\mathrm{II}} \times \mathcal{T}_{\mathrm{V}}\right)+ \\
& f_{3}^{2} \mathcal{T}_{\text {III }} \times \mathcal{T}_{\text {III }}+f_{4}^{2} \mathcal{T}_{\text {IV }} \times \mathcal{T}_{\text {IV }}+f_{5}^{2} \mathcal{T}_{\text {V }} \times \mathcal{T}_{\text {V }}+2 f_{3} f_{4} \mathcal{T}_{\text {III }} \times \mathcal{T}_{\text {IV }}+ \\
& 2 f_{3} f_{5} \mathcal{T}_{\mathrm{III}} \times \mathcal{T}_{\mathrm{V}}+2 f_{4} f_{5} \mathcal{T}_{\mathrm{IV}} \times \mathcal{T}_{\mathrm{V}}
\end{aligned}
$$


$\tilde{t}_{1} \tilde{t}_{1}^{*} \longrightarrow A A$

I. t-channel $\tilde{t}_{2}$ exchange

II. u-channel $\tilde{t}_{2}$ exchange

III. point interaction

IV. s-channel $h$ annihilation

V. s-channel $H$ annihilation

$$
\begin{aligned}
f_{1} & =C_{\tilde{t}_{1}-\tilde{t}_{2}-A}^{2} \\
f_{2} & =C_{\tilde{t}_{1}-\tilde{t}_{2}-A}^{2} \\
f_{3} & =C_{\tilde{t}_{1}-\tilde{t}_{1}-A-A} \\
f_{4} & =C_{\tilde{t}_{1}-\tilde{t}_{1}-h} C_{h-A-A} \\
f_{5} & =C_{\tilde{t}_{1}-\tilde{t}_{1}-H} C_{H-A-A} \\
\mathcal{T}_{\mathrm{I}} \times \mathcal{T}_{\mathrm{I}} & =1 /\left(t-m_{\tilde{t}_{2}}^{2}\right)^{2} \\
\mathcal{T}_{\mathrm{II}} \times \mathcal{T}_{\mathrm{II}} & =1 /\left(u-m_{\tilde{t}_{2}}^{2}\right)^{2} \\
\mathcal{T}_{\mathrm{III}} \times \mathcal{T}_{\mathrm{III}} & =1 \\
\mathcal{T}_{\mathrm{IV}} \times \mathcal{T}_{\mathrm{IV}} & =1 /\left(s-m_{h}^{2}\right)^{2} \\
\mathcal{T}_{\mathrm{V}} \times \mathcal{T}_{\mathrm{V}} & =1 /\left(s-m_{H}^{2}\right)^{2} \\
\mathcal{T}_{\mathrm{I}} \times \mathcal{T}_{\mathrm{II}} & =1 /\left(\left(t-m_{\tilde{t}_{2}}^{2}\right)\left(u-m_{\tilde{t}_{2}}^{2}\right)\right) \\
\mathcal{T}_{\mathrm{I}} \times \mathcal{T}_{\mathrm{III}} & =1 /\left(t-m_{\tilde{t}_{2}}^{2}\right) \\
\mathcal{T}_{\mathrm{I}} \times \mathcal{T}_{\mathrm{IV}} & =-1 /\left(\left(t-m_{\tilde{t}_{2}}^{2}\right)\left(s-m_{h}^{2}\right)\right) \\
\mathcal{T}_{\mathrm{I}} \times \mathcal{T}_{\mathrm{V}} & =-1 /\left(\left(t-m_{\tilde{t}_{2}}^{2}\right)\left(s-m_{H}^{2}\right)\right) \\
\mathcal{T}_{\mathrm{II}} \times \mathcal{T}_{\mathrm{III}} & =1 /\left(u-m_{\tilde{t}_{2}}^{2}\right) \\
\mathcal{T}_{\mathrm{II}} \times \mathcal{T}_{\mathrm{IV}} & =-1 /\left(\left(u-m_{\tilde{t}_{2}}^{2}\right)\left(s-m_{h}^{2}\right)\right) \\
\mathcal{T}_{\mathrm{II}} \times \mathcal{T}_{\mathrm{V}} & =-1 /\left(\left(t-m_{\tilde{t}_{2}}^{2}\right)\left(s-m_{H}^{2}\right)\right) \\
\mathcal{T}_{\mathrm{III}} \times \mathcal{T}_{\mathrm{IV}} & =-1 /\left(s-m_{h}^{2}\right) \\
\mathcal{T}_{\mathrm{III}} \times \mathcal{T}_{\mathrm{V}} & =-1 /\left(s-m_{H}^{2}\right) \\
\mathcal{T}_{\mathrm{IV}} \times \mathcal{T}_{\mathrm{V}} & =1 /\left(\left(s-m_{h}^{2}\right)\left(s-m_{H}^{2}\right)\right) \\
|\mathcal{T}|^{2} & =f_{1}^{2} \mathcal{T}_{\mathrm{I}} \times \mathcal{T}_{\mathrm{I}}+f_{2}^{2} \mathcal{T}_{\mathrm{II}} \times \mathcal{T}_{\mathrm{II}}+f_{3}^{2} \mathcal{T}_{\mathrm{III}} \times \mathcal{T}_{\mathrm{III}}+f_{4}^{2} \mathcal{T}_{\mathrm{IV}} \times \mathcal{T}_{\mathrm{IV}}+f_{5}^{2} \mathcal{T}_{\mathrm{V}} \times \mathcal{T}_{\mathrm{V}}+
\end{aligned}
$$




$$
\begin{aligned}
& 2 f_{1} f_{2} \mathcal{T}_{\mathrm{I}} \times \mathcal{T}_{\text {II }}+2 f_{1} f_{3} \mathcal{T}_{\mathrm{I}} \times \mathcal{T}_{\text {III }}+2 f_{1} f_{4} \mathcal{T}_{\mathrm{I}} \times \mathcal{T}_{\mathrm{IV}}+2 f_{1} f_{5} \mathcal{T}_{\mathrm{I}} \times \mathcal{T}_{\mathrm{V}}+ \\
& 2 f_{2} f_{3} \mathcal{T}_{\text {II }} \times \mathcal{T}_{\text {III }}+2 f_{2} f_{4} \mathcal{T}_{\text {II }} \times \mathcal{T}_{\mathrm{IV}}+2 f_{2} f_{5} \mathcal{T}_{\text {II }} \times \mathcal{T}_{\mathrm{V}}+2 f_{3} f_{4} \mathcal{T}_{\mathrm{III}} \times \mathcal{T}_{\mathrm{IV}}+ \\
& 2 f_{3} f_{5} \mathcal{T}_{\text {III }} \times \mathcal{T}_{\mathrm{V}}+2 f_{4} f_{5} \mathcal{T}_{\text {IV }} \times \mathcal{T}_{\mathrm{V}}
\end{aligned}
$$

$\tilde{t}_{1} \tilde{t}_{1}^{*} \longrightarrow h A \quad[H A]$

I. t-channel $\tilde{t}_{2}$ exchange

II. u-channel $\tilde{t}_{2}$ exchange

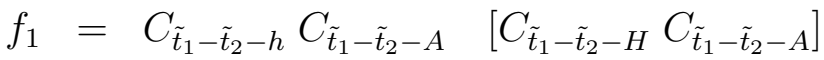

$$
\begin{aligned}
& f_{2}=C_{\tilde{t}_{1}-\tilde{t}_{2}-h} C_{\tilde{t}_{1}-\tilde{t}_{2}-A} \quad\left[\begin{array}{ll}
C_{\tilde{t}_{1}-\tilde{t}_{2}-H} & \left.C_{\tilde{t}_{1}-\tilde{t}_{2}-A}\right]
\end{array}\right. \\
& \mathcal{T}_{\mathrm{I}} \times \mathcal{T}_{\mathrm{I}}=1 /\left(t-m_{\tilde{t}_{2}}^{2}\right)^{2} \\
& \mathcal{T}_{\text {II }} \times \mathcal{T}_{\text {II }}=1 /\left(u-m_{\tilde{t}_{2}}^{2}\right)^{2} \\
& \mathcal{T}_{\mathrm{I}} \times \mathcal{T}_{\text {II }}=1 /\left(\left(t-m_{\tilde{t}_{2}}^{2}\right)\left(u-m_{\tilde{t}_{2}}^{2}\right)\right) \\
& |\mathcal{T}|^{2}=f_{1}^{2} \mathcal{T}_{\mathrm{I}} \times \mathcal{T}_{\mathrm{I}}+f_{2}^{2} \mathcal{T}_{\text {II }} \times \mathcal{T}_{\text {II }}+2 f_{1} f_{2} \mathcal{T}_{\text {I }} \times \mathcal{T}_{\text {II }}
\end{aligned}
$$

$\tilde{t}_{1} \tilde{t}_{1}^{*} \longrightarrow H^{+} H^{-}$

I. t-channel $\tilde{b}_{(1,2)}$ exchange

II. point interaction

III. s-channel $h$

IV. s-channel $H$

$$
\begin{aligned}
f_{1}^{i} & =\left(C_{\tilde{t}_{1}-\tilde{b}_{i}-H^{+}}\right)^{2} \\
f_{2} & =C_{\tilde{t}_{1}-\tilde{t}_{1}-H^{+}-H^{-}} \\
f_{3} & =C_{\tilde{t}_{1}-\tilde{t}_{1}-h} C_{h-H^{+}-H^{-}} \\
f_{4} & =C_{\tilde{t}_{1}-\tilde{t}_{1}-H} C_{H-H^{+}-H^{-}} \\
\mathcal{T}_{\mathrm{I}} \times \mathcal{T}_{\mathrm{I}} & =1 /\left(\left(t-m_{\tilde{b}_{i}}^{2}\right)\left(t-m_{\tilde{b}_{j}}^{2}\right)\right) \\
\mathcal{T}_{\mathrm{II}} \times \mathcal{T}_{\mathrm{II}} & =1 \\
\mathcal{T}_{\text {III }} \times \mathcal{T}_{\text {III }} & =1 /\left(s-m_{h}^{2}\right)^{2}
\end{aligned}
$$




$$
\begin{aligned}
& \mathcal{T}_{\text {IV }} \times \mathcal{T}_{\mathrm{IV}}=1 /\left(s-m_{H}^{2}\right)^{2} \\
& \mathcal{T}_{\mathrm{I}} \times \mathcal{T}_{\mathrm{II}}=-1 /\left(t-m_{\tilde{b}_{i}}^{2}\right) \\
& \mathcal{T}_{\mathrm{I}} \times \mathcal{T}_{\text {III }}=1 /\left(\left(s-m_{h}^{2}\right)\left(t-m_{\tilde{b}_{i}}^{2}\right)\right) \\
& \mathcal{T}_{\mathrm{I}} \times \mathcal{T}_{\mathrm{IV}}=1 /\left(\left(s-m_{H}^{2}\right)\left(t-m_{\tilde{b}_{i}}^{2}\right)\right) \\
& \mathcal{T}_{\text {II }} \times \mathcal{T}_{\text {III }}=-1 /\left(s-m_{h}^{2}\right) \\
& \mathcal{T}_{\text {II }} \times \mathcal{T}_{\text {IV }}=-1 /\left(s-m_{H}^{2}\right) \\
& \mathcal{T}_{\text {III }} \times \mathcal{T}_{\text {IV }}=1 /\left(\left(s-m_{h}^{2}\right)\left(s-m_{H}^{2}\right)\right) \\
& |\mathcal{T}|^{2}=\sum_{i, j=1}^{2} f_{1}^{i} f_{1}^{j} \mathcal{T}_{\mathrm{I}} \times \mathcal{T}_{\mathrm{I}}+f_{2}^{2} \mathcal{T}_{\text {II }} \times \mathcal{T}_{\text {II }}+f_{3}^{2} \mathcal{T}_{\text {III }} \times \mathcal{T}_{\text {III }}+f_{4}^{2} \mathcal{T}_{\text {IV }} \times \mathcal{T}_{\text {IV }}+2 \sum_{i=1}^{2} f_{1}^{i} f_{2} \mathcal{T}_{\mathrm{I}} \times \mathcal{T}_{\text {II }}+ \\
& 2 \sum_{i=1}^{2} f_{1}^{i} f_{3} \mathcal{T}_{\mathrm{I}} \times \mathcal{T}_{\mathrm{III}}+2 \sum_{i=1}^{2} f_{1}^{i} f_{4} \mathcal{T}_{\mathrm{I}} \times \mathcal{T}_{\mathrm{IV}}+2 f_{2} f_{3} \mathcal{T}_{\mathrm{II}} \times \mathcal{T}_{\mathrm{III}}+2 f_{2} f_{4} \mathcal{T}_{\mathrm{II}} \times \mathcal{T}_{\mathrm{IV}}+ \\
& 2 f_{3} f_{4} \mathcal{T}_{\text {III }} \times \mathcal{T}_{\text {IV }}
\end{aligned}
$$

$\tilde{t}_{1} \tilde{t}_{1} \longrightarrow t t$

I. t-channel gluino exchange

II. u-channel gluino exchange

III. t-channel $\chi_{(1,2,3,4)}^{0}$ exchange

IV. u-channel $\chi_{(1,2,3,4)}^{0}$ exchange

$$
\begin{aligned}
f_{1 L}= & f_{2 L} \equiv f_{L}=\left(C_{\tilde{t}_{1}-t-\tilde{g}}^{L}\right)^{2} \\
f_{1 R}= & f_{2 R} \equiv f_{R}=\left(C_{\tilde{t}_{1}-t-\tilde{g}}^{R}\right)^{2} \\
J_{i}= & \left(C_{\tilde{t}_{1}-t-\tilde{\chi}_{i}^{0}}^{L}+C_{\tilde{t}_{1}-t-\tilde{\chi}_{i}^{0}}^{R}\right) / 2 \\
K_{i}= & \left(C_{\tilde{t}_{1}-t-\tilde{\chi}_{i}^{0}}^{L}-C_{\tilde{t}_{1}-t-\tilde{\chi}_{i}^{0}}^{R}\right) / 2 \\
\mathcal{T}_{\mathrm{I}} \times \mathcal{T}_{\mathrm{I}}= & (2 / 9)\left(\left(f_{L}^{2}+f_{R}^{2}\right) m_{\tilde{g}}^{2}\left(s-2 m_{t}^{2}\right)-f_{L} f_{R}\left(4 m_{t}^{2} m_{\tilde{g}}^{2}+2 s t+2\left(m_{\tilde{t}_{1}}^{2}-m_{t}^{2}-t\right)^{2}\right)\right. \\
& \left.+\sqrt{f_{L} f_{R}}\left(f_{L}+f_{R}\right) 4 m_{t} m_{\tilde{g}}\left(t+m_{t}^{2}-m_{\tilde{t}_{1}}^{2}\right)\right) /\left(t-m_{\tilde{g}}^{2}\right)^{2} \\
\mathcal{T}_{\mathrm{II}} \times \mathcal{T}_{\mathrm{II}}= & (2 / 9)\left(\left(f_{L}^{2}+f_{R}^{2}\right) m_{\tilde{g}}^{2}\left(s-2 m_{t}^{2}\right)-f_{L} f_{R}\left(4 m_{t}^{2} m_{\tilde{g}}^{2}+2 s u+2\left(m_{\tilde{t}_{1}}^{2}-m_{t}^{2}-u\right)^{2}\right)\right. \\
& \left.+\sqrt{f_{L} f_{R}}\left(f_{L}+f_{R}\right) 4 m_{t} m_{\tilde{g}}\left(u+m_{t}^{2}-m_{\tilde{t}_{1}}^{2}\right)\right) /\left(u-m_{\tilde{g}}^{2}\right)^{2} \\
\mathcal{T}_{\mathrm{III}} \times \mathcal{T}_{\mathrm{III}}= & \left(-2\left(4 J_{i} J_{j} K_{i} K_{j} m_{\tilde{\chi}_{i}^{0}} m_{\tilde{\chi}_{j}^{0}} s+K_{i}^{2}\left(J _ { j } ^ { 2 } \left(-m_{\tilde{t}_{1}}^{4}-2 m_{\tilde{t}_{1}}^{2} m_{t}^{2}+3 m_{t}^{4}-4 m_{t}^{3} m_{\tilde{\chi}_{i}^{0}}\right.\right.\right.\right. \\
& +4 m_{t}^{3} m_{\tilde{\chi}_{j}^{0}}-4 m_{t}^{2} m_{\tilde{\chi}_{i}^{0}} m_{\tilde{\chi}_{j}^{0}}+m_{\tilde{t}_{1}}^{2} s-m_{t}^{2} s+m_{t} m_{\tilde{\chi}_{i}^{0}} s-m_{t} m_{\tilde{\chi}_{j}^{0}} s+m_{\tilde{\chi}_{i}^{0}} m_{\tilde{\chi}_{j}^{0}} s
\end{aligned}
$$




$$
\begin{aligned}
& +m_{\tilde{t}_{1}}^{2} t+3 m_{t}^{2} t-m_{t} m_{\tilde{\chi}_{i}^{0}} t+m_{t} m_{\tilde{\chi}_{j}^{0}} t+m_{\tilde{t}_{1}}^{2} u-m_{t}^{2} u+m_{t} m_{\tilde{\chi}_{i}^{0}} u-m_{t} m_{\tilde{\chi}_{j}^{0}} u \\
& -t u)+K_{j}^{2}\left(m_{\tilde{t}_{1}}^{4}+2 m_{\tilde{t}_{1}}^{2} m_{t}^{2}-3 m_{t}^{4}+4 m_{t}^{3} m_{\tilde{\chi}_{i}^{0}}+4 m_{t}^{3} m_{\tilde{\chi}_{j}^{0}}-4 m_{t}^{2} m_{\tilde{\chi}_{i}^{0}} m_{\tilde{\chi}_{j}^{0}}\right. \\
& -m_{\tilde{t}_{1}}^{2} s+m_{t}^{2} s-m_{t} m_{\tilde{\chi}_{i}^{0}} s-m_{t} m_{\tilde{\chi}_{j}^{0}} s+m_{\tilde{\chi}_{i}^{0}} m_{\tilde{\chi}_{j}^{0}} s-m_{\tilde{t}_{1}}^{2} t-3 m_{t}^{2} t+m_{t} m_{\tilde{\chi}_{i}^{0}} t \\
& \left.\left.+m_{t} m_{\tilde{\chi}_{j}^{0}} t-m_{\tilde{t}_{1}}^{2} u+m_{t}^{2} u-m_{t} m_{\tilde{\chi}_{i}^{0}} u-m_{t} m_{\tilde{\chi}_{j}^{0}} u+t u\right)\right)+J_{i}^{2}\left(K _ { j } ^ { 2 } \left(-m_{\tilde{t}_{1}}^{4}\right.\right. \\
& -2 m_{\tilde{t}_{1}}^{2} m_{t}^{2}+3 m_{t}^{4}+4 m_{t}^{3} m_{\tilde{\chi}_{i}^{0}}-4 m_{t}^{3} m_{\tilde{\chi}_{j}^{0}}-4 m_{t}^{2} m_{\tilde{\chi}_{i}^{0}} m_{\tilde{\chi}_{j}^{0}}+m_{\tilde{t}_{1}}^{2} s-m_{t}^{2} s \\
& -m_{t} m_{\tilde{\chi}_{i}^{0}} s+m_{t} m_{\tilde{\chi}_{j}^{0}} s+m_{\tilde{\chi}_{i}^{0}} m_{\tilde{\chi}_{j}^{0}} s+m_{\tilde{t}_{1}}^{2} t+3 m_{t}^{2} t+m_{t} m_{\tilde{\chi}_{i}^{0}} t-m_{t} m_{\tilde{\chi}_{j}^{0}} t \\
& \left.+m_{\tilde{t}_{1}}^{2} u-m_{t}^{2} u-m_{t} m_{\tilde{\chi}_{i}^{0}} u+m_{t} m_{\tilde{\chi}_{j}^{0}} u-t u\right)+J_{j}^{2}\left(m_{\tilde{t}_{1}}^{4}+2 m_{\tilde{t}_{1}}^{2} m_{t}^{2}-3 m_{t}^{4}\right. \\
& -4 m_{t}^{3} m_{\tilde{\chi}_{i}^{0}}-4 m_{t}^{3} m_{\tilde{\chi}_{j}^{0}}-4 m_{t}^{2} m_{\tilde{\chi}_{i}^{0}} m_{\tilde{\chi}_{j}^{0}}-m_{\tilde{t}_{1}}^{2} s+m_{t}^{2} s+m_{t} m_{\tilde{\chi}_{i}^{0}} s+m_{t} m_{\tilde{\chi}_{j}^{0}} s \\
& +m_{\tilde{\chi}_{i}^{0}} m_{\tilde{\chi}_{j}^{0}} s-m_{\tilde{t}_{1}}^{2} t-3 m_{t}^{2} t-m_{t} m_{\tilde{\chi}_{i}^{0}} t-m_{t} m_{\tilde{\chi}_{j}^{0}} t-m_{\tilde{t}_{1}}^{2} u+m_{t}^{2} u \\
& \left.\left.\left.\left.+m_{t} m_{\tilde{\chi}_{i}^{0}} u+m_{t} m_{\tilde{\chi}_{j}^{0}} u+t u\right)\right)\right)\right) /\left(\left(m_{\tilde{\chi}_{i}^{0}}^{2}-t\right)\left(-m_{\tilde{\chi}_{j}^{0}}^{2}+t\right)\right) \\
& \mathcal{T}_{\mathrm{IV}} \times \mathcal{T}_{\mathrm{IV}}=\left(-2\left(4 J_{i} J_{j} K_{i} K_{j} m_{\tilde{\chi}_{i}^{0}} m_{\tilde{\chi}_{j}^{0}} s+J_{i}^{2}\left(K _ { j } ^ { 2 } \left(-m_{\tilde{t}_{1}}^{4}-2 m_{\tilde{t}_{1}}^{2} m_{t}^{2}+3 m_{t}^{4}+4 m_{t}^{3} m_{\tilde{\chi}_{i}^{0}}\right.\right.\right.\right. \\
& -4 m_{t}^{3} m_{\tilde{\chi}_{j}^{0}}-4 m_{t}^{2} m_{\tilde{\chi}_{i}^{0}} m_{\tilde{\chi}_{j}^{0}}+m_{\tilde{t}_{1}}^{2} s-m_{t}^{2} s-m_{t} m_{\tilde{\chi}_{i}^{0}} s+m_{t} m_{\tilde{\chi}_{j}^{0}} s+m_{\tilde{\chi}_{i}^{0}} m_{\tilde{\chi}_{j}^{0}} s \\
& \left.+m_{\tilde{t}_{1}}^{2} t-m_{t}^{2} t-m_{t} m_{\tilde{\chi}_{i}^{0}} t+m_{t} m_{\tilde{\chi}_{j}^{0}} t+m_{\tilde{t}_{1}}^{2} u+3 m_{t}^{2} u+m_{t} m_{\tilde{\chi}_{i}^{0}} u-m_{t} m_{\tilde{\chi}_{j}^{0}} u-t u\right) \\
& +J_{j}^{2}\left(m_{\tilde{t}_{1}}^{4}+2 m_{\tilde{t}_{1}}^{2} m_{t}^{2}-3 m_{t}^{4}-4 m_{t}^{3} m_{\tilde{\chi}_{i}^{0}}-4 m_{t}^{3} m_{\tilde{\chi}_{j}^{0}}-4 m_{t}^{2} m_{\tilde{\chi}_{i}^{0}} m_{\tilde{\chi}_{j}^{0}}-m_{\tilde{t}_{1}}^{2} s\right. \\
& +m_{t}^{2} s+m_{t} m_{\tilde{\chi}_{i}^{0}} s+m_{t} m_{\tilde{\chi}_{j}^{0}} s+m_{\tilde{\chi}_{i}^{0}} m_{\tilde{\chi}_{j}^{0}} s-m_{\tilde{t}_{1}}^{2} t+m_{t}^{2} t+m_{t} m_{\tilde{\chi}_{i}^{0}} t+m_{t} m_{\tilde{\chi}_{j}^{0}} t \\
& \left.\left.-m_{\tilde{t}_{1}}^{2} u-3 m_{t}^{2} u-m_{t} m_{\tilde{\chi}_{i}^{0}} u-m_{t} m_{\tilde{\chi}_{j}^{0}} u+t u\right)\right)+K_{i}^{2}\left(J _ { j } ^ { 2 } \left(-m_{\tilde{t}_{1}}^{4}-2 m_{\tilde{t}_{1}}^{2} m_{t}^{2}\right.\right. \\
& +3 m_{t}^{4}-4 m_{t}^{3} m_{\tilde{\chi}_{i}^{0}}+4 m_{t}^{3} m_{\tilde{\chi}_{j}^{0}}-4 m_{t}^{2} m_{\tilde{\chi}_{i}^{0}} m_{\tilde{\chi}_{j}^{0}}+m_{\tilde{t}_{1}}^{2} s-m_{t}^{2} s+m_{t} m_{\tilde{\chi}_{i}^{0}} s \\
& -m_{t} m_{\tilde{\chi}_{j}^{0}} s+m_{\tilde{\chi}_{i}^{0}} m_{\tilde{\chi}_{j}^{0}} s+m_{\tilde{t}_{1}}^{2} t-m_{t}^{2} t+m_{t} m_{\tilde{\chi}_{i}^{0}} t-m_{t} m_{\tilde{\chi}_{j}^{0}} t+m_{\tilde{t}_{1}}^{2} u+3 m_{t}^{2} u \\
& \left.-m_{t} m_{\tilde{\chi}_{i}^{0}} u+m_{t} m_{\tilde{\chi}_{j}^{0}} u-t u\right)+K_{j}^{2}\left(m_{\tilde{t}_{1}}^{4}+2 m_{\tilde{t}_{1}}^{2} m_{t}^{2}-3 m_{t}^{4}+4 m_{t}^{3} m_{\tilde{\chi}_{i}^{0}}\right. \\
& +4 m_{t}^{3} m_{\tilde{\chi}_{j}^{0}}-4 m_{t}^{2} m_{\tilde{\chi}_{i}^{0}} m_{\tilde{\chi}_{j}^{0}}-m_{\tilde{t}_{1}}^{2} s+m_{t}^{2} s-m_{t} m_{\tilde{\chi}_{i}^{0}} s-m_{t} m_{\tilde{\chi}_{j}^{0}} s+m_{\tilde{\chi}_{i}^{0}} m_{\tilde{\chi}_{j}^{0}} s \\
& -m_{\tilde{t}_{1}}^{2} t+m_{t}^{2} t-m_{t} m_{\tilde{\chi}_{i}^{0}} t-m_{t} m_{\tilde{\chi}_{j}^{0}} t-m_{\tilde{t}_{1}}^{2} u-3 m_{t}^{2} u+m_{t} m_{\tilde{\chi}_{i}^{0}} u+m_{t} m_{\tilde{\chi}_{j}^{0}} u \\
& +t u)))) /\left(\left(m_{\tilde{\chi}_{i}^{0}}^{2}-u\right)\left(-m_{\tilde{\chi}_{j}^{0}}^{2}+u\right)\right) \\
& \mathcal{T}_{\mathrm{I}} \times \mathcal{T}_{\mathrm{II}}=(-2 / 27)\left(f_{L} f_{R}\left(2\left(m_{\tilde{t}_{1}}^{2}-m_{t}^{2}-u\right)\left(m_{\tilde{t}_{1}}^{2}-m_{t}^{2}-t\right)-4\left(s-2 m_{t}^{2}\right)\left(m_{\tilde{t}_{1}}^{2}-m_{t}^{2}\right)\right)\right. \\
& +\sqrt{f_{L} f_{R}}\left(f_{L}+f_{R}\right) m_{t} m_{\tilde{g}}(t-u)+\left(f_{L}^{2}+f_{R}^{2}\right) m_{\tilde{g}}^{2}\left(s-2 m_{t}^{2}\right)-4 m_{t}^{2} t f_{L} f_{R} \\
& \left.-m_{t} m_{\tilde{g}} \sqrt{f_{L} f_{R}}\left(f_{L}+f_{R}\right)(t+u)-4 m_{t}^{2} m_{\tilde{g}}^{2} f_{L} f_{R}\right) /\left(\left(t-m_{\tilde{g}}^{2}\right)\left(u-m_{\tilde{g}}^{2}\right)\right)
\end{aligned}
$$




$$
\begin{aligned}
& \mathcal{T}_{\text {II }} \times \mathcal{T}_{\text {III }}=0 \\
& \mathcal{T}_{\text {II }} \times \mathcal{T}_{\text {IV }}=0 \\
& \mathcal{T}_{\text {III }} \times \mathcal{T}_{\text {IV }}=\left(8 J_{i} J_{j} K_{i} K_{j} m_{\tilde{\chi}_{i}^{0}} m_{\tilde{\chi}_{j}^{0}} s+J_{i}^{2}\left(-\left(K _ { j } ^ { 2 } \left(2 m_{\tilde{t}_{1}}^{4}+4 m_{\tilde{t}_{1}}^{2} m_{t}^{2}-6 m_{t}^{4}-8 m_{t}^{3} m_{\tilde{\chi}_{i}^{0}}\right.\right.\right.\right. \\
& +8 m_{t}^{3} m_{\tilde{\chi}_{j}^{0}}+8 m_{t}^{2} m_{\tilde{\chi}_{i}^{0}} m_{\tilde{\chi}_{j}^{0}}+2 m_{\tilde{t}_{1}}^{2} s+2 m_{t}^{2} s+2 m_{t} m_{\tilde{\chi}_{i}^{0}} s-2 m_{t} m_{\tilde{\chi}_{j}^{0}} s \\
& -2 m_{\tilde{\chi}_{i}^{0}} m_{\tilde{\chi}_{j}^{0}} s-s^{2}-2 m_{\tilde{t}_{1}}^{2} t-2 m_{t}^{2} t+2 m_{t} m_{\tilde{\chi}_{i}^{0}} t+2 m_{t} m_{\tilde{\chi}_{j}^{0}} t+t^{2}-2 m_{\tilde{t}_{1}}^{2} u \\
& \left.\left.-2 m_{t}^{2} u-2 m_{t} m_{\tilde{\chi}_{i}^{0}} u-2 m_{t} m_{\tilde{\chi}_{j}^{0}} u+u^{2}\right)\right)+J_{j}^{2}\left(2 m_{\tilde{t}_{1}}^{4}+4 m_{\tilde{t}_{1}}^{2} m_{t}^{2}-6 m_{t}^{4}\right. \\
& -8 m_{t}^{3} m_{\tilde{\chi}_{i}^{0}}-8 m_{t}^{3} m_{\tilde{\chi}_{j}^{0}}-8 m_{t}^{2} m_{\tilde{\chi}_{i}^{0}} m_{\tilde{\chi}_{j}^{0}}+2 m_{\tilde{t}_{1}}^{2} s+2 m_{t}^{2} s+2 m_{t} m_{\tilde{\chi}_{i}^{0}} s+2 m_{t} m_{\tilde{\chi}_{j}^{0}} s \\
& +2 m_{\tilde{\chi}_{i}^{0}} m_{\tilde{\chi}_{j}^{0}} s-s^{2}-2 m_{\tilde{t}_{1}}^{2} t-2 m_{t}^{2} t+2 m_{t} m_{\tilde{\chi}_{i}^{0}} t-2 m_{t} m_{\tilde{\chi}_{j}^{0}} t+t^{2}-2 m_{\tilde{t}_{1}}^{2} u \\
& \left.\left.-2 m_{t}^{2} u-2 m_{t} m_{\tilde{\chi}_{i}^{0}} u+2 m_{t} m_{\tilde{\chi}_{j}^{0}} u+u^{2}\right)\right)+K_{i}^{2}\left(K _ { j } ^ { 2 } \left(2 m_{\tilde{t}_{1}}^{4}+4 m_{\tilde{t}_{1}}^{2} m_{t}^{2}-6 m_{t}^{4}\right.\right. \\
& +8 m_{t}^{3} m_{\tilde{\chi}_{i}^{0}}+8 m_{t}^{3} m_{\tilde{\chi}_{j}^{0}}-8 m_{t}^{2} m_{\tilde{\chi}_{i}^{0}} m_{\tilde{\chi}_{j}^{0}}+2 m_{\tilde{t}_{1}}^{2} s+2 m_{t}^{2} s-2 m_{t} m_{\tilde{\chi}_{i}^{0}} s-2 m_{t} m_{\tilde{\chi}_{j}^{0}} s \\
& +2 m_{\tilde{\chi}_{i}^{0}} m_{\tilde{\chi}_{j}^{0}} s-s^{2}-2 m_{\tilde{t}_{1}}^{2} t-2 m_{t}^{2} t-2 m_{t} m_{\tilde{\chi}_{i}^{0}} t+2 m_{t} m_{\tilde{\chi}_{j}^{0}} t+t^{2}-2 m_{\tilde{t}_{1}}^{2} u \\
& \left.-2 m_{t}^{2} u+2 m_{t} m_{\tilde{\chi}_{i}^{0}} u-2 m_{t} m_{\tilde{\chi}_{j}^{0}} u+u^{2}\right)-J_{j}^{2}\left(2 m_{\tilde{t}_{1}}^{4}+4 m_{\tilde{t}_{1}}^{2} m_{t}^{2}-6 m_{t}^{4}\right. \\
& +8 m_{t}^{3} m_{\tilde{\chi}_{i}^{0}}-8 m_{t}^{3} m_{\tilde{\chi}_{j}^{0}}+8 m_{t}^{2} m_{\tilde{\chi}_{i}^{0}} m_{\tilde{\chi}_{j}^{0}}+2 m_{\tilde{t}_{1}}^{2} s+2 m_{t}^{2} s-2 m_{t} m_{\tilde{\chi}_{i}^{0}} s+2 m_{t} m_{\tilde{\chi}_{j}^{0}} s \\
& -2 m_{\tilde{\chi}_{i}^{0}} m_{\tilde{\chi}_{j}^{0}} s-s^{2}-2 m_{\tilde{t}_{1}}^{2} t-2 m_{t}^{2} t-2 m_{t} m_{\tilde{\chi}_{i}^{0}} t-2 m_{t} m_{\tilde{\chi}_{j}^{0}} t+t^{2}-2 m_{\tilde{t}_{1}}^{2} u \\
& \left.\left.\left.-2 m_{t}^{2} u+2 m_{t} m_{\tilde{\chi}_{i}^{0}} u+2 m_{t} m_{\tilde{\chi}_{j}^{0}} u+u^{2}\right)\right)\right) /\left(\left(m_{\tilde{\chi}_{i}^{0}}^{2}-t\right)\left(m_{\tilde{\chi}_{j}^{0}}^{2}-u\right)\right) \\
& |\mathcal{T}|^{2}=\mathcal{T}_{\mathrm{I}} \times \mathcal{T}_{\mathrm{I}}+\mathcal{T}_{\mathrm{II}} \times \mathcal{T}_{\mathrm{II}}+\sum_{i, j=1}^{4}\left(\mathcal{T}_{\mathrm{III}} \times \mathcal{T}_{\mathrm{III}}+\mathcal{T}_{\mathrm{IV}} \times \mathcal{T}_{\mathrm{IV}}\right)+2 \mathcal{T}_{\mathrm{I}} \times \mathcal{T}_{\mathrm{II}}+ \\
& 2 \sum_{i=1}^{4}\left(\mathcal{T}_{\mathrm{I}} \times \mathcal{T}_{\mathrm{III}}+\mathcal{T}_{\mathrm{I}} \times \mathcal{T}_{\mathrm{IV}}+\mathcal{T}_{\mathrm{II}} \times \mathcal{T}_{\mathrm{III}}+\mathcal{T}_{\mathrm{II}} \times \mathcal{T}_{\mathrm{IV}}\right)+2 \sum_{i, j=1}^{4} \mathcal{T}_{\mathrm{III}} \times \mathcal{T}_{\mathrm{IV}}
\end{aligned}
$$

$\tilde{\chi} \tilde{t}_{1} \longrightarrow t g$

I. s-channel $t$ annihilation

II. t-channel $\tilde{t}_{1}$ exchange

$$
\begin{aligned}
f_{1 L} & =C_{\tilde{t}_{1}-t-\chi_{1}^{0}}^{L} C_{t-t-g} \\
f_{1 R} & =C_{\tilde{t}_{1}-t-\chi_{1}^{0}}^{R} C_{t-t-g} \\
f_{2 L} & =C_{\tilde{t}_{1}-t-\chi_{1}^{0}}^{L} C_{\tilde{t}_{1}-\tilde{t}_{1}-g} \\
f_{2 R} & =C_{\tilde{t}_{1}-t-\chi_{1}^{0}}^{R} C_{\tilde{t}_{1}-\tilde{t}_{1}-g}
\end{aligned}
$$




$$
\begin{aligned}
\mathcal{T}_{\mathrm{I}} \times \mathcal{T}_{\mathrm{I}}= & (-4 / 6)\left(\left(f_{1 L}^{2}+f_{1 R}^{2}\right) 2\left(\left(3 m_{t}^{2}-s\right)\left(s+m_{\tilde{\chi}}^{2}-m_{\tilde{t}_{1}}^{2}\right)+\left(s-m_{t}^{2}\right)\left(m_{\tilde{\chi}}^{2}+m_{t}^{2}-t\right)\right)\right. \\
& \left.-8 f_{1 L} f_{1 R} m_{t} m_{\tilde{\chi}}\left(s+m_{t}^{2}\right)\right) /\left(s-m_{t}^{2}\right)^{2} \\
\mathcal{T}_{\mathrm{II}} \times \mathcal{T}_{\mathrm{II}}= & (-4 / 6)\left(2\left(t+m_{\tilde{t}_{1}}^{2}\right)\left(\left(f_{2 L}^{2}+f_{2 R}^{2}\right)\left(m_{\tilde{\chi}}^{2}+m_{t}^{2}-t\right)-4 m_{\tilde{\chi}} m_{t} f_{2 L} f_{2 R}\right)\right) /\left(t-m_{\tilde{t}_{1}}^{2}\right)^{2} \\
\mathcal{T}_{\mathrm{I}} \times \mathcal{T}_{\mathrm{II}}= & (-4 / 6)\left(\left(f_{1 L} f_{2 L}+f_{1 R} f_{2 R}\right) / 2\left(\left(m_{\tilde{\chi}}^{2}+m_{t}^{2}-t\right)\left(s-2 m_{\tilde{\chi}}^{2}+2 m_{\tilde{t}_{1}}^{2}+m_{t}^{2}\right)\right.\right. \\
& -\left(s+m_{t}^{2}\right)\left(2 s-3 m_{\tilde{\chi}}^{2}-2 m_{\tilde{t}_{1}}^{2}+u\right)+\left(2 m_{\tilde{t}_{1}}^{2}+3 m_{t}^{2}-2 u-s\right)\left(s+m_{\tilde{\chi}}^{2}-m_{\tilde{t}_{1}}^{2}\right) \\
& \left.+2 m_{t}^{2}\left(2 s-3 m_{\tilde{\chi}}^{2}-2 m_{\tilde{t}_{1}}^{2}+u\right)\right)-\left(f_{1 L} f_{2 R}+f_{1 R} f_{2 L}\right) m_{t} m_{\tilde{\chi}}\left(4 m_{\tilde{t}_{1}}^{2}+4 m_{t}^{2}\right. \\
& \left.\left.-2 m_{\tilde{\chi}}^{2}-2 u\right)\right) /\left(\left(s-m_{t}^{2}\right)\left(t-m_{\tilde{t}_{1}}^{2}\right)\right) \\
|\mathcal{T}|^{2}= & \mathcal{T}_{\mathrm{I}} \times \mathcal{T}_{\mathrm{I}}+\mathcal{T}_{\mathrm{II}} \times \mathcal{T}_{\mathrm{II}}+2 \mathcal{T}_{\mathrm{I}} \times \mathcal{T}_{\mathrm{II}} \\
\tilde{\chi} \tilde{t}_{1} \longrightarrow t Z &
\end{aligned}
$$

I. u-channel $\tilde{\chi}_{(1,2,3,4)}^{0}$ exchange

$$
\begin{aligned}
f_{L L}^{i}= & C_{\tilde{t}_{1}-t-\tilde{\chi}_{i}^{0}}^{L} C_{\tilde{\chi}_{1}^{0}-\tilde{\chi}_{i}^{0}-Z}^{L} \\
f_{L R}^{i}= & C_{\tilde{t}_{1}-t-\tilde{\chi}_{i}^{0}}^{L} C_{\tilde{\chi}_{1}^{0}-\tilde{\chi}_{i}^{0}-Z}^{R} \\
f_{R L}^{i}= & C_{\tilde{t}_{1}-t-\tilde{\chi}_{i}^{0}}^{R} C_{\tilde{\chi}_{1}^{0}-\tilde{\chi}_{i}^{0}-Z}^{L} \\
f_{R R}^{i}= & C_{\tilde{t}_{1}-t-\tilde{\chi}_{i}^{0}}^{R} C_{\tilde{\chi}_{1}^{0}-\tilde{\chi}_{i}^{0}-Z}^{i} \\
\mathcal{T}_{\mathrm{I}} \times \mathcal{T}_{\mathrm{I}}= & (1 / 2)\left(( f _ { L L } ^ { i } f _ { L L } ^ { j } + f _ { R R } ^ { i } f _ { R R } ^ { j } ) \left(\left(\left(u-m_{\tilde{t}_{1}}^{2}+m_{t}^{2}\right)\left(u+m_{\tilde{\chi}}^{2}-m_{Z}^{2}\right)-\left(m_{\tilde{\chi}}^{2}+m_{t}^{2}-t\right) u\right)\right.\right. \\
& \left.+\left(\left(m_{\tilde{\chi}}^{2}+m_{Z}^{2}-u\right) / m_{Z}^{2}\right)\left(\left(m_{\tilde{\chi}}^{2}-m_{Z}^{2}-u\right)\left(u-m_{\tilde{t}_{1}}^{2}+m_{t}^{2}\right)-\left(s-m_{t}^{2}-m_{Z}^{2}\right) u\right)\right) \\
& +\left(f_{L R}^{i} f_{R R}^{j}+f_{R L}^{i} f_{L L}^{j}\right) m_{t} m_{\tilde{\chi}_{i}^{0}}\left(\left(u+m_{\tilde{\chi}}^{2}-m_{Z}^{2}\right)+\left(1 / m_{Z}^{2}\right)\left(\left(m_{\tilde{\chi}}^{2}+m_{Z}^{2}-u\right)\right.\right. \\
& \left.\left.\times\left(m_{\tilde{\chi}}^{2}-m_{Z}^{2}-u\right)\right)\right)+\left(f_{L L}^{i} f_{R L}^{j}+f_{R R}^{i} f_{L R}^{j}\right) m_{t} m_{\tilde{\chi}_{j}^{0}}\left(\left(u+m_{\tilde{\chi}}^{2}-m_{Z}^{2}\right)\right. \\
& \left.+\left(1 / m_{Z}^{2}\right)\left(\left(m_{\tilde{\chi}}^{2}+m_{Z}^{2}-u\right)\left(m_{\tilde{\chi}}^{2}-m_{Z}^{2}-u\right)\right)\right) \\
& +\left(f_{L R}^{i} f_{L R}^{j}+f_{R L}^{i} f_{R L}^{j}\right) m_{\tilde{\chi}_{i}^{0}} m_{\tilde{\chi}_{j}^{0}}\left(\left(t+m_{\tilde{\chi}}^{2}-m_{t}^{2}\right)\right. \\
& \left.+\left(1 / m_{Z}^{2}\right)\left(\left(m_{\tilde{\chi}}^{2}+m_{Z}^{2}-u\right)\left(s-m_{t}^{2}-m_{Z}^{2}\right)\right)\right) \\
& +\left(f_{L L}^{i} f_{R R}^{j}+f_{R R}^{i} f_{L L}^{j}\right) 6 m_{t} m_{\tilde{\chi}} u+\left(f_{L L}^{i} f_{L R}^{j}+f_{R R}^{i} f_{R L}^{j}\right) 3 m_{\tilde{\chi}_{j}^{0}}^{0}\left(u+m_{t}^{2}-m_{\tilde{t}_{1}}^{2}\right) m_{\tilde{\chi}} \\
& +\left(f_{L R}^{i} f_{L L}^{j}+f_{R L}^{i} f_{R R}^{j}\right) 3 m_{\tilde{\chi}_{i}^{0}}\left(u+m_{t}^{2}-m_{\tilde{t}_{1}}^{2}\right) m_{\tilde{\chi}} \\
& \left.+\left(f_{L R}^{i} f_{R L}^{j}+f_{R L}^{i} f_{L R}^{j}\right) 6 m_{\tilde{\chi}_{i}^{0}} m_{\tilde{\chi}_{j}^{0}} m_{t} m_{\tilde{\chi}}\right) /\left(\left(t-m_{\tilde{\chi}_{i}^{0}}^{2}\right)\left(t-m_{\tilde{\chi}_{j}^{0}}^{2}\right)\right) \\
|\mathcal{T}|^{2}= & \sum_{i, j=1}^{4} \mathcal{T}_{\mathrm{I}} \times \mathcal{T}_{\mathrm{I}}
\end{aligned}
$$


$\tilde{\chi} \tilde{t}_{1} \longrightarrow b W^{+}$

I. u-channel $\tilde{\chi}_{(1,2)}^{+}$exchange

$$
\begin{aligned}
f_{L L}^{i}= & C_{\tilde{t}_{1}-b-\tilde{\chi}_{i}^{+}}^{L} C_{\tilde{\chi}_{1}^{0}-\tilde{\chi}_{i}^{-}-W^{+}}^{L} \\
f_{L R}^{i}= & C_{\tilde{t}_{1}-b-\tilde{\chi}_{i}^{+}}^{L} C_{\tilde{\chi}_{1}^{0}-\tilde{\chi}_{i}^{-}-W^{+}} \\
f_{R L}^{i}= & C_{\tilde{t}_{1}-b-\tilde{\chi}_{i}^{+}}^{R} C_{\tilde{\chi}_{1}^{0}-\tilde{\chi}_{i}^{-}-W^{+}}^{L} \\
f_{R R}^{i}= & C_{\tilde{t}_{1}-t-\tilde{\chi}_{i}^{+}}^{R} C_{\tilde{\chi}_{1}^{0}-\tilde{\chi}_{i}^{-}-W^{+}} \\
\mathcal{T}_{\mathrm{I}} \times \mathcal{T}_{\mathrm{I}}= & (1 / 2)\left(( f _ { L L } ^ { i } f _ { L L } ^ { j } + f _ { R R } ^ { i } f _ { R R } ^ { j } ) \left(\left(\left(u-m_{\tilde{t}_{1}}^{2}+m_{b}^{2}\right)\left(u+m_{\tilde{\chi}}^{2}-m_{W}^{2}\right)-\left(m_{\tilde{\chi}}^{2}+m_{b}^{2}-t\right) u\right)\right.\right. \\
& \left.+\left(\left(m_{\tilde{\chi}}^{2}+m_{W}^{2}-u\right) / m_{W}^{2}\right)\left(\left(m_{\tilde{\chi}}^{2}-m_{W}^{2}-u\right)\left(u-m_{\tilde{t}_{1}}^{2}+m_{b}^{2}\right)-\left(s-m_{b}^{2}-m_{W}^{2}\right) u\right)\right) \\
& +\left(f_{L R}^{i} f_{R R}^{j}+f_{R L}^{i} f_{L L}^{j}\right) m_{b} m_{\tilde{\chi}_{i}^{+}}^{+}\left(\left(u+m_{\tilde{\chi}}^{2}-m_{W}^{2}\right)\right. \\
& \left.+\left(1 / m_{W}^{2}\right)\left(\left(m_{\tilde{\chi}}^{2}+m_{W}^{2}-u\right)\left(m_{\tilde{\chi}}^{2}-m_{W}^{2}-u\right)\right)\right) \\
& +\left(f_{L L}^{i} f_{R L}^{j}+f_{R R}^{i} f_{L R}^{j}\right) m_{b} m_{\tilde{\chi}_{j}^{+}}\left(\left(u+m_{\tilde{\chi}}^{2}-m_{W}^{2}\right)\right. \\
& \left.+\left(1 / m_{W}^{2}\right)\left(\left(m_{\tilde{\chi}}^{2}+m_{W}^{2}-u\right)\left(m_{\tilde{\chi}}^{2}-m_{W}^{2}-u\right)\right)\right) \\
& +\left(f_{L R}^{i} f_{L R}^{j}+f_{R L}^{i} f_{R L}^{j}\right) m_{\tilde{\chi}_{i}^{+}} m_{\tilde{\chi}_{j}^{+}}\left(\left(t+m_{\tilde{\chi}}^{2}-m_{b}^{2}\right)\right. \\
& \left.+\left(1 / m_{W}^{2}\right)\left(\left(m_{\tilde{\chi}}^{2}+m_{W}^{2}-u\right)\left(s-m_{b}^{2}-m_{W}^{2}\right)\right)\right) \\
& +\left(f_{L L}^{i} f_{R R}^{j}+f_{R R}^{i} f_{L L}^{j}\right) 6 m_{b} m_{\tilde{\chi}} u+\left(f_{L L}^{i} f_{L R}^{j}+f_{R R}^{i} f_{R L}^{j}\right) 3 m_{\tilde{\chi}_{j}^{+}}\left(u+m_{b}^{2}-m_{\tilde{t}_{1}}^{2}\right) m_{\tilde{\chi}} \\
& +\left(f_{L R}^{i} f_{L L}^{j}+f_{R L}^{i} f_{R R}^{j}\right) 3 m_{\tilde{\chi}_{i}^{+}}\left(u+m_{b}^{2}-m_{\tilde{t}_{1}}^{2}\right) m_{\tilde{\chi}} \\
& \left.+\left(f_{L R}^{i} f_{R L}^{j}+f_{R L}^{i} f_{L R}^{j}\right) 6 m_{\tilde{\chi}_{i}^{+}} m_{\tilde{\chi}_{j}^{+}} m_{b} m_{\tilde{\chi}}\right) /\left(\left(t-m_{\tilde{\chi}_{i}^{+}}^{2}\right)\left(t-m_{\tilde{\chi}_{j}^{+}}^{2}\right)\right) \\
|\mathcal{T}|^{2}= & \sum_{i, j=1}^{2} \mathcal{T}_{\mathrm{I} \times \mathcal{T}_{\mathrm{I}}} \\
\tilde{\chi} \tilde{t}_{1} \longrightarrow & t \quad[t h] \\
& {[\mathrm{A} 18) }
\end{aligned}
$$

I. s-channel $t$ annihilation

II. t-channel $\tilde{t}_{(1,2)}$ exchange

III. u-channel $\chi_{(1,2,3,4)}^{0}$ exchange

$$
\begin{aligned}
& f_{1 L}=C_{t_{1}-t-\tilde{\chi}_{1}^{0}}^{L} C_{t-t-H} \quad\left[C_{\tilde{t}_{1}-t-\tilde{\chi}_{1}^{0}}^{L} C_{t-t-h}\right] \\
& f_{1 R}=C_{\tilde{t}_{1}-t-\tilde{\chi}_{1}^{0}}^{R} C_{t-t-H} \quad\left[C_{\tilde{t}_{1}-t-\tilde{\chi}_{1}^{0}}^{R} C_{t-t-h}\right]
\end{aligned}
$$




$$
\begin{aligned}
& f_{2 L}^{i}=C_{\tilde{t}_{1}-t-\tilde{\chi}_{1}^{0}}^{L} C_{\tilde{t}_{1}-\tilde{t}_{1}-H} \quad\left[C_{\tilde{t}_{1}-t-\tilde{\chi}_{1}^{0}}^{L} C_{\tilde{t}_{1}-\tilde{t}_{1}-h}\right] \\
& f_{2 R}^{i}=C_{\tilde{t}_{1}-t-\tilde{\chi}_{1}^{0}}^{R} C_{\tilde{t}_{1}-\tilde{t}_{1}-H} \quad\left[C_{\tilde{t}_{1}-t-\tilde{\chi}_{1}^{0}}^{R} C_{\tilde{t}_{1}-\tilde{t}_{1}-h}\right] \\
& f_{3 L L}^{i}=C_{\tilde{t}_{1}-t-\tilde{\chi}_{i}^{0}}^{L} C_{\tilde{\chi}_{1}^{0}-\tilde{\chi}_{i}^{0}-H}^{L} \quad\left[C_{\tilde{t}_{1}-t-\tilde{\chi}_{i}^{0}}^{L} C_{\tilde{\chi}_{1}^{0}-\tilde{\chi}_{i}^{0}-h}^{L}\right] \\
& f_{3 L R}^{i}=C_{\tilde{t}_{1}-t-\tilde{\chi}_{i}^{0}}^{L} C_{\tilde{\chi}_{1}^{0}-\tilde{\chi}_{i}^{0}-H}^{R} \quad\left[C_{\tilde{t}_{1}-t-\tilde{\chi}_{i}^{0}}^{L} C_{\tilde{\chi}_{1}^{0}-\tilde{\chi}_{i}^{0}-h}^{R}\right] \\
& f_{3 R L}^{i}=C_{\tilde{t}_{1}-t-\tilde{\chi}_{i}^{0}}^{R} C_{\tilde{\chi}_{1}^{0}-\tilde{\chi}_{i}^{0}-H}^{L} \quad\left[C_{\tilde{t}_{1}-t-\tilde{\chi}_{i}^{0}}^{R} C_{\tilde{\chi}_{1}^{0}-\tilde{\chi}_{i}^{0}-h}^{L}\right] \\
& f_{3 R R}^{i}=C_{\tilde{t}_{1}-t-\tilde{\chi}_{i}^{0}}^{R} C_{\tilde{\chi}_{1}^{0}-\tilde{\chi}_{i}^{0}-H}^{R} \quad\left[C_{\tilde{t}_{1}-t-\tilde{\chi}_{i}^{0}}^{R} C_{\tilde{\chi}_{1}^{0}-\tilde{\chi}_{i}^{0}-h}^{R}\right] \\
& \mathcal{T}_{\mathrm{I}} \times \mathcal{T}_{\mathrm{I}}=(1 / 2)\left(( f _ { 1 L } ^ { 2 } + f _ { 1 R } ^ { 2 } ) \left(\left(s+m_{t}^{2}-m_{H[h]}^{2}\right)\left(s+m_{\tilde{\chi}}^{2}-m_{\tilde{t}_{1}}^{2}\right)-\left(s-m_{t}^{2}\right)\left(m_{\tilde{\chi}}^{2}+m_{t}^{2}\right.\right.\right. \\
& \left.\left.-t)+2 m_{t}^{2}\left(s+m_{\tilde{\chi}}^{2}-m_{\tilde{t}_{1}}^{2}\right)\right)-f_{1 L} f_{1 R} 4 m_{\tilde{\chi}} m_{t}\left(2 s+2 m_{t}^{2}-m_{H[h]}^{2}\right)\right) /\left(s-m_{t}^{2}\right)^{2} \\
& \mathcal{T}_{\text {II }} \times \mathcal{T}_{\text {II }}=(1 / 2)\left(\left(f_{2 L}^{i} f_{2 L}^{j}+f_{2 R}^{i} f_{2 R}^{j}\right)\left(m_{\tilde{\chi}}^{2}+m_{t}^{2}-t\right)-2 m_{t} m_{\tilde{\chi}}\left(f_{2 L}^{i} f_{2 R}^{j}+f_{2 R}^{i} f_{2 L}^{j}\right)\right) \\
& /\left(\left(t-m_{\tilde{t}_{i}}^{2}\right)\left(t-m_{\tilde{t}_{j}}^{2}\right)\right) \\
& \mathcal{T}_{\text {III }} \times \mathcal{T}_{\text {III }}=(1 / 2)\left(( f _ { 3 L R } ^ { i } f _ { 3 L R } ^ { j } + f _ { 3 R L } ^ { i } f _ { 3 R L } ^ { j } ) \left(\left(m_{t}^{2}-m_{\tilde{t}_{1}}^{2}+u\right)\left(m_{\tilde{\chi}}^{2}-m_{H[h]}^{2}+u\right)\right.\right. \\
& \left.-u\left(m_{\tilde{\chi}}^{2}+m_{t}^{2}-t\right)\right)+\left(f_{3 L L}^{i} f_{3 L L}^{j}+f_{3 R R}^{i} f_{3 R R}^{j}\right) m_{\tilde{\chi}_{i}^{0}} m_{\tilde{\chi}_{j}^{0}}\left(m_{\tilde{\chi}}^{2}+m_{t}^{2}-t\right) \\
& -\left(f_{3 L R}^{i} f_{3 L L}^{j}+f_{3 R L}^{i} f_{3 R R}^{j}\right) m_{\tilde{\chi}_{j}^{0}}\left(m_{t}^{2}-m_{\tilde{t}_{1}}^{2}+u\right)-\left(f_{3 L L}^{i} f_{3 L R}^{j}+f_{3 R R}^{i} f_{3 R L}^{j}\right) \\
& \times m_{\tilde{\chi}_{i}^{0}} m_{\tilde{\chi}}\left(m_{t}^{2}-m_{\tilde{t}_{1}}^{2}+u\right)+\left(f_{3 R L}^{i} f_{3 L L}^{j}+f_{3 L R}^{i} f_{3 R R}^{j}\right) m_{t} m_{\tilde{\chi}_{j}^{0}}\left(m_{\tilde{\chi}}^{2}-m_{H[h]}^{2}\right. \\
& +u)+\left(f_{3 R R}^{i} f_{3 L R}^{j}+f_{3 L L}^{i} f_{3 R L}^{j}\right) m_{t} m_{\tilde{\chi}_{i}^{0}}\left(m_{\tilde{\chi}}^{2}-m_{H[h]}^{2}+u\right)-\left(f_{3 R L}^{i} f_{3 L R}^{j}\right. \\
& \left.\left.+f_{3 L R}^{i} f_{3 R L}^{j}\right) 2 m_{t} m_{\tilde{\chi}} u-\left(f_{3 R R}^{i} f_{3 L L}^{j}+f_{3 L L}^{i} f_{3 R R}^{j}\right) 2 m_{\tilde{\chi}_{i}^{0}} m_{\tilde{\chi}_{j}^{0}} m_{t} m_{\tilde{\chi}}\right) \\
& /\left(\left(u-m_{\tilde{\chi}_{i}^{0}}^{2}\right)\left(u-m_{\tilde{\chi}_{j}^{0}}^{2}\right)\right) \\
& \mathcal{T}_{\mathrm{I}} \times \mathcal{T}_{\mathrm{II}}=(1 / 2)\left(\left(f_{1 L} f_{2 L}^{i}+f_{1 R} f_{2 R}^{i}\right) m_{t}\left(s+2 m_{\tilde{\chi}}^{2}+m_{t}^{2}-m_{\tilde{t}_{1}}^{2}-t\right)-\left(f_{1 R} f_{2 L}^{i}\right.\right. \\
& \left.\left.+f_{1 L} f_{2 R}^{i}\right) m_{\tilde{\chi}}\left(s+3 m_{t}^{2}-m_{H[h]}^{2}\right)\right) /\left(\left(s-m_{t}^{2}\right)\left(t-m_{\tilde{t}_{i}}^{2}\right)\right) \\
& \mathcal{T}_{\mathrm{I}} \times \mathcal{T}_{\text {III }}=(1 / 2)\left(( f _ { 1 L } f _ { 3 R L } ^ { i } + f _ { 1 R } f _ { 3 L R } ^ { i } ) \left(\left(s+m_{\tilde{\chi}}^{2}-m_{\tilde{t}_{1}}^{2}\right)\left(u+m_{t}^{2}-m_{\tilde{t}_{1}}^{2}\right)-\left(m_{\tilde{\chi}}^{2}+m_{t}^{2}\right.\right.\right. \\
& -t)\left(m_{t}^{2}+m_{\tilde{\chi}}^{2}-m_{\tilde{t}_{1}}^{2}-m_{H[h]}^{2}\right)+\left(u+m_{\tilde{\chi}}^{2}-m_{H[h]}^{2}\right)\left(s+m_{t}^{2}-m_{H[h]}^{2}\right) \\
& \left.+2 m_{t}\left(u+m_{\tilde{\chi}}^{2}-m_{H[h]}^{2}\right)\right)+\left(f_{1 L} f_{3 L L}^{i}+f_{1 R} f_{3 R R}^{i}\right) m_{\tilde{\chi}_{i}^{0}} m_{t}\left(s+2 m_{\tilde{\chi}}^{2}+m_{t}^{2}-m_{\tilde{t}_{1}}^{2}\right. \\
& -t)-\left(f_{1 L} f_{3 L R}^{i}+f_{1 R} f_{3 R L}^{i}\right) m_{\tilde{\chi}} m_{t}\left(u+2 m_{t}^{2}+m_{\tilde{\chi}}^{2}-2 m_{\tilde{t}_{1}}^{2}-m_{H[h]}^{2}\right) \\
& \left.-\left(f_{1 L} f_{3 R R}^{i}+f_{1 R} f_{3 L L}^{i}\right) m_{\tilde{\chi}} m_{\tilde{\chi}_{i}^{0}}\left(s+3 m_{t}^{2}-m_{H[h]}^{2}\right)\right) /\left(\left(s-m_{t}^{2}\right)\left(u-m_{\tilde{\chi}_{i}^{0}}^{2}\right)\right) \\
& \mathcal{T}_{\text {II }} \times \mathcal{T}_{\text {III }}=(1 / 2)\left(\left(f_{2 R}^{j} f_{3 L R}^{i}+f_{2 L}^{j} f_{3 R L}^{i}\right) m_{t}\left(u+m_{\tilde{\chi}}^{2}-m_{H[h]}^{2}\right)+\left(f_{2 L}^{j} f_{3 L L}^{i}+f_{2 R}^{j} f_{3 R R}^{i}\right)\right. \\
& \times m_{\tilde{\chi}_{i}^{0}}\left(m_{\tilde{\chi}}^{2}+m_{t}^{2}-t\right)-\left(f_{2 L}^{j} f_{3 L R}^{i}+f_{2 R}^{j} f_{3 R L}^{i}\right) m_{\tilde{\chi}}\left(u+m_{t}^{2}-m_{\tilde{t}_{1}}^{2}\right) \\
& \left.-\left(f_{2 R}^{j} f_{3 L L}^{i}+f_{2 L}^{j} f_{3 R R}^{i}\right) 2 m_{\tilde{\chi}} m_{t} m_{\tilde{\chi}_{i}^{0}}\right) /\left(\left(t-m_{\tilde{t}_{j}}^{2}\right)\left(u-m_{\tilde{\chi}_{i}^{0}}^{2}\right)\right)
\end{aligned}
$$




$$
\begin{aligned}
|\mathcal{T}|^{2}= & \mathcal{T}_{\mathrm{I}} \times \mathcal{T}_{\mathrm{I}}+\sum_{i, j=1}^{2} \mathcal{T}_{\text {II }} \times \mathcal{T}_{\text {II }}+\sum_{i, j=1}^{4} \mathcal{T}_{\text {III }} \times \mathcal{T}_{\text {III }}+2 \sum_{i=1}^{2} \mathcal{T}_{\text {I }} \times \mathcal{T}_{\text {II }}+2 \sum_{i=1}^{4} \mathcal{T}_{\mathrm{I}} \times \mathcal{T}_{\text {III }}+ \\
& 2 \sum_{j=1}^{2} \sum_{i=1}^{4} \mathcal{T}_{\text {II }} \times \mathcal{T}_{\text {III }}
\end{aligned}
$$

$\tilde{\chi} \tilde{t}_{1} \longrightarrow t A$

I. s-channel $t$ annihilation

II. t-channel $\tilde{t}_{2}$ exchange

III. u-channel $\chi_{(1,2,3,4)}^{0}$ exchange

$$
\begin{aligned}
& f_{1 L}=C_{\tilde{t}_{1}-t-\tilde{\chi}_{1}^{0}}^{L} C_{t-t-A} \\
& f_{1 R}=C_{\tilde{t}_{1}-t-\tilde{\chi}_{1}^{0}}^{R} C_{t-t-A} \\
& f_{2 L}=C_{\tilde{t}_{2}-t-\tilde{\chi}_{1}^{0}}^{L} C_{\tilde{t}_{1}-\tilde{t}_{2}-A} \\
& f_{2 R}=C_{\tilde{t}_{2}-t-\tilde{\chi}_{1}^{0}}^{R} C_{\tilde{t}_{1}-\tilde{t}_{2}-A} \\
& f_{3 L L}^{i}=C_{\tilde{t}_{1}-t-\tilde{\chi}_{i}^{0}}^{L} C_{\tilde{\chi}_{1}^{0}-\tilde{\chi}_{i}^{0}-A}^{L} \\
& f_{3 L R}^{i}=C_{\tilde{t}_{1}-t-\tilde{\chi}_{i}^{0}}^{L} C_{\tilde{\chi}_{1}^{0}-\tilde{\chi}_{i}^{0}-A}^{R} \\
& f_{3 R L}^{i}=C_{\tilde{t}_{1}-t-\tilde{\chi}_{i}^{0}}^{R} C_{\tilde{\chi}_{1}^{0}-\tilde{\chi}_{i}^{0}-A}^{L} \\
& f_{3 R R}^{i}=C_{\tilde{t}_{1}-t-\tilde{\chi}_{i}^{0}}^{R} C_{\tilde{\chi}_{1}^{0}-\tilde{\chi}_{i}^{0}-A}^{R} \\
& \mathcal{T}_{\mathrm{I}} \times \mathcal{T}_{\mathrm{I}}=(1 / 2)\left(\left(f_{1 L}^{2}+f_{1 R}^{2}\right)\left(\left(s-m_{t}^{2}-m_{A}^{2}\right)\left(s+m_{\tilde{\chi}}^{2}-m_{\tilde{t}_{1}}^{2}\right)-\left(s-m_{t}^{2}\right)\left(m_{\tilde{\chi}}^{2}+m_{t}^{2}-t\right)\right)\right. \\
& \left.-f_{1 L} f_{1 R} 4 m_{\tilde{\chi}} m_{t} m_{A}^{2}\right) /\left(s-m_{t}^{2}\right)^{2} \\
& \mathcal{T}_{\text {II }} \times \mathcal{T}_{\text {II }}=(1 / 2)\left(\left(f_{2 L} f_{2 L}+f_{2 R} f_{2 R}\right)\left(m_{\tilde{\chi}}^{2}+m_{t}^{2}-t\right)-2 m_{t} m_{\tilde{\chi}}\left(f_{2 L} f_{2 R}+f_{2 R} f_{2 L}\right)\right) \\
& /\left(\left(t-m_{\tilde{t}_{2}}^{2}\right)^{2}\right) \\
& \mathcal{T}_{\text {III }} \times \mathcal{T}_{\text {III }}=(1 / 2)\left(( f _ { 3 L R } ^ { i } f _ { 3 L R } ^ { j } + f _ { 3 R L } ^ { i } f _ { 3 R L } ^ { j } ) \left(\left(m_{t}^{2}-m_{\tilde{t}_{1}}^{2}+u\right)\left(m_{\tilde{\chi}}^{2}-m_{A}^{2}+u\right)-u\left(m_{\tilde{\chi}}^{2}+m_{t}^{2}\right.\right.\right. \\
& -t))+\left(f_{3 L L}^{i} f_{3 L L}^{j}+f_{3 R R}^{i} f_{3 R R}^{j}\right) m_{\tilde{\chi}_{i}^{0}} m_{\tilde{\chi}_{j}^{0}}\left(m_{\tilde{\chi}}^{2}+m_{t}^{2}-t\right)-\left(f_{3 L R}^{i} f_{3 L L}^{j}\right. \\
& \left.+f_{3 R L}^{i} f_{3 R R}^{j}\right) m_{\tilde{\chi}} m_{\tilde{\chi}_{j}^{0}}\left(m_{t}^{2}-m_{\tilde{t}_{1}}^{2}+u\right)-\left(f_{3 L L}^{i} f_{3 L R}^{j}+f_{3 R R}^{i} f_{3 R L}^{j}\right) m_{\tilde{\chi}_{i}^{0}} m_{\tilde{\chi}}\left(m_{t}^{2}\right. \\
& \left.-m_{\tilde{t}_{1}}^{2}+u\right)+\left(f_{3 R L}^{i} f_{3 L L}^{j}+f_{3 L R}^{i} f_{3 R R}^{j}\right) m_{t} m_{\tilde{\chi}_{j}^{0}}\left(m_{\tilde{\chi}}^{2}-m_{A}^{2}+u\right)+\left(f_{3 R R}^{i} f_{3 L R}^{j}\right. \\
& \left.+f_{3 L L}^{i} f_{3 R L}^{j}\right) m_{t} m_{\tilde{\chi}_{i}^{0}}\left(m_{\tilde{\chi}}^{2}-m_{A}^{2}+u\right)-\left(f_{3 R L}^{i} f_{3 L R}^{j}+f_{3 L R}^{i} f_{3 R L}^{j}\right) 2 m_{t} m_{\tilde{\chi}} u \\
& \left.-\left(f_{3 R R}^{i} f_{3 L L}^{j}+f_{3 L L}^{i} f_{3 R R}^{j}\right) 2 m_{\tilde{\chi}_{i}^{0}} m_{\tilde{\chi}_{j}^{0}} m_{t} m_{\tilde{\chi}}\right) /\left(\left(u-m_{\tilde{\chi}_{i}^{0}}^{2}\right)\left(u-m_{\tilde{\chi}_{j}^{0}}^{2}\right)\right) \\
& \mathcal{T}_{\mathrm{I}} \times \mathcal{T}_{\mathrm{II}}=(1 / 2)\left(\left(f_{2 R} f_{1 R}-f_{2 L} f_{1 L}\right) m_{t}\left(u-m_{\tilde{\chi}}^{2}-m_{A}^{2}\right)+\left(f_{2 L} f_{1 R}-f_{2 R} f_{1 L}\right) m_{\tilde{\chi}}\left(s-m_{t}^{2}\right.\right.
\end{aligned}
$$




$$
\begin{aligned}
& \left.\left.-m_{A}^{2}\right)\right) /\left(\left(s-m_{t}^{2}\right)\left(t-m_{\tilde{t}_{2}} 2\right)\right) \\
& \mathcal{T}_{\mathrm{I}} \times \mathcal{T}_{\text {III }}=(1 / 2)\left(( f _ { 3 L R } ^ { i } f _ { 1 R } - f _ { 3 R L } ^ { i } f _ { 1 L } ) \left(\left(m_{\tilde{\chi}}^{2}+m_{t}^{2}-t\right)\left(m_{t}^{2}+m_{\tilde{\chi}}^{2}-m_{\tilde{t}_{1}}^{2}-m_{A}^{2}\right)\right.\right. \\
& \left.-\left(s-m_{t}^{2}-m_{A}^{2}\right)\left(u+m_{\tilde{\chi}}^{2}-m_{A}^{2}\right)-\left(u+m_{t}^{2}-m_{\tilde{t}_{1}}^{2}\right)\left(s+m_{\tilde{\chi}}^{2}-m_{\tilde{t}_{1}}^{2}\right)\right) / 2 \\
& +\left(f_{3 R R}^{i} f_{1 R}-f_{3 L L}^{i} f_{1 L}\right) m_{\tilde{\chi}_{i}^{0}} m_{t}\left(u-m_{A}^{2}-m_{\tilde{\chi}}^{2}\right)+\left(f_{3 L R}^{i} f_{1 L}-f_{3 R L}^{i} f_{1 R}\right) \\
& \left.\times m_{t} m_{\tilde{\chi}}\left(u-m_{\tilde{\chi}}^{2}+m_{A}^{2}\right)+\left(f_{3 R R}^{i} f_{1 L}-f_{3 L L}^{i} f_{1 R}\right) m_{\tilde{\chi}_{i}^{0}} m_{\tilde{\chi}}\left(m_{t}^{2}+m_{A}^{2}-s\right)\right) \\
& /\left(\left(s-m_{t}^{2}\right)\left(u-m_{\tilde{\chi}_{i}^{0}}^{2}\right)\right) \\
& \mathcal{T}_{\text {II }} \times \mathcal{T}_{\text {III }}=(1 / 2)\left(\left(f_{2 R} f_{3 L R}^{i}+f_{2 L} f_{3 R L}^{i}\right) m_{t}\left(u+m_{\tilde{\chi}}^{2}-m_{A}^{2}\right)+\left(f_{2 L} f_{3 L L}^{i}+f_{2 R} f_{3 R R}^{i}\right)\right. \\
& \times m_{\tilde{\chi}_{i}^{0}}\left(m_{\tilde{\chi}}^{2}+m_{t}^{2}-t\right)-\left(f_{2 L} f_{3 L R}^{i}+f_{2 R} f_{3 R L}^{i}\right) m_{\tilde{\chi}}\left(u+m_{t}^{2}-m_{\tilde{t}_{1}}^{2}\right) \\
& \left.-\left(f_{2 R} f_{3 L L}^{i}+f_{2 L} f_{3 R R}^{i}\right) 2 m_{\tilde{\chi}} m_{t} m_{\tilde{\chi}_{i}^{0}}\right) /\left(\left(t-m_{\tilde{t}_{2}}^{2}\right)\left(u-m_{\tilde{\chi}_{i}^{0}}^{2}\right)\right) \\
& |\mathcal{T}|^{2}=\mathcal{T}_{\mathrm{I}} \times \mathcal{T}_{\mathrm{I}}+\mathcal{T}_{\text {II }} \times \mathcal{T}_{\text {II }}+\sum_{i, j=1}^{4} \mathcal{T}_{\text {III }} \times \mathcal{T}_{\text {III }}+2 \mathcal{T}_{\mathrm{I}} \times \mathcal{T}_{\text {II }}+2 \sum_{i=1}^{4} \mathcal{T}_{\mathrm{I}} \times \mathcal{T}_{\text {III }}+2 \sum_{i=1}^{4} \mathcal{T}_{\text {II }} \times \mathcal{T}_{\text {III }}
\end{aligned}
$$

$\tilde{\chi} \tilde{t}_{1} \longrightarrow b H^{+}$

I. s-channel $t$ annihilation

II. t-channel $\tilde{\chi}_{(1,2)}^{+}$exchange

III. u-channel $\tilde{b}_{(1,2)}$ exchange

$$
\begin{aligned}
f_{1 L L} & =C_{t-b-H^{+}}^{L} C_{\tilde{t}_{1}-t-\tilde{\chi}_{1}^{0}}^{L} \\
f_{1 L R} & =C_{t-b-H^{+}}^{L} C_{\tilde{t}_{1}-t-\tilde{\chi}_{1}^{0}}^{R} \\
f_{1 R L} & =C_{t-b-H^{+}}^{R} C_{\tilde{t}_{1}-t-\tilde{\chi}_{1}^{0}}^{L} \\
f_{1 R R} & =C_{t-b-H^{+}}^{R} C_{\tilde{t}_{1}-t-\tilde{\chi}_{1}^{0}}^{R} \\
f_{2 L L}^{i} & =C_{\tilde{t}_{1}-b-\tilde{\chi}_{i}^{+}}^{L} C_{\tilde{\chi}_{1}^{0}-\tilde{\chi}_{i}^{+}-H^{+}}^{L} \\
f_{2 L R}^{i} & =C_{\tilde{t}_{1}-b-\tilde{\chi}_{i}^{+}}^{L} C_{\tilde{\chi}_{1}^{0}-\tilde{\chi}_{i}^{+}-H^{+}}^{R} \\
f_{2 R L}^{i} & =C_{\tilde{t}_{1}-b-\tilde{\chi}_{i}^{+}}^{R} C_{\tilde{\chi}_{1}^{0}-\tilde{\chi}_{i}^{+}-H^{+}}^{L} \\
f_{2 R R}^{i} & =C_{\tilde{t}_{1}-b-\tilde{\chi}_{i}^{+}}^{R} C_{\tilde{\chi}_{1}^{0}-\tilde{\chi}_{i}^{+}-H^{+}}^{R} \\
f_{3 L}^{i} & =C_{\tilde{b}_{i}-b-\tilde{\chi}_{1}^{0}}^{L} C_{\tilde{t}_{1}-\tilde{b}_{i}-H^{+}} \\
f_{3 R}^{i} & =C_{\tilde{b}_{i}-b-\tilde{\chi}_{1}^{0}}^{R} C_{\tilde{t}_{1}-\tilde{b}_{i}-H^{+}} \\
\mathcal{T}_{\mathrm{I}} \times \mathcal{T}_{\mathrm{I}} & =(1 / 2)\left(\left(f_{1 L R}^{2}+f_{1 R L}^{2}\right)\left(\left(s+m_{b}^{2}-m_{H^{+}}^{2}\right)\left(s+m_{\tilde{\chi}}^{2}-m_{\tilde{t}_{1}}^{2}\right)-s\left(m_{\tilde{\chi}}^{2}+m_{b}^{2}-u\right)\right)\right.
\end{aligned}
$$


$+\left(f_{1 L L}^{2}+f_{1 R R}^{2}\right) m_{t}^{2}\left(m_{\tilde{\chi}}^{2}+m_{b}^{2}-u\right)-2\left(f_{1 L L} f_{1 L R}+f_{1 R R} f_{1 R L}\right) m_{\tilde{\chi}} m_{t}$

$\times\left(s+m_{b}^{2}-m_{H^{+}}^{2}\right)+2\left(f_{1 L R} f_{1 R R}+f_{1 L L} f_{1 R L}\right) m_{b} m_{t}\left(s+m_{\tilde{\chi}}^{2}-m_{\tilde{t}_{1}}^{2}\right)$

$\left.-4\left(f_{1 L R} f_{1 R L}\right) m_{b} m_{\tilde{\chi}} s-4 f_{1 L L} f_{1 R R} m_{t}^{2} m_{\tilde{\chi}} m_{b}\right) /\left(s-m_{t}^{2}\right)^{2}$

$$
\begin{aligned}
\mathcal{T}_{\text {II }} \times \mathcal{T}_{\text {II }}= & (1 / 2)\left(( f _ { 2 R R } ^ { i } f _ { 2 R R } ^ { j } + f _ { 2 L L } ^ { i } f _ { 2 L L } ^ { j } ) \left(\left(t+m_{b}^{2}-m_{\tilde{t}_{1}}^{2}\right)\left(t+m_{\tilde{\chi}}^{2}-m_{H^{+}}^{2}\right)-t\left(m_{\tilde{\chi}}^{2}+m_{b}^{2}\right.\right.\right. \\
& -u))+\left(f_{2 R L}^{i} f_{2 R L}^{j}+f_{2 L R}^{i} f_{2 L R}^{j}\right) m_{\tilde{\chi}_{i}^{+}} m_{\tilde{\chi}_{j}^{+}}\left(m_{\tilde{\chi}}^{2}+m_{b}^{2}-u\right)-\left(f_{2 R R}^{i} f_{2 R L}^{j}\right. \\
& \left.+f_{2 L L}^{i} f_{2 L R}^{j}\right) m_{\tilde{\chi}} m_{\tilde{\chi}_{j}^{+}}\left(t+m_{b}^{2}-m_{\tilde{t}_{1}}^{2}\right)-\left(f_{2 R L}^{i} f_{2 R R}^{j}+f_{2 L R}^{i} f_{2 L L}^{j}\right) m_{\tilde{\chi}_{i}^{+}} m_{\tilde{\chi}} \\
& \times\left(t-m_{b}^{2}-m_{\tilde{t}_{1}}^{2}\right)+\left(f_{2 L L}^{i} f_{2 R L}^{j}+f_{2 R R}^{i} f_{2 L R}^{j}\right) m_{b} m_{\tilde{\chi}_{j}^{+}}\left(t+m_{\tilde{\chi}}^{2}-m_{H^{+}}^{2}\right) \\
& +\left(f_{2 L R}^{i} f_{2 R R}^{j}+f_{2 R L}^{i} f_{2 L L}^{j}\right) m_{b} m_{\tilde{\chi}_{i}^{+}}\left(t+m_{\tilde{\chi}}^{2}-m_{H^{+}}^{2}\right)-\left(f_{2 L L}^{i} f_{2 R R}^{j}+f_{2 R R}^{i} f_{2 L L}^{j}\right) \\
& \left.\times 2 m_{b} m_{\tilde{\chi}} t-\left(f_{2 L R}^{i} f_{2 R L}^{j}+f_{2 R L}^{i} f_{2 L R}^{j}\right) 2 m_{b} m_{\tilde{\chi}} m_{\tilde{\chi}_{i}^{+}} m_{\tilde{\chi}_{j}^{+}}\right) \\
& /\left(\left(t-m_{\tilde{\chi}_{i}^{+}}^{2}\right)\left(t-m_{\tilde{\chi}_{j}^{+}}^{2}\right)\right) \\
\mathcal{T}_{\text {III }} \times \mathcal{T}_{\text {III }}= & (1 / 2)\left(\left(f_{3 L}^{i} f_{3 L}^{j}+f_{3 R}^{i} f_{3 R}^{j}\right)\left(m_{\tilde{\chi}}^{2}+m_{b}^{2}-u\right)-2 m_{\tilde{\chi}} m_{b}\left(f_{3 L}^{i} f_{3 R}^{j}+f_{3 R}^{i} f_{3 L}^{j}\right)\right) \\
& /\left(\left(u-m_{\tilde{b}_{i}}^{2}\right)\left(u-m_{\tilde{b}_{j}}^{2}\right)\right)
\end{aligned}
$$

$$
\begin{aligned}
\mathcal{T}_{\mathrm{I}} \times \mathcal{T}_{\mathrm{II}}= & (1 / 2)\left(( f _ { 2 R R } ^ { i } f _ { 1 L R } + f _ { 2 L L } ^ { i } f _ { 1 R L } ) ( 1 / 2 ) \left(\left(t+m_{b}^{2}-m_{\tilde{t}_{1}}^{2}\right)\left(s+m_{\tilde{\chi}}^{2}-m_{\tilde{t}_{1}}^{2}\right)-\left(m_{\tilde{\chi}}^{2}\right.\right.\right. \\
& \left.\left.+m_{b}^{2}-u\right)\left(m_{\tilde{\chi}}^{2}+m_{b}^{2}-m_{\tilde{t}_{1}}^{2}-m_{H^{+}}^{2}\right)+\left(s+m_{b}^{2}-m_{H^{+}}^{2}\right)\left(t+m_{\tilde{\chi}}^{2}-m_{H^{+}}^{2}\right)\right) \\
& +\left(f_{2 R L}^{i} f_{1 L L}+f_{2 L R}^{i} f_{1 R R}\right) m_{\tilde{\chi}_{i}^{+}} m_{t}\left(m_{\tilde{\chi}}^{2}+m_{b}^{2}-u\right)-\left(f_{2 R R}^{i} f_{1 L L}+f_{2 L L}^{i} f_{1 R R}\right) \\
& \times m_{\tilde{\chi}} m_{t}\left(t+m_{b}^{2}-m_{\tilde{t}_{1}}^{2}\right)-\left(f_{2 R L}^{i} f_{1 L R}+f_{2 L R}^{i} f_{1 R L}\right) m_{\tilde{\chi}_{i}^{+}} m_{\tilde{\chi}}\left(s+m_{b}^{2}-m_{H^{+}}^{2}\right) \\
& +\left(f_{2 L L}^{i} f_{1 L L}+f_{2 R R}^{i} f_{1 R R}\right) m_{b} m_{t}\left(t+m_{\tilde{\chi}}^{2}-m_{H^{+}}^{2}\right)+\left(f_{2 L R}^{i} f_{1 L R}+f_{2 R L}^{i} f_{1 R L}\right) \\
& \times m_{b} m_{\tilde{\chi}_{i}^{+}}\left(s+m_{\tilde{\chi}}^{2}-m_{\tilde{t}_{1}}^{2}\right)-\left(f_{2 L L}^{i} f_{1 L R}+f_{2 R R}^{i} f_{1 R L}\right) m_{b} m_{\tilde{\chi}}\left(m_{\tilde{\chi}}^{2}+m_{b}^{2}-m_{\tilde{t}_{1}}^{2}\right. \\
& \left.\left.-m_{H^{+}}^{2}\right)-\left(f_{2 L R}^{i} f_{1 L L}+f_{2 R L}^{i} f_{1 R R}\right) 2 m_{b} m_{\tilde{\chi}} m_{t} m_{\tilde{\chi}_{i}^{+}}\right) /\left(\left(s-m_{t}^{2}\right)\left(t-m_{\tilde{\chi}_{i}^{+}}^{2}\right)\right) \\
\mathcal{T}_{\mathrm{I}} \times \mathcal{T}_{\mathrm{III}}= & (1 / 2)\left(\left(f_{1 L R} f_{3 R}^{i}+f_{1 R L} f_{3 L}^{i}\right) m_{b}\left(s+m_{\tilde{\chi}}^{2}-m_{\tilde{t}_{1}}^{2}\right)+\left(f_{1 L L} f_{3 L}^{i}+f_{1 R R} f_{3 R}^{i}\right) m_{t}\right. \\
& \times\left(m_{\tilde{\chi}}^{2}+m_{b}^{2}-u\right)-\left(f_{1 L R} f_{3 L}^{i}+f_{1 R L} f_{3 R}^{i}\right) m_{\tilde{\chi}}\left(s+m_{b}^{2}-m_{H^{+}}^{2}\right)-\left(f_{1 L L} f_{3 R}^{i}\right. \\
& \left.\left.+f_{1 R R} f_{3 L}^{i}\right) 2 m_{t} m_{b} m_{\tilde{\chi}}\right) /\left(\left(u-m_{\tilde{b}_{i}}^{2}\right)\left(s-m_{t}^{2}\right)\right) \\
\mathcal{T}_{\mathrm{II}} \times \mathcal{T}_{\mathrm{III}}= & (1 / 2)\left(\left(f_{2 L R}^{j} f_{3 R}^{i}+f_{2 R L}^{j} f_{3 L}^{i}\right) m_{b}\left(t+m_{\tilde{\chi}}^{2}-m_{H^{+}}^{2}\right)+\left(f_{2 L L}^{j} f_{3 L}^{i}+f_{2 R R}^{j} f_{3 R}^{i}\right)\right. \\
& \times m_{\tilde{\chi}_{j}^{+}}\left(m_{\tilde{\chi}}^{2}+m_{b}^{2}-u\right)-\left(f_{2 L R}^{j} f_{3 L}^{i}+f_{2 R L}^{j} f_{3 R}^{i}\right) m_{\tilde{\chi}}\left(t+m_{b}^{2}-m_{\tilde{t}_{1}}^{2}\right) \\
& \left.-\left(f_{2 L L}^{j} f_{3 R}^{i}+f_{2 R R}^{j} f_{3 L}^{i}\right) 2 m_{\tilde{\chi}_{j}^{+}} m_{b} m_{\tilde{\chi}}\right) /\left(\left(u-m_{\tilde{b}_{i}}^{2}\right)\left(t-m_{\tilde{\chi}_{j}^{+}}^{2}\right)\right) \\
= & \mathcal{T}_{\mathrm{I}} \times \mathcal{T}_{\mathrm{I}}+\sum_{i, j=1}^{2} \mathcal{T}_{\mathrm{II}} \times \mathcal{T}_{\mathrm{II}}+\sum_{i, j=1}^{2} \mathcal{T}_{\mathrm{III}} \times \mathcal{T}_{\mathrm{III}}+2 \sum_{i=1}^{2} \mathcal{T}_{\mathrm{I}} \times \mathcal{T}_{\mathrm{II}}+2 \sum_{i=1}^{2} \mathcal{T}_{\mathrm{I}} \times \mathcal{T}_{\mathrm{III}}+ \\
|\mathcal{T}|^{2} &
\end{aligned}
$$




$$
2 \sum_{i, j=1}^{2} \mathcal{T}_{\text {II }} \times \mathcal{T}_{\text {III }}
$$

$\tilde{t}_{1} \tilde{\ell} \longrightarrow t \ell$

I. t-channel $\tilde{\chi}_{(1,2,3,4)}^{0}$ exchange

$$
\begin{aligned}
f_{L L}^{i}= & C_{\tilde{t}_{1}-t-\tilde{\chi}_{i}^{0}}^{L} C_{\tilde{\ell}_{1}-\ell-\tilde{\chi}_{i}^{0}}^{L} \\
f_{L R}^{i}= & C_{\tilde{t}_{1}-t-\tilde{\chi}_{i}^{0}}^{L} C_{\tilde{\ell}_{1}-\ell-\tilde{\chi}_{i}^{0}}^{R} \\
f_{R L}^{i}= & C_{\tilde{t}_{1}-t-\tilde{\chi}_{i}^{0}}^{R} C_{\tilde{\ell}_{1}-\ell-\tilde{\chi}_{i}^{0}}^{L} \\
f_{R R}^{i}= & C_{\tilde{t}_{1}-t-\tilde{\chi}_{i}^{0}}^{R} C_{\tilde{\ell}_{1}-\ell-\tilde{\chi}_{i}^{0}}^{R} \\
\mathcal{T}_{\mathrm{I}} \times \mathcal{T}_{\mathrm{I}}= & \left(\left(f_{L R}^{i} f_{R L}^{j}+f_{R L}^{i} f_{L R}^{j}\right)\left(\left(m_{\tilde{t}_{1}}^{2}-m_{t}^{2}-t\right)\left(t+m_{\ell}^{2}-m_{\tilde{\ell}_{1}}^{2}\right)-t\left(s-m_{t}^{2}-m_{\ell}^{2}\right)\right)\right. \\
& +\left(f_{L L}^{i} f_{L L}^{j}+f_{R R}^{i} f_{R R}^{j}\right) m_{\tilde{\chi}_{i}^{0}} m_{\tilde{\chi}_{j}^{0}}\left(s-m_{t}^{2}-m_{\ell}^{2}\right) \\
& -\left(f_{L R}^{i} f_{L L}^{j}+f_{R L}^{i} f_{R R}^{j}\right) m_{\ell} m_{\tilde{\chi}_{j}^{0}}\left(m_{\tilde{t}_{1}}^{2}-m_{t}^{2}-t\right) \\
& -\left(f_{L L}^{i} f_{R L}^{j}+f_{R R}^{i} f_{L R}^{j}\right) m_{\ell} m_{\tilde{\chi}_{i}^{0}}\left(m_{\tilde{t}_{1}}^{2}-m_{t}^{2}-t\right) \\
& +\left(f_{R L}^{i} f_{L L}^{j}+f_{L R}^{i} f_{R R}^{j}\right) m_{t} m_{\tilde{\chi}_{j}^{0}}\left(t+m_{\ell}^{2}-m_{\tilde{\ell}_{1}}^{2}\right) \\
& +\left(f_{R R}^{i} f_{R L}^{j}+f_{L L}^{i} f_{L R}^{j}\right) m_{t} m_{\tilde{\chi}_{i}^{0}}\left(t+m_{\ell}^{2}-m_{\tilde{\ell}_{1}}^{2}\right) \\
& \left.-\left(f_{R L}^{i} f_{L R}^{j}+f_{L R}^{i} f_{L R}^{j}\right) m_{t} m_{\ell} 2 t-\left(f_{R R}^{i} f_{L L}^{j}+f_{L L}^{i} f_{R R}^{j}\right) 2 m_{t} m_{\ell} m_{\tilde{\chi}_{i}^{0}} m_{\tilde{\chi}_{j}^{0}}\right) \\
& /\left(\left(t-m_{\tilde{\chi}_{i}^{0}}^{2}\right)\left(t-m_{\tilde{\chi}_{j}^{0}}^{2}\right)\right) \\
|\mathcal{T}|^{2}= & \sum_{i, j=1}^{4} \mathcal{T}_{\mathrm{I}} \times \mathcal{T}_{\mathrm{I}}
\end{aligned}
$$

$\tilde{t}_{1} \tilde{\ell} \longrightarrow b \nu$

I. t-channel $\tilde{\chi}_{(1,2)}^{+}$exchange

$$
\begin{aligned}
& f_{L L}^{i}=C_{\tilde{t}_{1}-b-\tilde{\chi}_{i}^{+}}^{L} C_{\tilde{\ell}_{1}-\nu-\tilde{\chi}_{i}^{+}}^{L} \\
& f_{L R}^{i}=C_{\tilde{t}_{1}-b-\tilde{\chi}_{i}^{+}}^{L} C_{\tilde{\ell}_{1}-\nu-\tilde{\chi}_{i}^{+}}^{R} \\
& f_{R L}^{i}=C_{\tilde{t}_{1}-b-\tilde{\chi}_{i}^{+}}^{R} C_{\tilde{\ell}_{1}-\nu-\tilde{\chi}_{i}^{+}}^{L} \\
& f_{R R}^{i}=C_{\tilde{t}_{1}-b-\tilde{\chi}_{i}^{+}}^{R} C_{\tilde{\ell}_{1}-\nu-\tilde{\chi}_{i}^{+}}^{R}
\end{aligned}
$$




$$
\begin{aligned}
\mathcal{T}_{\mathrm{I}} \times \mathcal{T}_{\mathrm{I}}= & \left(\left(f_{L R}^{i} f_{R L}^{j}+f_{R L}^{i} f_{L R}^{j}\right)\left(\left(m_{\tilde{t}_{1}}^{2}-m_{b}^{2}-t\right)\left(t-m_{\tilde{\ell}_{1}}^{2}\right)-t\left(s-m_{b}^{2}\right)\right)\right. \\
& +\left(f_{L L}^{i} f_{L L}^{j}+f_{R R}^{i} f_{R R}^{j}\right) m_{\tilde{\chi}_{i}^{+}} m_{\tilde{\chi}_{j}^{+}}\left(s-m_{b}^{2}\right) \\
& +\left(f_{R L}^{i} f_{L L}^{j}+f_{L R}^{i} f_{R R}^{j}\right) m_{b} m_{\tilde{\chi}_{j}^{+}}\left(t-m_{\tilde{\ell}_{1}}^{2}\right) \\
& \left.+\left(f_{R R}^{i} f_{R L}^{j}+f_{L L}^{i} f_{L R}^{j}\right) m_{b} m_{\tilde{\chi}_{i}^{+}}\left(t-m_{\tilde{\ell}_{1}}^{2}\right)\right) \\
& /\left(\left(t-m_{\tilde{\chi}_{i}^{+}}^{2}\right)\left(t-m_{\tilde{\chi}_{j}^{+}}^{2}\right)\right) \\
|\mathcal{T}|^{2}= & \sum_{i, j=1}^{2} \mathcal{T}_{\mathrm{I}} \times \mathcal{T}_{\mathrm{I}}
\end{aligned}
$$

$\tilde{t}_{1} \tilde{\ell}^{*} \longrightarrow t \bar{\ell}$

I. t-channel $\tilde{\chi}_{(1,2,3,4)}^{0}$ exchange

$$
\begin{aligned}
f_{L L}^{i}= & C_{\tilde{t}_{1}-t-\tilde{\chi}_{i}^{0}}^{L} C_{\tilde{\ell}_{1}-\ell-\tilde{\chi}_{i}^{0}}^{L} \\
f_{L R}^{i}= & C_{\tilde{t}_{1}-t-\tilde{\chi}_{i}^{0}}^{L} C_{\tilde{\ell}_{1}-\ell-\tilde{\chi}_{i}^{0}}^{R} \\
f_{R L}^{i}= & C_{\tilde{t}_{1}-t-\tilde{\chi}_{i}^{0}}^{R} C_{\tilde{\ell}_{1}-\ell-\tilde{\chi}_{i}^{0}}^{L} \\
f_{R R}^{i}= & C_{\tilde{t}_{1}-t-\tilde{\chi}_{i}^{0}}^{R} C_{\tilde{\ell}_{1}-\ell-\tilde{\chi}_{i}^{0}}^{R} \\
\mathcal{T}_{\mathrm{I}} \times \mathcal{T}_{\mathrm{I}}= & \left(\left(f_{L L}^{i} f_{L L}^{j}+f_{R R}^{i} f_{R R}^{j}\right)\left(\left(m_{\tilde{t}_{1}}^{2}-m_{t}^{2}-t\right)\left(t+m_{\ell}^{2}-m_{\tilde{\ell}_{1}}^{2}\right)-t\left(s-m_{t}^{2}-m_{\ell}^{2}\right)\right)\right. \\
& +\left(f_{L R}^{i} f_{R L}^{j}+f_{R L}^{i} f_{L R}^{j}\right) m_{\tilde{\chi}_{i}^{0}} m_{\tilde{\chi}_{j}^{0}}\left(s-m_{t}^{2}-m_{\ell}^{2}\right) \\
& -\left(f_{L L}^{i} f_{R L}^{j}+f_{R R}^{i} f_{L R}^{j}\right) m_{\ell} m_{\tilde{\chi}_{j}^{0}}\left(m_{\tilde{t}_{1}}^{2}-m_{t}^{2}-t\right) \\
& -\left(f_{L R}^{i} f_{L L}^{j}+f_{R L}^{i} f_{R R}^{j}\right) m_{\ell} m_{\tilde{\chi}_{i}^{0}}\left(m_{\tilde{t}_{1}}^{2}-m_{t}^{2}-t\right) \\
& +\left(f_{R R}^{i} f_{R L}^{j}+f_{L L}^{i} f_{L R}^{j}\right) m_{t} m_{\tilde{\chi}_{j}^{0}}\left(t+m_{\ell}^{2}-m_{\tilde{\ell}_{1}}^{2}\right) \\
& +\left(f_{R L}^{i} f_{L L}^{j}+f_{L R}^{i} f_{R R}^{j}\right) m_{t} m_{\tilde{\chi}_{i}^{0}}\left(t+m_{\ell}^{2}-m_{\tilde{\ell}_{1}}^{2}\right) \\
& \left.-\left(f_{R R}^{i} f_{L L}^{j}+f_{L L}^{i} f_{R R}^{j}\right) m_{t} m_{\ell} 2 t-\left(f_{R L}^{i} f_{R L}^{j}+f_{L R}^{i} f_{L R}^{j}\right) 2 m_{t} m_{\ell} m_{\tilde{\chi}_{i}^{0}} m_{\tilde{\chi}_{j}^{0}}\right) \\
& /\left(\left(t-m_{\tilde{\chi}_{i}^{0}}^{2}\right)\left(t-m_{\tilde{\chi}_{j}^{0}}^{2}\right)\right) \\
|\mathcal{T}|^{2}= & \sum_{i, j=1}^{4} \mathcal{T}_{\mathrm{I}} \times \mathcal{T}_{\mathrm{I}}
\end{aligned}
$$

\section{References}


[1] J. Ellis, J.S. Hagelin, D.V. Nanopoulos, K.A. Olive and M. Srednicki, Nucl. Phys. B 238, 453 (1984); see also H. Goldberg, Phys. Rev. Lett. 50, 1419 (1983).

[2] C. Boehm, A. Djouadi and M. Drees, Phys. Rev. D 62, 035012 (2000) arXiv:hepph/9911496].

[3] N. Bahcall, J. P. Ostriker, S. Perlmutter and P. J. Steinhardt, Science 284, 1481 (1999).

[4] K. Griest and D. Seckel, Phys. Rev. D 43, 3191 (1991).

[5] S. Mizuta and M. Yamaguchi, Phys. Lett. B 298, 120 (1993) arXiv:hep-ph/9208251.

[6] J. Edsjo and P. Gondolo, Phys. Rev. D 56, 1879 (1997) arXiv:hep-ph/9704361.

[7] J. Ellis, T. Falk and K.A. Olive, Phys. Lett. B 444, 367 (1998).

[8] J. Ellis, T. Falk, K.A. Olive and M. Srednicki, Astropart. Phys. 13181 (2000).

[9] M. E. Gómez, G. Lazarides and C. Pallis, Phys. Rev. D 61, 123512 (2000) arXiv:hepph/9907261 and Phys. Lett. B 487, 313 (2000) arXiv:hep-ph/0004028; see also: A. B. Lahanas, D. V. Nanopoulos and V. C. Spanos, Phys. Rev. D 62, 023515 (2000), [arXive:hep-ph/9909497].

[10] R. Arnowitt, B. Dutta and Y. Santoso, Nucl. Phys. B 606, 59 (2001) arXiv:hepph/0102181.

[11] J. L. Feng, K. T. Matchev and T. Moroi, Phys. Rev. Lett. 84, 2322 (2000) arXiv:hepph/9908309]; J. L. Feng, K. T. Matchev and T. Moroi, Phys. Rev. D 61, 075005 (2000) arXiv:hep-ph/9909334; J. L. Feng, K. T. Matchev and F. Wilczek, Phys. Lett. B 482, 388 (2000) arXiv:hep-ph/0004043.

[12] J. R. Ellis, T. Falk, G. Ganis, K. A. Olive and M. Srednicki, Phys. Lett. B 510, 236 (2001) arXiv:hep-ph/0102098.

[13] M. Drees and M. M. Nojiri, Phys. Rev. D 47, 376 (1993); H. Baer and M. Brhlik, Phys. Rev. D 53, 597 (1996) and Phys. Rev. D 57, 567 (1998); H. Baer, M. Brhlik, M. A. Diaz, J. Ferrandis, P. Mercadante, P. Quintana and X. Tata, Phys. Rev. D 63, 015007 (2001); A. B. Lahanas and V. C. Spanos, hep-ph/0106345. 
[14] M. Srednicki, R. Watkins and K. A. Olive, Nucl. Phys. B 310, 693 (1988).

[15] P. Gondolo and G. Gelmini, Nucl. Phys. B 360, 145 (1991).

[16] T. Falk, K. A. Olive and M. Srednicki, Phys. Lett. B 339, 248 (1994) arXiv:hepph/9409270].

[17] H. N. Brown et al. [Muon g-2 Collaboration], Phys. Rev. Lett. 86, 2227 (2001) hepex/0102017].

[18] S. Heinemeyer, W. Hollik and G. Weiglein, Comput. Phys. Commun. 124, 76 (2000) arXiv:hep-ph/9812320; S. Heinemeyer, W. Hollik and G. Weiglein, Eur. Phys. J. C 9, 343 (1999) arXiv:hep-ph/9812472.

[19] H. Baer, M. Brhlik and D. Castaño, Phys. Rev. D 54 (1996) 6944; S. Abel and T. Falk, Phys. Lett. B 444 (1998) 427. 